\title{
Multirate Digital Filters, Filter Banks, Polyphase Networks, and Applications: A Tutorial
}

\author{
P. P. VAIDYANATHAN, SENIOR MEMBER, IEEE
}

\begin{abstract}
Multirate digital filters and filter banks find application in communications, speech processing, image compression, antenna systems, analog voice privacy systems, and in the digital audio industry. During the last several years there has been substantial progress in multirate system research. This includes design of decimation and interpolation filters, analysis/synthesis filter banks (also called quadrature mirror filters, or QMF), and the development of new sampling theorems. First, the basic concepts and building blocks in multirate digital signal processing (DSP), including the digital polyphase representation, are reviewed. Next, recent progress as reported by several authors in this area is discussed. Several applications are described, including the following: subband coding of waveforms, voice privacy systems, integral and fractional sampling rate conversion (such as in digital audio), digital crossover networks, and multirate coding of narrow-band filter coefficients. The M-band QMF bank is discussed in considerable detail, including an analysis of various errors and imperfections. Recent techniques for perfect signal reconstruction in such systems are reviewed. The connection between QMF banks and other related topics, such as block digital filtering and periodically time-varying systems, based on a pseudo-circulant matrix framework, is covered. Unconventional applications of the polyphase concept are discussed.
\end{abstract}

\section{INTRODUCTION}

In recent years there has been tremendous progress in the multirate processing of digital signals. Unlike the single-rate system, the sample spacing in a multirate system can vary from point to point [1], [2]. This often results in more efficient processing of signals because the sampling rates at various internal points can be kept as small as possible. Unfortunately, this also results in the introduction of a new type of error, i.e., aliasing, which should somehow be canceled eventually.

The basic building blocks in a multirate digital signal processing (DSP) system are decimators and interpolators. In 1981, an excellent tutorial article on decimation and interpolation appeared in [3]. Subsequent to this a text on the subject of multirate systems has also been published by the same authors [4]. Since then, a number of new developments have taken place in the area, particularly in multirate

Manuscript received October 12, 1988; revised June 13, 1989. This work was supported in part by the National Science Foundation under grants DCl 8552579 and MIP 8604456.

The author is with the Department of Electrical Engineering, California Institute of Technology, Pasadena, CA 91125, USA.

IEEE Log Number 8933329. digital filter bank designs. A short summary of some of these developments was reported recently by this author at an IEEE international conference [5]. The purpose of this article is to provide a self-contained and more complete exposure to many recent contributions on multirate systems, including filter bank design.

As mentioned in [3], multirate systems find application in communications, speech processing, spectrum analysis [6], radar systems, and antenna systems. In this tutorial, two sections are devoted to a review of applications. In Section III, we point out applications in digital audio systems, in subband coding techniques (used in speech and image compression), and in analog voice privacy systems (for standard telephone communications). In Section V-E, applications of special transfer functions (such as complementary functions) in digital audio is reviewed. In Section IX, several unconventional applications of multirate systems and polyphase theory are indicated. These include a) derivation of new sampling theorems for efficient compression of signals, b) derivation of new techniques for efficient coding of impulse response sequences of narrow band filters, c) design of FIR filters with adjustable multilevel responses, and d) adaptive filtering in subbands.

\section{A. Paper Outline}

In section II, basic tools, such as decimators, interpolators, decimation and interpolation filters, and digital filter banks, are reviewed, along with the interconnection properties of the building blocks. In section III, some applications of multirate DSP are indicated, in digital audio systems, in subband coding, and in voice privacy systems. Section IV reviews the digital polyphase decomposition due to Bellanger, along with applications such as the uniform DFT filter bank. The concept of multilevel polyphase decomposition is also introduced here as a tool for efficient implementation of fractional decimation filters. Several special types of filter banks, such as Nyquist filters, powercomplementary systems and Euclidean filter-banks, are studied in section V. In section VI, the two-band QMF bank is studied in sufficient detail along with procedures for eliminating aliasing in such systems. Procedures for elimination of amplitude and/or phase distortion are discussed. 
Perfect-reconstruction two-channel QMF banks are introduced by blending the polyphase concept with the classical network-theoretic concept of losslessness.

The relation between $M$-band QMF banks and two other related topics (block filtering and periodically time-varying systems) is reviewed in section VII, based on an algebraic structure called the pseudo-circulant matrix. In section VIII, $M$-band QMF banks are discussed in greater detail, and techniques for elimination of aliasing, amplitude, and phase distortions are reviewed. Section IX discusses unconventional applications, and Section $X$ discusses some extensions of multirate ideas to cases of multidimensional signals. The paper concludes with a discussion of open problems in multirate DSP.

\section{B. Notations and Terminology}

The variables $\Omega$ and $\omega$ are used as frequency variables for continuous-time and discrete-time cases, respectively. In the discrete-time case the term normalized frequency is used to denote $f=\omega / 2 \pi$. The frequency response of a transfer function $H(z)$ is expressed as $H\left(e^{j \omega}\right)=\left|H\left(e^{j \omega}\right)\right| e^{j \phi(\omega)}$, where $\left|H\left(e^{j \omega}\right)\right|$ is the magnitude response and $\phi(\omega)$ the phase response. The quantity $\tau(\omega)=-d \phi(\omega) / d \omega$ is the group delay of $H(z)$. If $\left|H\left(e^{j \omega}\right)\right|$ is constant for all $\omega, H(z)$ is all-pass. If $\phi(\omega)$ has the form $k_{0}-k_{1} \omega$, then $H(z)$ is said to have linear phase and the group delay is a constant $k_{1}$; physically, if the input to such a filter $H(z)$ has energy only in the passband of $H(z)$, then the output is a delayed version of the input, by $k_{1}$ samples. Unless mentioned otherwise, a low-pass filter has real coefficients, so that $\left|H\left(e^{j \omega}\right)\right|$ is symmetric and $\phi(\omega)$ is antisymmetric with respect to $\omega=0$. Usually $\left|H\left(\mathrm{e}^{j \omega}\right)\right|$ is plotted for $0 \leq f \leq 0.5$ (i.e., for $0 \leq \omega \leq \pi$ ). If $\omega_{p}$ and $\omega_{s}$ denote the passband and stopband edges of a low-pass filter, the quantity $\omega_{c}=\left(\omega_{p}+\omega_{s}\right) / 2$ is said to be the cutoff frequency.

Bold-faced quantities denote matrices and vectors, as in $A, H(z)$ etc. The symbol $I_{k}$ denotes the $k \times k$ identity matrix (with subscript often omitted). The quantities $\boldsymbol{A}^{\top}, \boldsymbol{A}^{\dagger}$ and $\boldsymbol{A}^{*}$ denote, respectively, the transpose, transpose conjugate, and conjugate of $\boldsymbol{A}$. For functions $\boldsymbol{H}(z)$, the notation $\boldsymbol{H}_{*}(z)$ denotes conjugation of the coefficients without conjugating $z$. For example if $H(z)=a+b z^{-1}$, then $H_{*}(z)=a^{*}+$ $b^{*} z^{-1}$. Thus, $\boldsymbol{H}^{*}(z)=\boldsymbol{H}_{*}\left(z^{*}\right)$. The notation $\tilde{\boldsymbol{H}}(\mathrm{z})$ stands for $\boldsymbol{H}_{*}^{T}\left(z^{-1}\right)$. In other words, conjugate the coefficients, take transpose (if matrix), and replace $z$ with $z^{-1}$. When $z=e^{j \omega}$ (i.e., on the unit circle), we have $\tilde{\boldsymbol{H}}(z)=\boldsymbol{H}^{\dagger}(z)$. Linear timeinvariant systems [7] are abbreviated as LTI and linear periodically time-varying systems as LPTV. A $p \times r$ matrix $\boldsymbol{A}$ is said to be unitary (orthogonal if it is real) if $\boldsymbol{A}^{\dagger} \boldsymbol{A}=\boldsymbol{c l} \boldsymbol{I}_{\text {t }}$ $c \neq 0$. Note that $\boldsymbol{A}$ is not restricted to be square. For example, $[\sin (\theta)]$ is unitary for any real $\theta$. The symbol $W_{M}$ stands for $\mathrm{e}^{-i 2 \pi / M}$. The subscript $M$ is usually deleted because its value is often clear from the context. This quantity appears in the definition of the discrete Fourier transform (DFT) [7], [8]. Thus an $M$-point sequence $\left[x_{0}, x_{1}, \cdots, x_{M-1}\right]$ has the $M$-point DFT sequence

$$
X_{k}=\sum_{n=0}^{M-1} x_{n} W^{k n}, \quad 0 \leq k \leq M-1 .
$$

The inverse DFT (IDFT) is given by

$$
x_{n}=\frac{1}{M} \sum_{k=0}^{M-1} X_{k} W^{-n k}
$$

The most crucial property of $W$ that finds repeated use in multirate DSP is the following:

$$
\sum_{n=0}^{M-1} W^{k n}= \begin{cases}M, & k=\text { integer multiple of } M \\ 0, & \text { otherwise. }\end{cases}
$$

For any pair of integers $k, n$, we have $W^{k}=W^{n}$, if and only if $k-n$ is an integer multiple of $M$. In particular, therefore, $W^{k} \neq W^{n}$ for $0 \leq k<n \leq M-1$.

State Space Descriptions: Consider a discrete time transfer matrix $\boldsymbol{H}(z)$ with input vector $u(n)$ and output vector $y(n)$. Suppose we have implemented this transfer matrix using a structure, and let $N$ denote the number of delay elements used. Label the outputs of the delay elements as the state variables $x_{k}(n), 0 \leq k \leq N-1$, and define the state vector $x(n)=\left[x_{0}(n) x_{1}(n) \cdots x_{N-1}(n)\right]^{T}$. With $u(n)$ and $y(n)$ denoting the input and output (vector) sequences to the structure, one can always find equations of the form [9]

$$
\begin{aligned}
x(n+1) & =\boldsymbol{A x}(n)+B u(n), \\
y(n) & =\mathbf{C x}(n)+\operatorname{Du}(n),
\end{aligned}
$$

to describe the structure. This is called the state-space description of the structure. The matrix $A$, called the state transition matrix, has size $N \times N$, where $N$ is the number of delays in the structure. The transfer function $H(z)$ of the structure is given by $H(z)=D+C(z I-A)^{-1} B$. The smallest number of delay elements (i.e., $z^{-1}$ elements) required to implement $\boldsymbol{H}(\mathrm{z})$ is called the McMillan degree (or simply, the degree) of $\boldsymbol{H}(z)$. If the number of delays $N$ in the structure is equal to the degree, then the structure is said to be a minimal realization of $\boldsymbol{H}(\mathrm{z})$. This is equivalent to saying that $\boldsymbol{A}$ is as small as possible.

A summary of acronyms and common notations used in this paper is found in the Nomenclature, which follows section XI.

\section{Basic Building Blocks and ToOls}

In this section we introduce the basic multirate building blocks, along with their frequency-domain characterizations, and interconnection behaviors.

\section{A. Decimators and Interpolators}

Fig. 1 shows block diagrams of these building blocks. The decimator is characterized by the input-output relation

$$
\begin{aligned}
& y_{D}(n)=x(M n) \\
& x(n) \rightarrow y_{D}(n) \\
& \text { (a) }
\end{aligned}
$$

Fig. 1. Building blocks. (a) $M$-fold decimator. (b) $L$-fold interpolator.

which says that the output at time $n$ is equal to the input at time $M n$. As a consequence, only the input samples with sample numbers equal to a multiple of $M$ are retained. This sampling-rate reduction by a factor of $M$ is demonstrated in Fig. 2 for the case of $M=2$. The $L$-fold interpolator is char- 


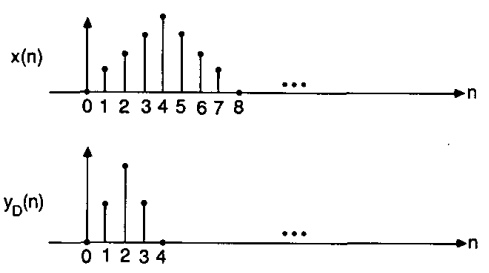

Fig. 2. Demonstration of decimation for $M=2$.

acterized by the input-output relation

$$
y_{N}(n)= \begin{cases}x\left(\frac{n}{L}\right) & \text { if } n \text { is a multiple of } L \\ 0 & \text { otherwise. }\end{cases}
$$

That is, the output $y_{l}(n)$ is obtained by inserting $L-1$ zerovalued samples between adjacent samples of $x(n)$, as demonstrated in Fig. 3 for $L=2$. The decimator and interpolator

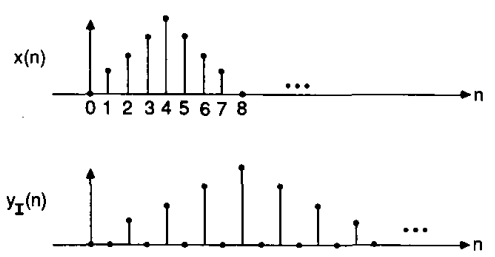

Fig. 3. Demonstration of interpolation for $L=2$.

are linear systems even though they are time-varying [4], [5], [10].

The $z$ transform of the interpolator output $y_{l}(n)$ is given by [4]:

$$
Y_{l}(z)=X\left(z^{l}\right)
$$

This means $Y_{l}\left(\mathrm{e}^{j \omega}\right)=X\left(\mathrm{e}^{j \omega L}\right)$ i.e., $Y_{l}\left(e^{j \omega}\right)$ is an $L$-fold compressed version of $X\left(\mathrm{e}^{j \omega}\right)$, as demonstrated in Fig. $4(\mathrm{~b})$. The appearance of multiple copies of the basic spectrum in Fig. 4 is called the imaging effect and the extra copies are the images created by the interpolator.

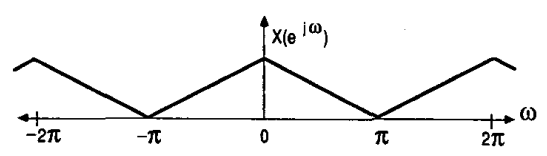

(a)

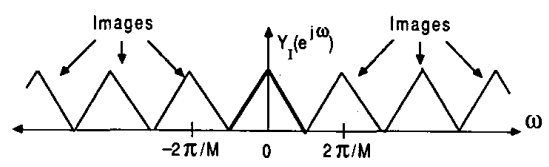

(b)

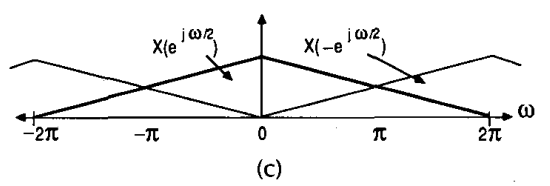

Fig. 4. Transform-domain effects of decimation and interpolation. (a) The $z$ transform. (b) L-fold compressed version. (c) Demonstration of effect when $M=2$.
Since decimation corresponds to compression in the time domain, one might expect a stretching effect in the frequency domain. To be more precise, the $z$ transform of $y_{D}(n)$ is given by

$$
Y_{D}(z)=\frac{1}{M} \sum_{k=0}^{M-1} X\left(z^{1 / M} W^{k}\right)
$$

which means $M Y_{D}\left(\mathrm{e}^{j \omega}\right)=\sum_{k=0}^{M-1} X\left(\mathrm{e}^{j(\omega-2 \pi k) / M}\right)$. The term with $k=0$ is indeed the $M$-fold stretched version of $X\left(\mathrm{e}^{j \omega}\right)$. The $M-1$ terms with $k>0$ are uniformly shifted versions of this stretched version. These $M$ terms together make up a function with period $2 \pi$ in $\omega$, which is the basic property of the Fourier transform of any sequence [8]. Fig. 4(c) demonstrates this effect for $M=2$. The terms with $k>0$ are called the aliasing terms. As long as $x(n)$ is bandlimited to $|\omega|<\pi / M$, there is no overlap of these terms with the $k=$ 0 term.

The fundamental difference between aliasing and imaging is important to notice. Aliasing can cause loss of information because of the possible overlap of the shifted versions of the stretched version of $X\left(e^{j \omega}\right)$. Imaging, on the other hand, does not lead to any loss of information (which is consistent with the fact that no time-domain samples are lost).

\section{B. Interconnections}

Fig. 5 shows a cascade connection which is often encountered in filter-bank systems. The signal $v(n)$ here is equal to

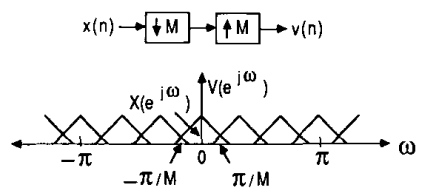

Fig. 5. Effect of decimation followed by interpolation.

$x(n)$ whenever $n$ is a multiple of $M$, and zero otherwise. The transform-domain relation is

$$
V(z)=\frac{1}{M} \sum_{k=0}^{M-1} X\left(z W^{k}\right)
$$

which means that $M V\left(e^{j \omega}\right)$ is a sum of $X\left(e^{j \omega}\right)$ with the $M$ 1 uniformly shifted versions $X\left(e^{j(\omega-2 \pi k / M)}\right)$. From the figure we see that $x(n)$ can be recovered from $v(n)$ by eliminating the images by filtering, provided none of the images has an overlap with $X\left(e^{j \omega}\right)$. If such an overlap occurs, it implies aliasing and $x(n)$ cannot be recovered. Notice that in order for $x(n)$ to be recoverable it is not necessary for $X\left(\mathrm{e}^{j \omega}\right)$ to be restricted to $|\omega|<\pi / M$. It is sufficient for the total bandwid th of $X\left(e^{j \omega}\right)$ to be less than $2 \pi / M$. Thus a general bandpass signal with energy in the region $a \leq \omega \leq a+2 \pi / M$ can be decimated by $M$ without creating overlap of the alias components, and the decimated signal in general is a full-band signal.

A different type of cascade is shown in Fig. 6(a). We shall have occasion to use this in section IV-B, which concentrates on multileve!-polyphase decompositions. It should be cautioned that the two building blocks in Fig. 6(a) are not, in general, interchangeable, i.e., the systems in Fig. 6(a) and $6(\mathrm{~b})$ are not equivalent. For example, with $M=L$, the system of Fig. 6(a) is an identity system, whereas the system of Fig. $6(b)$ causes a loss of $M-1$ out of $M$ samples. It can be shown 


$$
x(n) \longrightarrow \varphi L^{x_{1}(n)} \cdot \downarrow M \rightarrow y_{1}(n)
$$

(a)

$$
x(n) \rightarrow \operatorname{IM}^{x_{2}(n)}, \uparrow L, y_{2}(n)
$$

(b)

Fig. 6. Two ways to cascade decimator and interpolator. These are equivalent if and only if $M$ and $L$ are relatively prime. (a) Example of identity system. (b) Example of loss of $M-1$ out of $M$ samples.

(Appendix A) that the systems of Fig. 6(a) and 6(b) are identical if and only if $L$ and $M$ are relatively prime.

Decimation Filters and Interpolation Filters: In most applications a decimator is preceded by a bandlimiting filter $H(z)$ whose purpose is to avoid aliasing. For example, a low-pass filter with stopband edge $\omega_{s}=\pi / M$ can serve as such a filter. The cascade shown in Fig. 7 (a) is commonly called a decimation filter. An interpolation filter, on the other hand, is a device which follows an interpolator (Fig. 7(b)), the purpose being to eliminate the images. The lowpass filter of Fig. 7 (c) again serves as an example (with $L=$ $M)$.

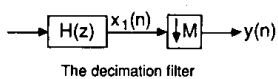

(a)

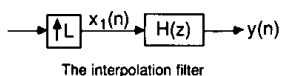

(b)

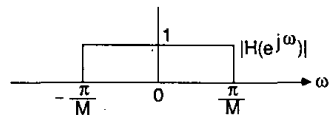

(c)

Fig. 7. (a) Decimation filter. (b) Interpolation filter. (c) Lowpass filter.

Fractional Sampling Rate Alterations: Fig. 8(a) shows a scheme for reducing the sampling rate by a nonintegral (rational) number M/L. Fractional reduction of sampling rate often results in data compression without loss of information. As an example, if $X\left(e^{j \omega}\right)$ is as in Fig. $8(\mathrm{~b})$, then a fractional reduction by $3 / 2$ is possible. This can be accom-

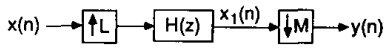

(a)

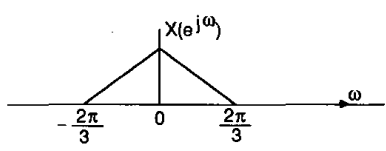

(b)

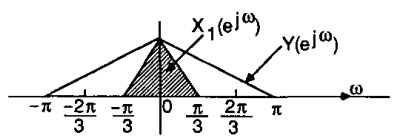

(c)

Fig. 8. Decimation by rational fraction of $M / L$. (a) General structure. (b) Example of bandlimited signal. (c) Effect of fractional decimation of this signal $(L=2, M=3)$. plished by setting $M=3, L=2$ in Fig. $8(a)$. The filter $H(z)$ is then taken to be low pass, with passband edge at $\pi / 3$ and stopband edge at $2 \pi / 3$. Notice that in this application, the transition bandwidth of $H(z)$ need not be unduly narrow. The various signals in Fig. 8(a) have transforms as in Fig. 8(c), so that $Y\left(e^{j \omega}\right)$ is a fractionally stretched version of $X\left(e^{j \omega}\right)$.

Two Noble Identities: In Fig. 9(a) we have a decimator followed by a transfer function $G(z)$. It can be proved, based

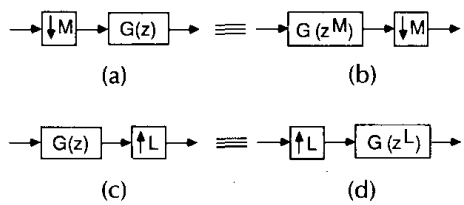

Fig 9. Noble identities for multirate systems. (a) Decimator followed by transfer function $G(z)$. (b) Equivalent cascade. (c) Example of transfer function preceding. (d) Equivalent cascade.

on (3b), that this cascade is equivalent to the one in Fig. $9(\mathrm{~b})$ provided $G(z)$ is a rational transfer function (i.e., a ratio of polynomials in $\left.z^{-1}\right)$. In a similar manner, the two cascades in Figs. 9(c) and 9(d) are equivalent (provided $G(z)$ is rational), as can be proved from (3a). These identities are very valuable in practically all applications for efficient implementation of filters and filter banks. We shall call these the "noble identities."

\section{Analysis and Synthesis Banks}

These are the two basic types of filter banks. An analysis bank is a set of analysis filters $H_{k}(z)$ which splits a signal into $M$ subband signals $x_{k}(n)$ as shown in Fig. 10(a). What we do

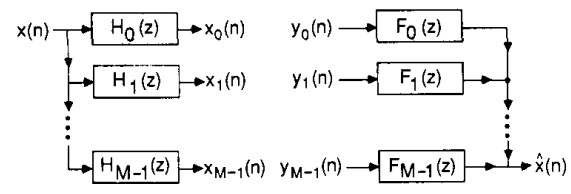

(a)

(b)

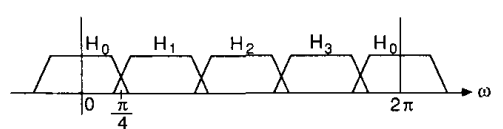

(c)

Fig. 10. Analysis and synthesis filter banks. (a) Analysis bank. (b) Synthesis bank. (c) Typical response of uniform DFT filter bank; here $M=4$.

with the subband signals depends on the application, as we shall see in sections III, VI, and IX. Next, a synthesis bank (Fig. 10(b)) consists of $M$ synthesis filters $F_{k}(z)$, which combine $M$ signals $y_{k}(n)$ (possibly from an analysis bank) into a reconstructed signal $\hat{x}(n)$. There are several types of filter banks, i.e., the complementary type, the Nyquist type, etc., to be described in Section $V$ along with applications.

Uniform DFT Filter Banks: An analysis bank with $M$ filters $(M>1)$ is said to be a uniform DFT filter bank if all the filters are derived from $H_{0}(z)$ according to $H_{k}(z)=H_{0}\left(z W^{k}\right), 0 \leq$ $k \leq M-1$. Here $H_{0}(z)$ is called the prototype filter. Note 
that $H_{k}\left(\mathrm{e}^{j \omega}\right)=H_{0}\left(\mathrm{e}^{j(\omega-2 \pi k / M)}\right)$, which means that the frequency responses of $H_{k}(z)$ are uniformly shifted versions of $H_{0}\left(\mathrm{e}^{j \omega}\right)$. Fig. 10(c) shows a typical set of responses, where $H_{0}(z)$ is taken to be low pass. More details can be found in section IV-C and in [4] and [11].

\section{Some Applications of Multirate Systems}

We shall now review a number of important applications of multirate filters and filter banks, with pointers to the literature for details, examples, and demonstrations. In section IX, several unconventional applications are also outlined.

Applications in the design of transmultiplexers (which are devices for conversion between frequency division multiplexing (FDM) and time-division multiplexing (TDM)) are not discussed here in detail, primarily because of the excellent treatment already available in [13]. Also see [14] for the correspondence between transmultiplexers and analysis/synthesis filter banks. The input to a TDM-to-FDM converter is a signal $y(n)$, which is the time-multiplexed version of $M$ signals $y_{k}(n), 0 \leq k \leq M-1$. Given $y(n)$, the components $y_{k}(n)$ can easily be separated out by use of a commutator switch [4], [13]. These $M$ signals are then modulated using distinct carrier frequencies. The carrier frequencies $\omega_{k}, 0 \leq k \leq M-1$ are chosen so that there is sufficient spectral gap between the messages. A sum of these $M$ signals (which is the FDM signal) is then transmitted through the channel. The total channel bandwidth is therefore required to exceed the sum of signal bandwidths because of the safeguard gap between adjacent spectra. The gap enables one to obtain perfect recovery of the multiplexed signals $y_{k}(n)$ at a future point.

A novel approach to transmultiplexing was suggested in [36] and cited in [14], based on synthesis and analysis filter banks. This approach permits overlap between the spectra of successive messages in the frequency domain. The total required channel bandwidth is therefore less than that in conventional FDM channels. Conditions are derived under which cross-talk can be avoided and the set of $M$ original signals can still be recovered from this version. Details can be found in reference [36] cited in [14].

\section{A. Digital Audio Systems}

In the digital audio industry, it is a common requirement to change the sampling rates of band-limited sequences. This arises for example when an analog music waveform $x_{a}(t)$ is to be digitized. Assuming that the significant information is in the band $0 \leq|\Omega| / 2 \pi \leq 22 \mathrm{kHz}$ [15], a minimum sampling rate of $44 \mathrm{kHz}$ is suggested (Fig. 11(a)). It is, however, necessary to perform analog filtering before sampling to eliminate aliasing of out-of-band noise. Now the requirements on the analog filter $H_{a}(j \Omega)$ (Fig. 11(b)) are strigent: it should have a fairly flat passband (so that $X_{a}(j \Omega)$ is not distorted) and a narrow transition band (so that only a small amount of unwanted energy is let in). Optimal filters for this purpose (such as elliptic filters [9], which are optimal in the minimax sense) have a very nonlinear phase response [16, page 82] around the bandedge (i.e., around $22 \mathrm{kHz}$ ). In highquality music this is considered to be objectionable [15]. A common strategy to solve this problem is to oversample $x_{a}(t)$ by a factor of two (and often four). The filter $H_{a}(j \Omega)$ now has a much wider transition band, as in Fig. 11(c), so that

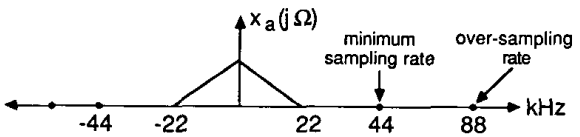

(a)

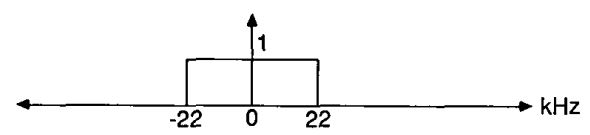

(b)

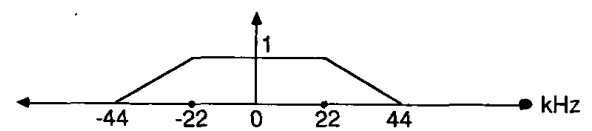

(c)

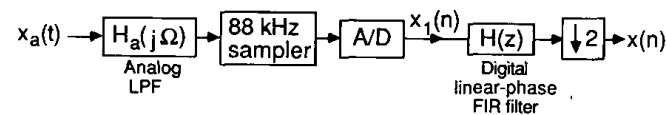

(d)

Fig. 11. (a) Spectrum of $x_{a}(t)$. (b) Antialiasing filter response for sampling at $44 \mathrm{kHz}$. (c) Antialiasing filter response for sampling at $88 \mathrm{kHz}$. (d) Improved scheme for A/D stage of digital audio system.

the phase-response nonlinearity is acceptably low. A simple analog Bessel filter (which has linear phase in the passband [9]) can be used in practice. The sequence $x_{1}(n)$ so generated is then lowpass filtered (Fig. 11(d)) by a digital filter $H(z)$ and then decimated by the same factor of two to obtain the final digital signal $x(n)$. The crucial point is that since $H(z)$ is digital, it can be designed to have linear phase [7], [16], [17], while at the same time providing the desired degree of sharpness.

A similar problem arises after the D/A conversion stage, where the digital music signal $y(n)$ should be converted to an analog signal by lowpass filtering. To eliminate the images of $Y\left(e^{i \omega}\right)$ in the region outside $22 \mathrm{kHz}$, a sharp cutoff (hence nonlinear phase) analog low-pass filter is required. This problem is avoided by using an interpolation filter, as in Fig. 7(b), which increases the sampling rate digitally. After this, D/A conversion is performed followed by analog filtering. The interpolation filter $H(z)$ is once again a linearphase FIR low-pass filter and introduces no phase distortion.

The obvious price paid in these systems is the increased internal rate of computation. However, by using the polyphase framework (section IV) the efficiency of these multirate systems can be dramatically improved.

In digital audio, it is relatively economic (compared to the analog case) to produce high-quality copies of material from one medium to another [15]. Perhaps to discourage such practice, the sampling rates used for various media are often made different from each other. It is therefore necessary in studios to design efficient nonintegral sampling rate converters (such as the one in Fig. 8(a)). See section IV-B for further details. Further applications of multirate filter banks in digital audio can be found in section V-E.

\section{B. Subband Coding of Speech and Image Signals}

In practice, one often encounters signals with energy dominantly concentrated in a particular region of fre- 
quency. An extreme example was shown in Fig. 8(b), where all the energy is in $0 \leq|\omega|<2 \pi / 3$. In this case it is possible to compress the signal simply by decimating it by a factor of $3 / 2$ (using Fig. 8(a)) or less.

It is more common, however, to encounter signals that are not bandlimited but still have dominant frequency bands. An example is shown in Fig. 12(a). The information

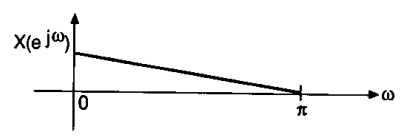

(a)

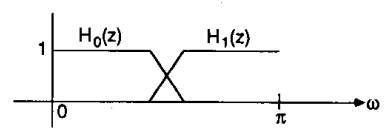

(b)

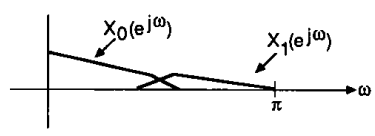

(c)

Fig. 12. Splitting a signal into subband signals $x_{0}(n)$ and $x_{1}(n)$. (a) Case of dominant frequency bands. (b) Splitting the signal by using an analysis bank with $M=2$ (Fig. 10(a)). (c) Encoding the subband signal.

in $|\omega|>\pi / 2$ is not small enough to be discarded. And we cannot decimate $x(n)$ without causing aliasing either. It does seem unfortunate that a small (but not negligible) fraction. of energy in the high-frequency region should prevent us from obtaining any kind of signal compression at all.

But there is a way to get around this difficulty: we can split the signal into two frequency bands by using an analysis bank (Fig. 10(a) with $M=2$ ), with responses as in Fig. 12(b). The subband signal $x_{1}(n)$ has less energy than $x_{0}(n)$, and so can be encoded with fewer bits than $x_{0}(n)$. As an example, let $x(n)$ be a $10-\mathrm{kHz}$ signal (10 000 samples/s) normally requiring 8 bits per sample so that the data rate is $80 \mathrm{kbits} /$ s. Let us assume that the subband signals $x_{0}(n)$ and $x_{1}(n)$ can be represented with 8 bits and 4 bits per sample, respectively. Because these signals are also decimated by two, the data rate now works out to be $40+20=60 \mathrm{kbits} / \mathrm{s}$, which is a compression by $4 / 3$. This is the basic principle of subband coding: split the signal into two or more subbands, decimate each subband signal, and allocate bits for samples in each subband depending on the energy content. This strategy is clearly a generalization of the simple decimation process (which works only for strictly bandlimited signals). In speech coding practice, further use of the perceptive properties in each subband is exploited before quantization [18]-[21].

The reconstruction of the full band signal is done using the interpolators and synthesis bank filters as in Fig. 13. The

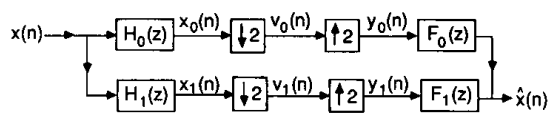

Fig. 13. Analysis/synthesis system for subband coding. (Also called two-band QMF bank; see text.) interpolators restore the original sampling rate, and the filters $F_{k}(z)$ eliminate the images. Further generalizations follow immediately: the signal can be split into $M$ subbands with each subband signal decimated by $M$ and independently quantized.

Pioneering work on the application of this technique in speech coding has been done by Crochiere [18], [19] and by Galand and Estaban [21]. The coding in each subband is typically more sophisticated than just quantization. For example, techniques such as adaptive pulse code modulation (APCM) and adaptive delta pulse code modulation (ADPCM) are commonly used [20]. The specific properties of speech signals and their relation to human perception are carefully exploited in the coding process; the appropriate number of subbands and the coding accuracy in each subband are judged based on the articulation index. See [18]-[20] for complete examples of speech coding, using this idea. The quality of subband coders is usually judged by what is called the mean opinion score (MOS). This score is obtained by performing listening tests with the help of a wide variety of unbiased listeners, and asking them to assign a score for the quality of the reproduced signal $\hat{x}(n)$ (in comparison to $x(n))$. The maximum score is normalized to 5 by convention. Subband-coded speech with an average bit rate of $16 \mathrm{kbits} / \mathrm{s}$ can typically achieve a MOS of 3.1, whereas at $32 \mathrm{kbits} / \mathrm{s}$ a score of 4.3 has been achieved in the past [ 20 , chapter 11].

In video signal processing, subband coding has been applied for image compression, and success has been reported by several authors [22]-[27]. The results in [22], in particular, use vector quantization [28] in each subband to obtain a coded image with only 0.48 bits per pixel. (The original uncoded picture being an 8 bits per pixel image.)

Several comments are now in order: first, in order for subband coding to work, it is necessary to have some a priori knowledge about the energy distribution of $X\left(e^{j \omega}\right)$. In speech and image processing, for example, such knowledge is usually available because of the long history of experience with the coding of such signals. Second, the bandsplitting and decimation operation inevitably results in aliasing because the filters $H_{k}(z)$ are not ideal. The filters $F_{k}(z)$ should be chosen carefully in such a way that aliasing is actually canceled (see section VI, where we include a detailed review of al! the distortions which arise in the analysis/synthesis system).

\section{Analog Voice Privacy Systems}

These systems [29] are intended to communicate speech over standard analog telephone links, while at the same time ensuring voice privacy. The main idea is to split a signal $x(n)$ into $M$ subband signals $x_{k}(n)$ and then divide each subband signal into segments in the time domain. These segments of subband signals are then permuted and recombined into a single encrypted signal $y(n)$, which can then be transmitted (after D/A conversion). For example, if there are five subbands and twenty-five time segments in each subband, then there are 125 ! possible permutations, and unless an eavesdropper has the key for decryption, he will be unable to perform a pleasant job of eavesdropping. The aims of the designer of such a privacy system are: the encrypted message should be unintelligible, decryption without a key should be very difficult, and the decrypted signal should 
be of good quality retaining naturalness and voice characteristics. These features have indeed been achieved by Cox et al. [29].

At the receiver end, $y(n)$ is again split into subbands, and the time-segments of the subbands unshuffled to get $x_{k}(n)$, which can then be interpolated and recombined through the synthesis filters.

\section{The Polyphase Decomposition and Its Applications}

The polyphase decomposition, which originated from the work by Bellanger et al. [11], is very fundamental to many applications in multirate DSP. These include efficient realtime implementation of decimation and interpolation filters, fractional sampling-rate changing devices, uniform DFT filter banks, and perfect-reconstruction analysis/synthesis systems. More recently, the polyphase idea has been applied for the derivation of new sampling theorems [30], and for recovering bandlimited signals from non-uniformly sampled versions. In this section the basic concept is introduced and some of the aforementioned applications are elaborated. Section IX deals with the remaining applications. To avoid repetitions, results presented in [3], [4] are reviewed only briefly here.

\section{A. The Polyphase Decomposition}

Let $H(z)=\Sigma_{n=-\infty}^{\infty} h(n) z^{-n}$ be a transfer function representing a digital filter. We can rewrite $H(z)$ in the form

$$
\begin{aligned}
H(z)= & {\left[\cdots+h(-4) z^{4}+h(-2) z^{2}+h(0)\right.} \\
& \left.+h(2) z^{-2}+h(4) z^{-4}+\cdots\right] \\
& +z^{-1}\left[\cdots+h(-3) z^{4}+h(-1) z^{2}\right. \\
& \left.+h(1)+h(3) z^{-2}+\cdots\right] .
\end{aligned}
$$

If we use the abbreviations

$$
E_{0}(z)=\sum_{n=-\infty}^{\infty} h(2 n) z^{-n}, \quad E_{1}(z)=\sum_{n=-\infty}^{\infty} h(2 n+1) z^{-n}
$$

we can re-express (5) in the form

$$
H(z)=E_{0}\left(z^{2}\right)+z^{-1} E_{1}\left(z^{2}\right) .
$$

Basically, we have merely grouped the impulse-response coefficients $h(n)$ into even numbered samples $e_{0}(n)=h(2 n)$ and odd-numbered samples $e_{1}(n)=h(2 n+1)$. Denoting the $z$-transforms of $e_{0}(n)$ and $e_{1}(n)$ by $E_{0}(z)$ and $E_{1}(z)$, respectively, we obtain the relation (7). This representation will be called the two-component polyphase decomposition of $H(z)$. The quantities $E_{0}(z)$ and $E_{1}(z)$ are called the polyphase components of $H(z)$. As an example, let $H(z)=1+2 z^{-1}+5 z^{-2}+$ $4 z^{-3}$. Then $H(z)=1+5 z^{-2}+z^{-1}\left[2+4 z^{-2}\right]$, so that $E_{0}(z)=$ $1+5 z^{-1}$ and $E_{1}(z)=2+4 z^{-1}$.

Note that the representation of (7) holds whether $H(z)$ is FIR or IIR. For example, let

$$
H(z)=\frac{1}{1-a z^{-1}} .
$$

By using the identity $1-x=\left(1-x^{2}\right) /(1+x)$, we can rewrite (8) as

$$
H(z)=\frac{1}{1-a^{2} z^{-2}}+\frac{a z^{-1}}{1-a^{2} z^{-2}}
$$

so that $E_{0}(z)=1 /\left(1-a^{2} z^{-1}\right)$ and $E_{1}(z)=a /\left(1-a^{2} z^{-1}\right)$.
In an exactly analogous manner, it is possible to represent $H(z)$ in the $M$-component polyphase form

$$
H(z)=\sum_{k=0}^{M-1} z^{-k} E_{k}\left(z^{M}\right) .
$$

Once again $E_{k}(z)$ are called polyphase components and the coefficients $e_{k}(n)$ and $E_{k}(z)$ are given by

$$
\mathrm{e}_{k}(n)=h(n M+k), \quad 0 \leq k \leq M-1 .
$$

In other words, the impulse response sequence $\{h(n)\}$ has now been grouped into $M$ subsequences $e_{k}(n)$. The $k$ th subsequence $e_{k}(n)$ is mererly the $M$-fold decimated version of $h(n+k)$. Note that for a given $H(z)$, the quantity $E_{k}(z)$ depends on $M$. For example $E_{0}(z)$ in $(7)$ is totally different from that in (10). A second subscript (such as $E_{k, M}(\mathrm{z})$ ), which would make this distinction clear, is avoided in this paper in the interest of simplicity. In most applications $M$ is fixed, and there is no room for confusion.

An important property of the representation (10) is given by the following relation:

$$
\sum_{k=0}^{M-1} H\left(z W^{k}\right)=M E_{0}\left(z^{M}\right)
$$

which follows from the property (1c) of the quantity $W$. The $z$ transform $E_{0}(z)$ of $e_{0}(n)$ is thus seen to be equal to $\Sigma_{k=0}^{M-1}$ $H\left(z^{1 / M} W^{k}\right) / M$, and since $e_{0}(n)$ is nothing but the $M$-fold decimated version of $h(n)$, this gives a proof of the formula (3b)!

Before getting into applications, we shall mention a second type of polyphase decomposition which is notationally more convenient in some situations. This is given by

$$
H(z)=\sum_{k=0}^{M-1} z^{-(M-1-k)} R_{k}\left(z^{M}\right) .
$$

It is evident that $R_{k}(z)$ is related to $E_{k}(z)$ by $R_{k}(z)=E_{M-1-k}(z)$, which is just a renumbering of the components. The representations (10) and (13) will be, respectively, called Type 1 and Type 2 polyphase decompositions. Correspondingly, $E_{k}(z)$ and $R_{k}(z), 0 \leq k \leq M-1$ are called Type 1 and Type 2 polyphase components. It is important to notice that the polyphase decomposition can be applied for any sequence $x(n)$ and is not restricted to impulse response coefficients.

\section{B. Application in Sampling-Rate Alteration}

In Section II-B we mentioned three standard kinds of sampling-rate alterations, namely decimation, interpolation, and fractional decimation. Each of these involves a digital filter $H(z)$, which unfortunately operates at a point where the sampling rate is highest (see Figs. 7(a), 7(b) and 8(a)). These filtering operations can be made more efficient as described next.

Decimation Filters: Consider again the decimation filter of Fig. 7(a) with $M=2$. Suppose we implement $H(z)$ (assumed FIR of length $N$ ) directly as in Fig. 14. The system has to compute only the even numbered output samples $\gamma(2 n)$. For each computed sample we require $N$ multiplications and $N-1$ additions. Assume that $x(n)$ is arriving in real-time at the rate

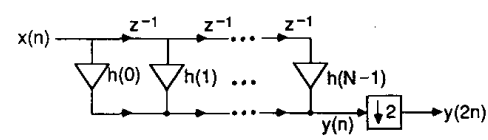

Fig. 14. Direct implementation of FIR decimation filter. 
of one sample per unit time. The scheme of Fig. 14 would then require that the $N$ multiplications and $N-1$ additions be performed in one unit of time (because, as the next input sample arrives, the internal delay elements $z^{-1}$ are updated to the new set of values). So, whenever $y(2 n)$ is being computed, the system is working at the rate of $N \mathrm{MPU}$ and $N$ - 1 APU. ${ }^{1}$ And at odd intervals, the system is sitting idle (because $y(2 n+1)$ need not be computed). This is clearly an inefficient use of computing resources.

On the other hand, if we implement the system in polyphase form, we can redraw Fig. 7(a) as in Fig. 15(a). Next,

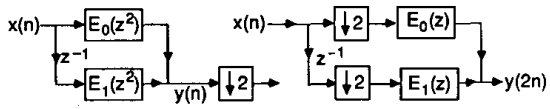

(a)

(b)

Fig. 15. Polyphase implementation of decimation filter. (a) Redrawing based on Fig. 7(a). (b) Redrawing by use of first noble identity (Fig. 9).

with the help of the first noble identity (Fig. 9) we can redraw Fig. 15(a) as in Fig. 15(b). Now the building blocks $E_{0}(z)$ and $E_{1}(z)$ are receiving the samples at half the rate (i.e., one sample in two units of time). We therefore need to perform only $\mathrm{N} / 2 \mathrm{MPUs}$ and $(\mathrm{N}-1) / 2 \mathrm{APUs}$ on the average. The polyphase representation is thus a neat tool which enables us to rearrange the computations of the filtering operation, so as to minimize the computational load per unit time. Looking at it in another way, each multiplier in Fig. 14 is required to complete its operation in one unit of time, whereas in Fig. 15(b), two units of time are available for the same job.

For general $M$, the decimation filter can be implemented using the $M$-component decomposition of (10). The computational saving is $M$-fold.

Interpolation Filters: Recall the interpolation filter of Fig. $7(b)$. Assume first that $H(z)$ is directly implemented as in Fig. 14. The input $x_{1}(n)$ to the filter has all the odd numbered samples equal to zero. As a result, only about 50 percent of the multipliers in Fig. 14 have nonzero inputs. As such, the implementation does not exploit this fact to minimize the required computation per unit time. For example, when $n$ is even, $h(0)$ has to work at two times the input rate (because its input will change after this duration), while at the same time $h(1)$ is resting. Once again a polyphase approach will give a more efficient design. For this we use (13) with $M=2$, and invoke the second noble identity (Fig. 9) to obtain the implementation of Fig. 16. Here each mul-

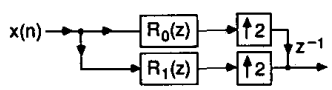

Fig. 16. Polyphase implementation of interpolation filter.

tiplier in each building block is constantly occupied, and gets two units of time to finish its job.

Commutator Models: A very convenient way to represent the polyphase decomposition is offered by the commutator

\footnotetext{
${ }^{1}$ The abbreviations MPU and APU represent multiplications per unit time and additions per unit time, respectively, where a unit of time is the separation between the samples of the input signal $x(n)$
}

switch model. The basic principles of this are discussed clearly in [3] and [4]. The applications of the commutator model in multirate analysis/synthesis systems is also indicated in [31]. It can be verified that the Type-1 polyphase decomposition can be represented using the counterclockwise commutator model [4] and the Type-2 polyphase using the clockwise model. In this paper we shall not make explicit reference to this type of a model, and to minimize duplication of the results described in [3] and [4], we refrain from further elaboration.

Fractional Decimators: Consider Fig. 8(a), which is a scheme to obtain a sampling rate reduction by the factor $M / L$. If we implement $H(z)$ directly, as in Fig. 14, then it is inefficient due to two reasons: first the input to $H(z)$ contains $L-1$ zero-valued samples out of every $L$ samples so that only one out of $L$ multipliers is working at a time. Second, only one out of $M$ computed samples in $x_{1}(n)$ is going to be used. These two factors are related to the fact that the computations in Fig. 8(a) are done at the point where the sampling rate is the highest possible.

To obtain a more efficient implementation, we begin by considering the $M=3, L=2$ example again. By using the Type-1 polyphase representation and rearranging as we did in Fig. 15, we obtain the implementation of Fig. 17(a). On

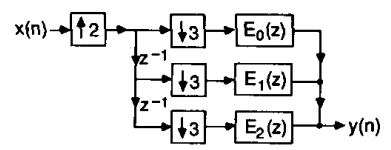

(a)

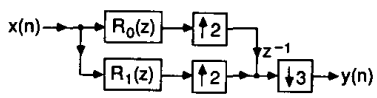

(b)

Fig. 17. Two ways to improve the efficiency of fractionaldecimation filter. (a) Using Type-1 decomposition, based on Fig. 15. (b) Using Type-2 decomposition, based on Fig. 16.

the other hand, if we use the Type- 2 decomposition and rearrange as in Fig. 16, we get Fig. 17(b). Clearly Fig. 17(a) and Fig. 17(b) are more efficient than Fig. 8(a), by factors of $M$ and $L$, respectively. Can we do better than these? Notice that Fig. 17(a) exploits the presence of the decimator whereas Fig. 17(b) exploits the presence of the interpolator. Is it possible to exploit both?

The answer, indeed, is in the affirmative. Such an efficient scheme is obtained in [3, page 311] by the use of filter structures with time-varying coefficients. The derivation in [3] is based on time-domain reasonings.

We shall now describe an implementation with the highest possible efficiency, based on z-domain reasonings [32] using the polyphase decomposition. First notice that we are stuck in Fig. 17 mainly because we cannot move the interpolator any more to the right (and decimator any more to the left). This is because the noble identities simply cannot be applied anymore! However, here is the nice trick which comes to our rescue: we can write $z^{-1}=z^{-3} z^{2}$ so that Fig. 17 (b) can be redrawn as in Fig. 18(a). With the help of the noble identities, this becomes Fig. 18(b). Next we can interchange the decimator with the interpolator (which is valid because 2 and 3 are relatively prime; see section II-B) to 


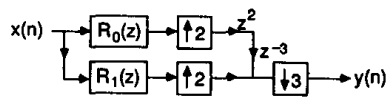

(a)

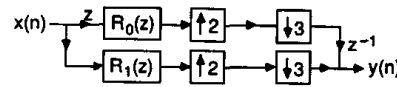

(b)

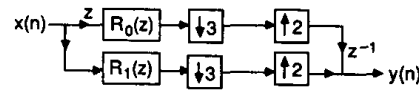

(c)

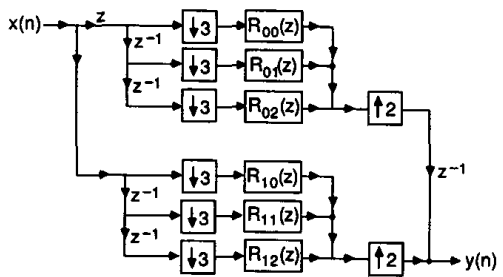

(d)

Fig. 18. Successive redrawings of fractional decimation circuit, to maximize computational efficiency. (a) First redrawing, when $z^{-1}=z^{-3} z^{2}$. (b) Second redrawing, using noble identities. (c) Third redrawing, interchanging decimator with interpolator. (d) Fourth redrawing, using Type-1 polyphase decomposition (equation (14)).

obtain Fig. 18(c). Finally, we can perform a Type-1 polyphase decomposition of the polyphase components $R_{0}(z)$ and $R_{1}(z)$ as follows:

$$
\begin{aligned}
& R_{0}(z)=R_{00}\left(z^{3}\right)+z^{-1} R_{01}\left(z^{3}\right)+z^{-2} R_{02}\left(z^{3}\right), \\
& R_{1}(z)=R_{10}\left(z^{3}\right)+z^{-1} R_{11}\left(z^{3}\right)+z^{-2} R_{12}\left(z^{3}\right)
\end{aligned}
$$

so that Fig. 18(c) can be redrawn as in Fig. 18(d). In summary, Fig. $18(d)$ is equivalent to Fig. $8(\mathrm{a})$ ! If $H(z)=\Sigma_{n=0}^{N-1} h(n) z^{-n}$, then we still have only $N$ multipliers in Fig. 18(d). However, each multiplier operates at the lowest possible rate, which is one-third of the input rate. This trick works for arbitrary $M, L$ as long as they are relatively prime, because in that case, two things are true: a) there exist integers $n_{0}$ and $n_{1}$ satisfying $-n_{0} L+n_{1} M=1$ (Euclid's theorem, [33]) so that we can replace each $z^{-1}$ with $z^{n_{0} L} z^{-n_{1} M}$, and b) the decimator and interpolator can be interchanged.

\section{Application in Filter-Bank Implementations}

Consider an analysis filter bank with two filters (i.e., $M$ $=2$ in Fig. 10(a)) and let the filters be related as $H_{1}(z)=H_{0}(-z)$ (i.e., a uniform DFT bank with $M=2$ ). If we express $H_{0}(z)$ as in (7), then we have $H_{1}(z)=E_{0}\left(z^{2}\right)-z^{-1} E_{1}\left(z^{2}\right)$, so that we can implement the system as in Fig. 19. If the implementation of $H_{0}(z)$ requires $N_{m}$ multiplications and $N_{a}$ additions, then the pair $\left[H_{0}(z), H_{1}(z)\right]$, implemented as in the preceding, requires only $N_{m}$ multiplications and $N_{a}+1$ additions. We therefore obtain two filters at nearly the cost of one.

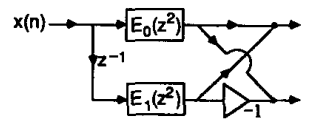

Fig. 19. Implementing pair $\left[H_{0}(z), H_{0}(-z)\right]$
More generally, consider the uniform DFT filter bank described in setion II-C with $H_{k}(z)=H_{0}\left(z W^{k}\right)$. Expressing the prototype $H_{0}(z)$ as in $(10)$ we see that

$$
H_{k}(z)=\sum_{n=0}^{M-1} W^{-k n} z^{-n} E_{n}\left(z^{M}\right)
$$

which follows because $W^{M}=1$. Notice the similarity of the summation (15) to the IDFT relation in (1b). This enables us to draw the complete analysis bank as in Fig. 20. The total

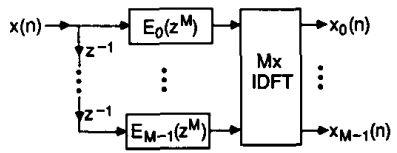

Fig. 20. Implementing uniform DFT bank using polyphase decomposition.

computational complexity of this structure, proposed by Bellanger et al., is equal to that of the prototype plus the cost of the IDFT operation. For large $M$, a standard FFT can be used to minimize the cost of the IDFT. For special values of $M$, such as $M=2,4$, etc., the IDFT operation is entirely multiplierless. In any case, it is clear that the structure of Fig. 20 is much more efficient than a direct implementation of $M$ filters, because we obtain $M$ filters at the cost of one filter (plus the IDFT overhead). In fact, in most applications, the outputs $x_{k}(n)$ of the filters $H_{k}(z)$ are decimated by $M$. By using the appropriate noble identity we can move these decimators to the left as shown in Fig. 21. We now see that,

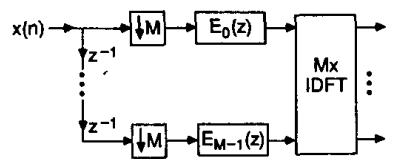

Fig. 21. Redrawing Fig. 20 when $x_{k}(n)$ must be decimated.

apart from the IDFT overhead, the number of MPUs and APUs of the entire system of $M$ filters with decimation is $1 / M$ th that of a single filter $H_{0}(z)$ without decimation! In other words, the complexity tends to decrease as $M$ increases. This dramatic result is a consequence of the polyphase representation, combined with decimation. The uniform DFT analysis bank is therefore an efficient tool for spectrum analysis, and has actually been used in commercial spectrum analyzers. See, for example, the recent Tektronix product described in [34].

There are, however, some applications for which the uniform DFT filter bank is not necessarily the most well suited. As we shall see in section VIII, the uniform DFT filter bank is not the most suitable choice in maximally decimated QMF bank applications. This is because perfect cancelation of aliasing with such systems typically requires synthesis filters of very high order. Moreover, perfect-reconstruction $M$-band QMF banks seldom have analysis filters satisfying the uniform shift property $H_{k}(z)=H_{0}\left(z W^{k}\right)$.

\section{Special Types of Digital Filters and Filter Banks}

A number of multirate applications benefit from the use of special types of systems such as half-band filters, $M$ th 
band filters, and power-complementary filter-banks. We shall now review these systems and indicate some applications.

\section{A. Half-band Filters, Mth Band Filters, and Nyquist Filters}

In most applications, these are linear-phase FIR filters. The impulse response $h(n)$ is assumed to be Hermitian-symmetric with respect to $n=0$ (i.e., $H(z)=\Sigma_{n=-\kappa}^{K} h(n) z^{-n}$ with $\left.h(n)=h^{*}(-n)\right)$, so that these are, in fact, zero-phase filters.

A half-band filter [35], [16, page 140] by definition, is a zerophase filter whose impulse response satisfies

$$
h(2 n)= \begin{cases}\mathrm{c} & n=0 \\ 0 & n \neq 0\end{cases}
$$

where $c$ is usually taken to be 0.5 . In other words, the evennumbered samples of $h(n)$, except $h(0)$, are equal to zero. If the transfer function $H(z)$ is written in the form of (7), we see immediately that the polyphase component $E_{0}(z)$ is a constant, i.e., $E_{0}(z)=$ c. Thus

$$
H(z)=c+z^{-1} E_{1}\left(z^{2}\right)
$$

from which we obtain

$$
H(\mathrm{z})+H(-\mathrm{z})=2 \mathrm{c} .
$$

Notice that with $z=\mathrm{e}^{j \omega}, H(-\mathrm{z})=H\left(\mathrm{e}^{j(\omega-\pi)}\right)$, which, for realcoefficient filters, reduces to $H\left(\mathrm{e}^{j(\pi-\omega)}\right)$. Thus, for a real-coefficient zero-phase FIR filter satisfying (16), we have

$$
H\left(\mathrm{e}^{j \omega}\right)+H\left(\mathrm{e}^{j(\pi-\omega)}\right)=1
$$

assuming $c$ is normalized to 0.5 . This shows that $H\left(e^{j(\pi / 2-\theta\}}\right)$ and $H\left(e^{j(\pi / 2+\theta)}\right)$ add up to unity for all $\theta$. In other words, we have a symmetry with respect to the half-band frequency $\pi / 2$, justifying the name "half-band filters." Fig. 22 demonstrates the effect of this symmetry for a low-pass

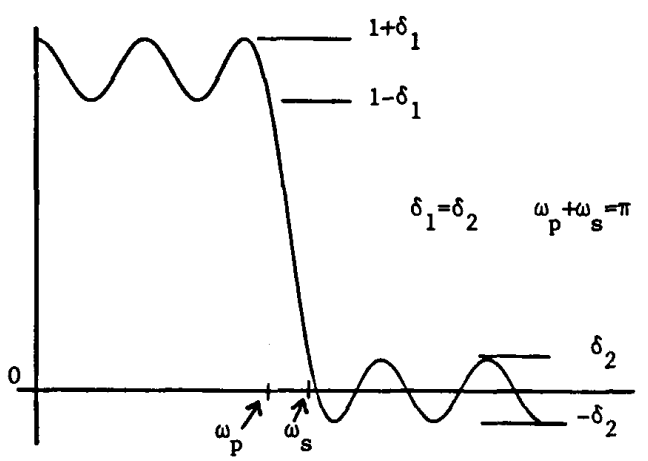

Fig. 22. Response of FIR half-band filter.

filter: the peak errors $\delta_{1}$ and $\delta_{2}$ are equal and the band edges $\omega_{p}$ and $\omega_{s}$ are equally distant from $\pi / 2$. These filters are, in particular, suitable as low-pass filters with cutoff $\omega_{c}$ at $\pi / 2$. The importance of half-band filters can be more thoroughly appreciated in section VI-B (see also [4] and [16, page 143]). Special tricks for the design of half-band filters can be found in [16, page 141] and in [36].

With $H(z)=\Sigma_{n=-K}^{K} h(n) z^{-n}$, it is clear that we can assume $K$ to be odd (otherwise $h(K)=h(-K)=0$, in view of (16), anyway). If we now define a causal version of $H(z)$ to be $H_{c}(z)$ $=z^{-\kappa} H(z)$, then the relation (18) translates to the well-known relation [10] $H_{c}(z)-H_{c}(-z)=z^{-k}$.

An $M$ th band filter [35] is a logical extension of a half-band filter. We shall define it as a zero-phase FIR filter with impulse response satisfying

$$
h(M n)= \begin{cases}c & n=0 \\ 0 & n \neq 0 .\end{cases}
$$

The constant $c$ is usually taken to be $1 / M$. The property of (20) is also called the Nyquist property, for historical reasons [37]. Representing $H(z)$ in the polyphase form of (10), it is clear that the component $E_{0}(z)$ is equal to the constant c. In other words, we can write $H(z)$ as

$$
\begin{aligned}
H(z)= & c+z^{-1} E_{1}\left(z^{M}\right)+z^{-2} E_{2}\left(z^{M}\right) \\
& +\cdots+z^{-(M-1)} E_{M-1}\left(z^{M}\right) .
\end{aligned}
$$

Naturally we expect such a filter to satisfy a logical extension of the property in (18). For this, consider the relation in (12), which holds for any $H(z)$. With $E_{0}(z)$ equal to the constant $c$, the summation on the LHS of (12) reduces to a constant, i.e.,

$$
\sum_{k=0}^{M-1} H\left(z W^{k}\right)=M c \quad \text { for all } z
$$

Note that any one of (20), (21), and (22) implies the other two. Clearly (18) is a special case of (22), with $M=2$. The significance of (22) is that the $M$ uniformly shifted frequency responses $H\left(e^{j(\omega-2 \pi k / M)}\right)$ add up to a contant for all $\omega$, if $H(z)$ is an $M$ th band filter).

Standard techniques for the design of FIR Mth band filters can be found in [35]. In most applications (such as in interpolation filter design and multilevel filter design (section (X-D)), the $M$ th band filter is required to be a linearphase low-pass filter with cutoff $\pi / M$. The simplest design procedure that guarantees the $M$ th band property is the well-known windowing technique [16], [17], in which the coefficients of $h(n)$ are obtained as

$$
h(n)=\frac{\sin (\pi n / M)}{\pi n} w(n)
$$

where $w(n)$ is a finite-duration sequence called the window function. If $w(n)$ is taken as the Kaiser window, then any desired stopband attenuation and transition bandwidth for $H(z)$ can be obtained by choice of the window length and a window parameter called $\beta$ [16, page 68]. The advantage of the window method is that the condition (20) is automatically satisfied, because $[\sin (\pi n / M)] / \pi n$ is zero whenever $n$ is a nonzero multiple of $M$.

It is well known that window methods do not result in filters that are optimal in any way. An alternative procedure to design $M$ th band filters that are optimal in the leastsquares sense is provided by the eigenfilter approach, the details of which can be found in [38, section V]. Finally, design procedures which seek to obtain approximately equiripple (i.e., optimal in the minimax sense) FIR $M$ th band filters can be found in [35].

For the case of arbitrary-phase FIR and IIR filters, one can still define the $M$ th band property by requiring that one out of the $M$ polyphase components $E_{k}(z)$ be constant. IIR $M$ th band filters of this type can be found in [39]. 


\section{B. Complementary Transfer Functions}

A set of transfer functions $\left[H_{0}(z), H_{1}(z), \cdots, H_{M-1}(z)\right]$ is said to be strictly complementary (SC) if they add up to a delay, i.e.

$$
\sum_{k=0}^{M-1} H_{k}(z)=c z^{-n_{0}}, \quad c \neq 0 .
$$

An example of this is the set $H_{k}(z)=H\left(z W^{k}\right), 0 \leq k \leq M-$ 1 , where $H(z)$ is an $M$ th band filter (see (22)). If we split a signal $x(n)$ into $M$ subband signals using such a set of filters (Fig. 10(a)), then the subband signals can simply be added to get back the original signal with no distortion except for a delay.

A set of $M$ transfer functions is said to be power-complementary $(\mathrm{PC})$ if

$$
\sum_{k=0}^{M-1}\left|H_{k}\left(e^{j \omega}\right)\right|^{2}=c \quad \text { for all } z,
$$

where $c \neq 0$ is constant. This property is equivalent to $\Sigma_{k=0}^{M-1} \tilde{H}_{k}(z) H_{k}(z)=c$ for all $z$, by analytic continuation. Such a property is useful in analysis/synthesis systems (i.e., in systems where Fig. 10(b) is cascaded to Fig. 10(a)). If the synthesis filters are chosen as $F_{k}(z)=\bar{H}_{k}(z)$, then we have $\hat{x}(n)$ $=c x(n)$, which implies perfect recovery of $x(n)$. In practice, the noncausality of $\tilde{H}_{k}(z)$ is avoided by insertion of a delay $z^{-n_{0}}$ so that $\hat{x}(n)=c x\left(n-n_{0}\right)$.

A set of transfer functions is said to be all-pass complementary $(A C)$ if

$$
\sum_{k=0}^{M-1} H_{k}(z)=A(z)
$$

where $A(z)$ is all-pass. If such a set is used in Fig. 10(a), and if the subband signals $x_{k}(n)$ are recombined by adding, the result $\hat{X}(n)$ satisfies $|\hat{X}(z)|=\left|X\left(e^{j \omega}\right)\right|$, so that there is no amplitude distortion in the reconstructed signal.

A set of transfer functions is said to be doubly complementary (DC) if it is AC and PC. There are several applications of this (including digital audio; see the following).

A pair of FIR transfer functions $\left[H_{0}(z), H_{1}(z)\right]$ is said to be Euclidean complementary (EC) if the polynomials $H_{0}(z)$ and $H_{1}(z)$ are relatively prime (i.e., do not share a common factor of the form $\left(1-\alpha z^{-1}\right)$ with $0<|\alpha|<\infty$. It is well known (Euclid's theorem [33, page 175]) that if $H_{0}(z)$ and $H_{1}(z)$ are relatively prime, then there exist polynomials $F_{0}(z)$ and $F_{1}(z)$ such that

$$
H_{0}(z) F_{0}(z)+H_{1}(z) F_{1}(z)=c, \quad c \neq 0 .
$$

This means that we can combine the outputs of $H_{0}(z)$ and $H_{1}(z)$ to reproduce $x(n)$ with no delay, even when the filters $H_{0}(z), H_{1}(z), F_{0}(z), F_{1}(z)$ are causal FIR! Here is a simple example which helps explain this surprising result: let $H_{0}(z)=$ $1+z^{-1}$ and $H_{1}(z)=1-z^{-1}$. Then the choice $F_{0}(z)=F_{1}(z)$ $=0.5$ results in $\hat{x}(n)=x(n)$. Given the relatively prime pair $\left[H_{0}(z), H_{1}(z)\right]$, there exists a unique pair $\left[F_{0}(z), F_{1}(z)\right]$ (up to a scale factor) of lowest degree which can be solved using Euclid's algorithm.

More generally, a set of $M$ FIR transfer functions $\left[H_{0}(z)\right.$, $\left.H_{1}(z), \cdots, H_{M-1}(z)\right]$ is said to be EC if there is no factor (1 $\left.-\alpha z^{-1}\right)$ with $0<|\alpha|<\infty$ common to all of these. Under this condition, there exists a set of $M$ FIR filters $\left[F_{0}(z), F_{1}(z)\right.$, $\left.\cdots, F_{M-1}(z)\right]$ such that $\Sigma_{k=0}^{M-1} H_{k}(z) F_{k}(z)=1$. In particular, if all $H_{k}(z)$ are causal, then so are all $F_{k}(z)$.
A warning is appropriate in this context. Suppose the relatively prime polynomials $H_{0}(z)$ and $H_{1}(z)$ are causal low-pass and high-pass filters with $\left|H_{0}\left(e^{j \omega}\right)\right|,\left|H_{1}\left(e^{j \omega}\right)\right| \leq 1$. Then the unique lowest degree pair $\left[F_{0}(z), F_{1}(z)\right]$ turns out to be highpass and low-pass, respectively (try it!). So with $F_{0}(z), F_{1}(z)$ normalized so that $\left|F_{0}\left(\mathrm{e}^{j \omega}\right)\right|,\left|F_{1}\left(\mathrm{e}^{j \omega}\right)\right| \leq 1$, the constant $\mathrm{c}$ in (26) can be very small. This means that quantization errors in the design and implementation of the filters can dominate $c$, rendering this scheme impractical.

\section{Relation Between Mth Band Filters and Power- Complementary Filters}

Consider a transfer function $H(z)$ represented in the $M$-component polyphase form (10). Define the new transfer function $G(z)=\tilde{H}(z) H(z)$. Then the set $\left[E_{0}(z), E_{1}(z), \cdots\right.$, $\left.E_{M-1}(z)\right]$ is power complementary if and only if $G(z)$ is an $M$ th band filter. A proof of this can be found in Appendix B.

\section{Design of Special Transfer Functions}

Since the design procedures are well documented in the literature, we shall be content with mentioning a few pointers here. The design of linear-phase FIR half-band filters is discussed in [35] and [16, page 140]. The design of FIR linearphase $M$ th band and Nyquist filters is described in [35], [36], and [38]. As mentioned in section V-A, the simplest approach for this is based on the window method.

Given an FIR filter $H_{0}(z)$ with $\left|H_{0}\left(e^{j \omega}\right)\right| \leq 1$, it is easy to find an FIR filter $H_{1}(z)$ such that $\left[H_{0}(z), H_{1}(z)\right]$ is PC. For this, note that $\mathrm{PC}$ property is equivalent to

$$
\left|H_{1}\left(\mathrm{e}^{j \omega}\right)\right|^{2}=1-\left|H_{0}\left(\mathrm{e}^{j \omega}\right)\right|^{2} .
$$

In other words, $H_{1}(z)$ is a spectral factor of the quantity 1 - $\left|H_{0}\left(\mathrm{e}^{j \omega}\right)\right|^{2}$. The coefficients of such a spectral factor can be computed as follows: compute the zeros of the polynomial $1-\tilde{H}_{0}(z) H_{0}(z)$ (which is equal to $\tilde{H}_{1}(z) H_{1}(z)$ ), and denote these as $z_{1}, z_{2}, \cdots, z_{2 K}$, where $K$ is the order of $H_{0}(z)$. These zeros occur in reciprocal conjugate pairs, i.e., if $\alpha$ is a zero then so is $1 / \alpha^{*}$. Construct $H_{1}(z)$ by arbitrarily picking one out of every such pair (this arbitrariness implies that the spectral factor need not be unique). Since all the zeros of $H_{1}(z)$ are determined in this way, $H_{1}(z)$ is determined up to a scale factor. Methods for computing $H_{1}(z)$ without explicitly finding the zeros of $1-\bar{H}_{0}(z) H_{0}(z)$ are described in [40] and [41]. The method in [40] works even if there are zeros on the unit circle, whereas the method reported in [41] works only if there are no unit-circle zeros.

Finally, IIR filters with doubly complementary properties are elaborated in [42] and [43]. Some more elaboration on a special class of IIR transfer functions with the double complementary property can also be found in section VI-A of this paper.

\section{E. Applications of Special Transfer Functions}

From the preceding discussions it is clear that $M$ th band filters and complementary filters have applications in the exact reconstruction of a signal $x(n)$ after it has been split into $M$ subbands (provided the subband signals are not decimated; decimation would cause aliasing error, which is a major issue discussed in section VI). A second application of $M$ th band filters is that they are ideally suited as interpolation filters. Recall that the input to the interpo- 
lation filter in Fig. $7($ b) has $M-1$ zeros between adjacent nonzero samples (with $L=M$ ) and that the purpose of $H(z)$ is to fill these zero-valued samples with a weighted average of nonzero samples. Clearly, it is desirable to communicate the original nonzero samples of $x_{1}(n)$ without change. This is accomplished [37] if the impulse response $h(n)$ satisfies (20).

Complementary Filters in Digital Audio: The loudspeaker system in most audio equipment typically has different subspeakers for different frequency ranges; e.g., the tweeter (high frequency) and woofer (low frequency). In a digital audio system it is desirable to split the signal $y(n)$ (before $\mathrm{D} / \mathrm{A}$ conversion) into the low-pass signal $y_{0}(n)$ and high-pass signal $y_{1}(n)$ using an analysis bank $\left[H_{0}(z), H_{1}(z)\right]$. The analysis bank is more commonly called a "crossover network" in the audio industry. (See [44] and [45] for analog-filter examples of this type.) These subband signals can then be D/A converted and fed into the speakers. Assuming that the loudspeaker introduces negligible distortion (which, in general, is not true), the human ear eventually perceives an analog version of $y_{0}(n)+y_{1}(n)$. In the transform domain, this is $\left[H_{0}(z)+H_{1}(z)\right] Y(z)$. To avoid any distortion in the reconstruction, it is desirable to design $H_{0}(z)$ and $H_{1}(z)$ to be a strictly complementary pair. This requires the use of linearphase FIR half-band filters, which are more expensive than IIR filters. With IIR filters it is possible to force the all-pass complementarity. This means that $\left[H_{0}(z)+H_{1}(z)\right] Y(z)$ represents $Y(z)$ faithfully except for phase distortion. If necessary, phase distortion can be equalized using an all-pass filter (section VI-A).

It is very desirable to design $H_{0}(z)$ and $H_{1}(z)$ to be good low-pass and high-pass filters so that the speakers are not damaged by out-of-band energy. Notice, however, that if $H_{0}(z)$ is a good low-pass filter and if $\left[H_{0}(z), H_{1}(z)\right]$ is $A C$, this does not mean $H_{1}(z)$ is a good high-pass filter. This is because the responses $H_{0}\left(e^{j \omega}\right)$ and $H_{1}\left(e^{j \omega}\right)$ are, in general, complex. For example, it is possible at some frequency to have $H_{0}\left(\mathrm{e}^{j \omega}\right)$ $=\mathrm{e}^{j \pi / 3}$ and $H_{1}\left(\mathrm{e}^{j \omega}\right)=\mathrm{e}^{-i \pi / 3}$ so that the sum is $2 \cos (\pi / 3)=$ 1, consistent with the all-pass requirement of $H_{0}(z)+H_{1}(z)$ For this reason a doubly complementary pair $\left[H_{0}(z), H_{1}(z)\right]$ is most suitable: the PC property ensures that $H_{1}(z)$ is a good high-pass filter (if $H_{0}(z)$ is low-pass) and the $A C$ property eliminates amplitude distortion. Such IIR filters can be implemented much more efficiently than FIR filters, as elaborated in [42], [43], and in section VI-A. For systems with several subband speakers, an $M$-band AC filter bank can be used; see [39] for the design of such filters.

More applications of special transfer functions are scattered throughout this paper in various forms.

\section{The Two-Channel QMF Bank}

Consider the filter bank system of Fig. 13, which is a maximally decimated analysis/synthesis system (or a QMF bank) [4], [46]-[50]. The applications of this system were outlined in greater detail in section III. The QMF bank is an LTV system (because of decimators and interpolators). In this section we shall study the aliasing and imaging effects created by the decimators and interpolators, emphasizing the $M=$ 2 case. The filters $H_{0}(z)$ and $H_{1}(z)$ are low-pass and high-pass, respectively (Fig. 12(b)). The overlapping responses of Fig. 12(b) ensures that no portion of $X\left(e^{j \omega}\right)$ is severely attenuated. However, this overlap also means that the filters do not bandlimit the subband signals $x_{0}(n)$ and $x_{1}(n)$ to a sufficient extent, which results in aliasing when $x_{0}(n)$ and $x_{1}(n)$ are decimated. We should choose the synthesis filters $F_{0}(z)$ and $F_{1}(z)$ to cancel this aliasing, as is explained next.

\section{A. Analysis and Elimination of Errors in the QMF Bank}

Since aliasing is permitted, none of the complementary filters described in section $V$ can be used for perfect reconstruction without canceling this aliasing.

Consider a typical input $x(n)$ with $X\left(\mathrm{e}^{j \omega}\right)$ as in Fig. 23(a). The signal $x_{0}(n)$ has transform as in Fig. 23(b). From (3b) we deduce that $V_{0}\left(\mathrm{e}^{j \omega}\right)=\left[X_{0}\left(\mathrm{e}^{j \omega / 2}\right)+X_{0}\left(-\mathrm{e}^{j \omega / 2}\right)\right] / 2$, which describes the aliasing effect in the top channel. From (3a) we see that $Y_{0}\left(e^{j \omega}\right)=V_{0}\left(e^{2 j \omega}\right)$, which describes the imaging effect. In a similar way, we can trace through the bottom channel in Fig. 13. The transforms of the various signals in Fig. 13 are therefore as shown in Fig. 23.

Our aim is to combine the signals $y_{0}(n)$ and $y_{1}(n)$ with the help of the filters $F_{0}(z)$ and $F_{1}(z)$ such that aliasing is canceled. The shaded areas in Figs. 23(d) and (g) represent the two alias components. It is evident that the filters $F_{0}(z)$ and $F_{1}(z)$ should be low-pass and high-pass, respectively, so that the major part of the aliasing is removed. After this, the only alias components of considerable significance are around $\omega=\pi / 2$ and $\omega=3 \pi / 2$, as shown in Figs. 23(h) and 23(i). These nonzero components remain because the analysis filters overlap around $\pi / 2$. (Fig. 12(b)). Our aim is to cancel these two nonzero components.

By using (3a) and (3b) we see that the general relation between $\hat{X}(z)$ and $X(z)$ is given by

$$
\begin{aligned}
\hat{X}(z)= & \frac{1}{2}\left[H_{0}(z) F_{0}(z)+H_{1}(z) F_{1}(z)\right] X(z) \\
& +\frac{1}{2}\left[H_{0}(-z) F_{0}(z)+H_{1}(-z) F_{1}(z)\right] X(-z) .
\end{aligned}
$$

The term containing $X(-z)$, which is the alias component, vanishes if we choose

$$
F_{0}(z)=H_{1}(-z), \quad F_{1}(z)=-H_{0}(-z) .
$$

With aliasing canceled in this manner we have

$$
\hat{X}(z)=T(z) X(z) .
$$

The LTV system of Fig. 13 thus becomes an LTI system with transfer function

$$
T(z)=\frac{\hat{X}(z)}{X(z)}=\frac{1}{2}\left[H_{0}(z) F_{0}(z)+H_{1}(z) F_{1}(z)\right]
$$

which represents the distortion caused by the QMF bank. We shall call $T(z)$ the overall transfer function (or the distortion transfer function).

In general, because of $T(z), \hat{x}(n)$ suffers from amplitude distortion (if $\left\{T\left(e^{j \omega}\right) \mid\right.$ is not constant for all $\omega$ ) and from phase distortion (if $T(z)$ does not have linear phase). To eliminate amplitude distortion it is necessary to force $T(z)$ to be allpass, whereas to eliminate phase distortion, we have to force $T(z)$ to be FIR with linear phase. Both of these distortions are eliminated if $T(z)$ is a pure delay, i.e.,

$$
T(z)=c z^{-n_{0}}
$$

or, equivalently,

$$
\hat{x}(n)=c x\left(n-n_{0}\right) .
$$




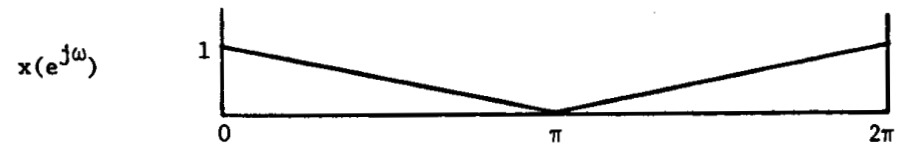

(a)

$x_{0}\left(e^{j \omega}\right)$

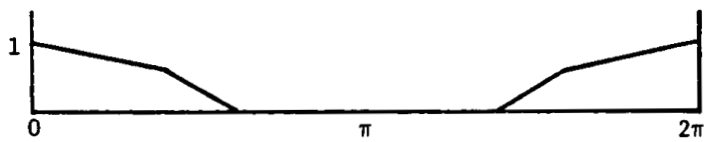

(b)

$v_{0}\left(e^{j \omega}\right)$

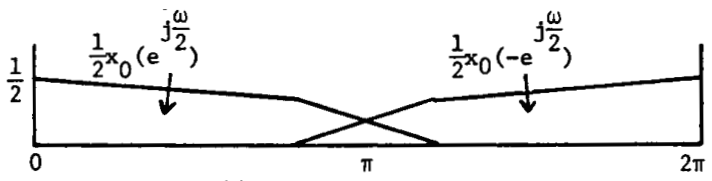

(c)

$y_{0}\left(e^{j \omega}\right)$

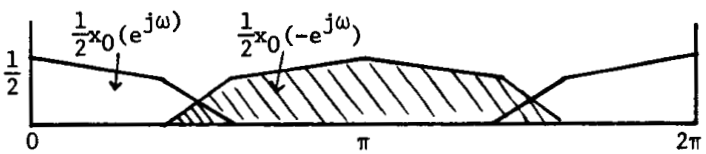

(d)

$x_{1}\left(e^{j \omega}\right)$

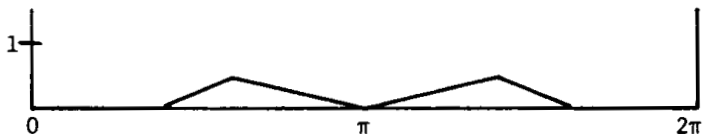

(e)

$v_{1}\left(e^{j \omega}\right)$

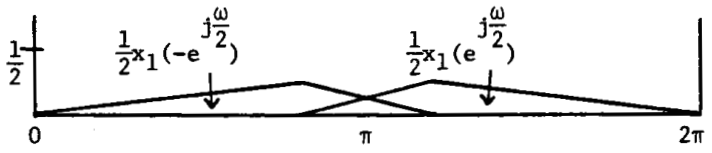

(f)

$y_{1}\left(e^{j \omega}\right)$

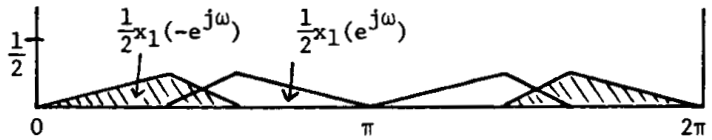

(g)

Allas-term at Output of $F_{0}(z)$

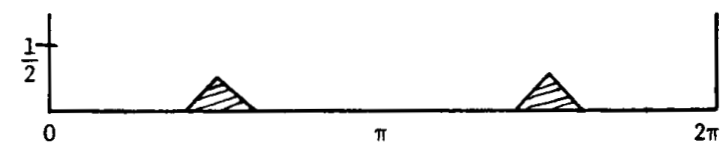

(h)

Alias-term at Output

of $F_{1}(z)$

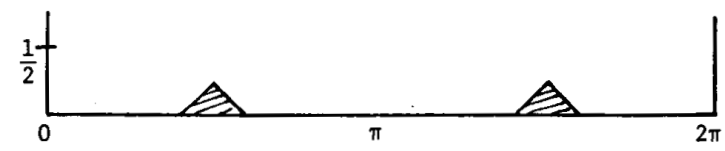

(i)

Fig. 23. Illustration of various Fourier transforms in two-channel QMF bank. Here horizontal axis represents $\omega$. (a) Typical input. (b) Transform. (c) Aliasing effect. (d) Imaging effect. (e) Using $x_{1}$. (f) Using $v_{1}$. (g) Using $y_{1}$. (h) Alias-term at output of $F_{0}(z)$. (i) Alias-term at output of $F_{7}(z)$

Summarizing, the reconstructed signal $\hat{x}(n)$ in Fig. 13 suffers from three distortions: aliasing distortion (ALD), amplitude distortion (AMD), and phase distortion (PHD). In addition, we have the nonlinear coding error created by the encoding (or quantization) of the decimated signals. Since coding error cannot be eliminated, we shall not discuss it further. On the other hand, we shall see that the first three errors can be completely eliminated at finite cost by careful choice of the filters $H_{k}(z)$ and $F_{k}(z)$. Such systems which are alias-free and satisfy (31) are called perfect-reconstruction (PR) systems.

Elimination of Phase Distortion: For any pair of analysis 
filters, the choice of (28) of the synthesis filters eliminates ALD. We then have

$$
T(z)=\frac{1}{2}\left[H_{0}(z) H_{1}(-z)-H_{1}(z) H_{0}(-z)\right] .
$$

It is easy to force $T(z)$ to be an FIR linear-phase filter simply by forcing $H_{0}(z)$ and $H_{1}(z)$ to be linear-phase FIR.

In the earliest known QMF banks [4], [46], the analysis filters were related to each other as follows:

$$
\begin{aligned}
& H_{1}(z)=H_{0}(-z) \\
& F_{0}(z)=H_{0}(z), \quad F_{1}(z)=-H_{1}(z)=-H_{0}(-z) .
\end{aligned}
$$

The choice of (34) ensures that $H_{1}(z)$ is a high-pass filter if $H_{0}(z)$ is low pass. The choice of (35) is equivalent to that of (28) under the condition of (34), thus eliminating aliasing. We then have

$$
T(z)=\frac{1}{2}\left[H_{0}^{2}(z)-H_{0}^{2}(-z)\right]=2 z^{-1} E_{0}\left(z^{2}\right) E_{1}\left(z^{2}\right)
$$

which automatically has linear phase as long as $H_{0}(z)$ is chosen to have linear phase (the RHS of (36) is obtained by writ- ing $H_{0}(z)$ as in (7)). With ALD and PHD eliminated in this way, we still are left with AMD. To demonstrate this, Fig. 24(a) shows three linear-phase designs of $H_{0}(z)$. The corresponding plots of $\left|T\left(\mathrm{e}^{j \omega}\right)\right|$ are shown in Fig. 24(b), demonstrating the existence of AMD (because the plot is not constant for all $\omega$. If there is too much overlap between the filters $H_{0}(z)$ and $H_{1}(z)$ (curve 1), then there is peaking in $\left|T\left(\mathrm{e}^{j \omega}\right)\right|$, around $\omega=\pi / 2$. If the overlap is too small (curve 2), then $\left|T\left(\mathrm{e}^{j \omega}\right)\right|$ dips around $\pi / 2$. An optimal overlap based on trial-and-error results in curve 3 , which appears to be fairly good (though not very exciting).

From (36) we see that the system is PR if and only if $E_{0}(z) E_{1}(z)$ is a pure delay. Since $H_{0}(z)$ is restricted to be FIR, this is possible if and only if $E_{0}(z)$ and $E_{1}(z)$ are delays, which means $H_{0}(z)$ must have the form

$$
H_{0}(z)=c_{0} z^{-2 n_{0}}+c_{1} z^{-\left(2 n_{1}+1\right)} .
$$

Summarizing, a two-channel FIR QMF bank with filters restricted as in (34) can have PR property if and only if $H_{0}(z)$ is a trivial transfer function of the form (37).

In practice, frequency responses are required to be more

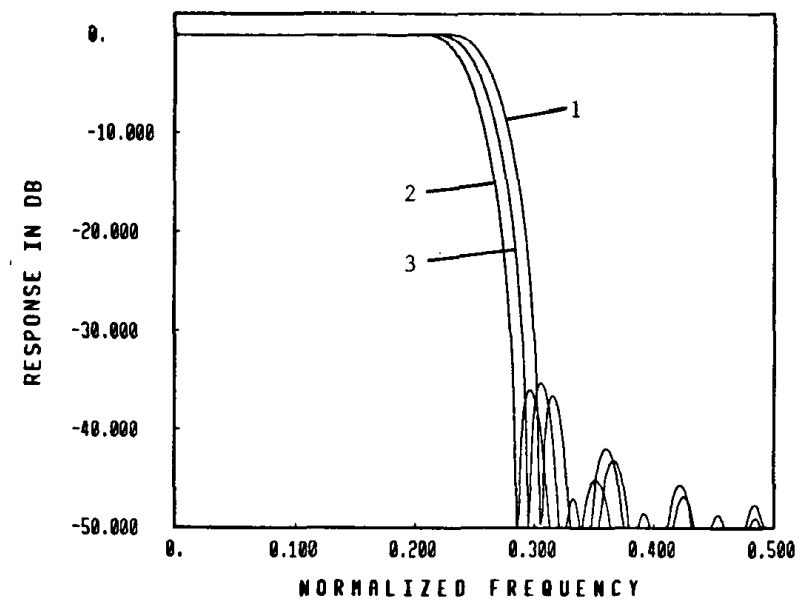

(a)

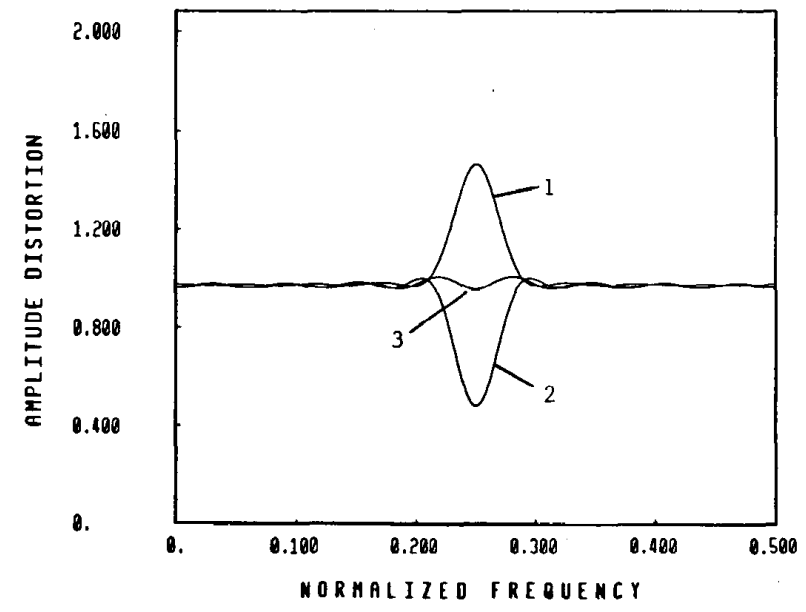

(b)

Fig. 24. Three design examples for FIR QMF bank, demonstrating amplitude distortion. (a) Response of analysis filters $H_{0}(z)$. (b) Corresponding amplitude distortions. 
sophisticated than (37). So, under the constraint of (34), perfect reconstruction is not possible. After eliminating ALD and PHD, it is, however, possible to minimize AMD systematically. To be more specific, one optimizes the coefficients of $H_{0}(z)$ such that the function $\left|T\left(\mathrm{e}^{j \omega}\right)\right|$ is made as flat as possible, while at the same time minimizing the stopband energy of $H_{0}(z)$. Thus an objective function of the form

$$
\begin{aligned}
& \alpha \int_{\omega S}^{\pi}\left|H_{0}\left(\mathrm{e}^{j \omega}\right)\right|^{2} d \omega+(1-\alpha) \int_{0}^{\pi / 2}\left[1-\left|T\left(\mathrm{e}^{j \omega}\right)\right|^{2}\right]^{2} d \omega, \\
& 0<\alpha<1
\end{aligned}
$$

can be minimized by optimizing the coefficients of $H_{0}(z)$. The second integral in (38) goes only up to $\pi / 2$ because $\left|T\left(e^{j \omega}\right)\right|$ has period $\pi$ rather than $2 \pi$ (see (36)). The coefficient $\alpha$ is used to control the tradeoff between the stopband energy of $H_{0}(z)$ and the flatness of $\left|T\left(e^{j \omega}\right)\right|$. Such optimization has been done by Johnston [48], and by Jain and Crochiere [49]. Several tables of optimal filter coefficients can be found in [4] and [48].

An efficient implementation of the preceding two-channel QMF bank can be obtained using the polyphase decomposition. In view of (34), the analysis bank can be drawn as in Fig. 19. The decimators which follow this can be moved to the left of $E_{0}\left(z^{2}\right)$ and $E_{1}\left(z^{2}\right)$, so that the entire analysis bank requires only about N/2 MPUs and N/2 APUs. And in view of the relation of (35), the synthesis bank can be implemented with equal efficiency. Fig. 25 shows the complete

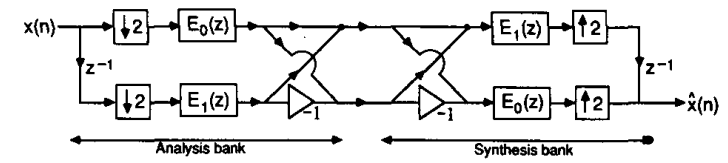

Fig. 25. Complete two-band QMF bank with $H_{1}(z)=H_{0}(-z)$, $F_{0}(z)=H_{0}(z), F_{1}(z)=-H_{1}(z)$.

implementation using the polyphase framework. Notice that the complete system in Fig. 25 requires a total of only about $N$ MPUs and NAPUs, where $N$ is the length of the low-pass filter $H_{0}(z)$.

Recall that in the preceding method $H_{0}(z)$ is required to have linear phase if $T(z)$ must have linear phase. A caution about the choice of $N$ is necessary here: $N$ should be chosen to be even. An odd value of $N$ results in $T\left(e^{j \omega}\right)$, which is necessarily zero at $\omega= \pm \pi / 2$. For proof, see [4], [47] or [50, page 7]. A different proof is obtained by looking at the RHS of (36): if $N$ is odd, then $E_{0}(z)$ and $E_{1}(z)$ are linear-phase filters, and one of these necessarily has odd order, resulting in a zero at $\omega=\pi[16$, page 73$]$. This translates into a zero of (36) at $\omega= \pm \pi / 2$.

Elimination of Amplitude Distortion: The choice of filters as in (34) and (35), with $H_{0}(z)$ chosen as linear-phase FIR, eliminated ALD and PHD completely, and AMD was then minimized by optimization. A complementary approach would be to choose the filters to eliminate ALD and AMD completely, and minimize PHD. This can indeed be accomplished as follows. Again choose the filters as in (34) and (35) so that (28) is satisfied, resulting in ALD elimination. Now we have $T(z)$ as in (36), which we wish to force to be all-pass. From the RHS of (36) we see that this can be done by constraining the polyphase components $E_{0}(z)$ and $E_{1}(z)$ to be IIR all-pass! This is precisely equivalent to constraining the analysis filter $H_{0}(z)$ to be of the form

$$
H_{0}(z)=\frac{1}{2}\left[A_{0}(z)+A_{1}(z)\right]
$$

where $A_{0}(z)$ and $A_{1}(z)$ are all-pass filters of the form

$$
A_{0}(z)=a_{0}\left(z^{2}\right), \quad A_{1}(z)=z^{-1} a_{1}\left(z^{2}\right) .
$$

The relevant question now is this: is it possible to obtain good low-pass filters under the constraint that the transfer function be of the form of (39a)? The answer, fortunately, is yes!

To understand this, recall that the frequency response of the all-pass functions can be expressed as $A_{0}\left(\mathrm{e}^{j \omega}\right)=\mathrm{e}^{j \phi_{0}(\omega)}$ and $A_{1}\left(e^{j \omega}\right)=e^{j \phi_{1}(\omega)}$ so that (39a) takes the form

$$
H_{0}\left(\mathrm{e}^{j \omega}\right)=\frac{\mathrm{e}^{j \phi_{0}(\omega)}+\mathrm{e}^{j \phi_{1}(\omega)}}{2} .
$$

If the all-pass functions are designed such that the phase responses $\phi_{0}(\omega)$ and $\phi_{1}(\omega)$ are aligned in the passband and differ by $\pi$ in the stopband, $\left|H_{0}\left(e^{j \omega}\right)\right|$ automatically comes out to be a good low-pass response! In fact, it can be shown [42] that digital Butterworth, Chebyshev, and Elliptic filters are special cases of the form (39a).

The further constraint (39b) on the all-pass filters is necessary to ensure that $A_{0}(z) / 2$ and $A_{1}(z) / 2$ are the components $E_{0}\left(z^{2}\right)$ and $z^{-1} E_{1}\left(z^{2}\right)$, so that (36) will work out to be all-pass, thus eliminating AMD. We then have $T(z)=z^{-1} a_{0}\left(z^{2}\right) a_{1}\left(z^{2}\right)$ / 2. The phase distortion created by the nonlinear phase of $T\left(e^{j \omega}\right)$ can be reduced by all-pass equalization.

Design Procedure: Perhaps the simplest procedure to design filters of this form involves the recognition [42], [43] that digital elliptic filters (which are optimal in the minimax sense) automatically have the form of (39a). If the order of $H_{0}(z)$ is odd, the coefficients of $A_{0}(z)$ and $A_{1}(z)$ are real [42], and we shall assume it is so here. The only remaining step is to ensure that $A_{0}(z)$ and $A_{1}(z)$ have the forms $a_{0}\left(z^{2}\right)$ and $z^{-1} a_{1}\left(z^{2}\right)$, respectively. Now with $H_{0}(z)=\left[a_{0}\left(z^{2}\right)+z^{-1} a_{1}\left(z^{2}\right)\right] /$ 2 and $H_{1}(z)=H_{0}(-z)$, we can verify by direct substitution that $\left|H_{0}\left(e^{j \omega}\right)\right|^{2}+\left|H_{1}\left(e^{j \omega}\right)\right|^{2}=1$, i.e.,

$$
\left|H_{0}\left(e^{j \omega}\right)\right|^{2}+\left|H_{0}\left(e^{j(\pi-\omega)}\right)\right|^{2}=1 .
$$

In other words, $\left|H_{0}\left(e^{j \omega}\right)\right|^{2}$ has a symmetry, with respect to $\pi / 2$, analogous to Fig. 22.

The procedure would then be to design an odd-order elliptic filter $H_{0}(z)$ (of specified attenuation and transition width) with passband ripple $\delta_{1}$ and stopband ripple $\delta_{2}$ such that the aforementioned symmetry holds (i.e., $1-\left(1-2 \delta_{1}\right)^{2}$ $=\delta_{2}^{2}$ ). With all the filter specifications now available, a standard digital elliptic filter design procedure (such as the one in [9, pp. 125-127] followed by bilinear transformation) can be used to design $H_{0}(z)$.

The filter so designed is guaranteed to have the following form:

$$
H_{0}(z)=\frac{N(z)}{d\left(z^{2}\right)}
$$

because of the half-band symmetry, which guarantees that the poles of $H_{0}(z)$ are confined to the imaginary axis of the $z$ plane (see [43, Appendix] for formal proof). This ensures that $d(z)$ has the form

$$
d(z)=\prod_{k=1}^{r}\left(1+d_{k} z^{-1}\right)
$$


with

$$
0<d_{1}<d_{2}<\cdots<d_{r}<1 .
$$

The filters $a_{0}(z)$ and $a_{1}(z)$ are now identified [51] as

$$
a_{0}(z)=\prod_{k \text { odd }} \frac{d_{k}+z^{-1}}{1+d_{k} z^{-1}}, \quad a_{1}(z)=\prod_{k \text { even }} \frac{d_{k}+z^{-1}}{1+d_{k} z^{-1}} .
$$

Once the polyphase components $E_{0}(z)=a_{0}(z) / 2$ and $E_{1}(z)=$ $a_{1}(z) / 2$ are so determined, we can implement the complete QMF bank as in Fig. 25. The phase distortion of the QMF bank (caused by the nonlinearity of phase $\phi(\omega)$ of $T(z)=$ $\left.z^{-1} a_{0}\left(z^{2}\right) a_{1}\left(z^{2}\right) / 2\right)$, can be seen by plotting the group delay $\tau(\omega)=-d \phi(\omega) / d \omega$. The nonlinearity of $\phi(\omega)$ corresponds to a nonflat nature of $\tau(\omega)$. This is particularly severe near the band edge (i.e., around $\omega=\pi / 2$ ), and particularly so for filters with sharp transition bands and/or large attenuations.

Design Example: Assume that $H_{0}(z)$ is required to have a stopband attenuation of $40 \mathrm{~dB}$ and stopband edge $\omega_{s}=$ $0.62 \pi$. Because of the half-band symmetry, this implies a peak passband ripple $\delta_{1}=0.25 \times 10^{-4}$ and passband edge $\omega_{p}=0.38 \pi$. The elliptic low-pass design, based on [9], then results in $H_{0}(z)=\left[a_{0}\left(z^{2}\right)+z^{-1} a_{1}\left(z^{2}\right)\right] / 2$, where

$$
a_{0}(z)=\frac{0.211056+z^{-1}}{1+0.211056 z^{-1}}, \quad a_{1}(z)=\frac{0.685604+z^{-1}}{1+0.685604 z^{-1}} .
$$

Fig. 26(a) shows the magnitude response of $H_{0}(z)$. The group delay of the QMF bank (i.e., the group delay $\tau(\omega)$ of $T(z)=$ $\left.z^{-1} a_{0}\left(z^{2}\right) a_{1}\left(z^{2}\right)\right)$ is shown in Fig. 26(b). This exhibits a peak around $\pi / 2$ as expected. The group delay distortion (which is the difference between the largest and the smallest values of $\tau(\omega))$ is approximately equal to 14 samples.

A ninth-order all-pass equalizer of the form

$$
E(z)=\frac{-\beta+z^{-1}}{1-\beta z^{-1}} \prod_{k=1}^{4} \frac{R_{k}^{2}-\left(2 R_{k} \cos \theta_{k}\right) z^{-1}+z^{-2}}{1-\left(2 R_{k} \cos \theta_{k}\right) z^{-1}+R_{k}^{2} z^{-2}}
$$

is used to compensate for the group delay distortion of $a_{0}(z) a_{1}(z)$. This means that the reconstructed signal $\hat{x}(n)$ must be passed through $E\left(z^{2}\right)$ to get the equalized output. Fig. 26(b) also shows the group delay of the complete QMF bank with the equalizer. This group delay is fairly flat and exhibits

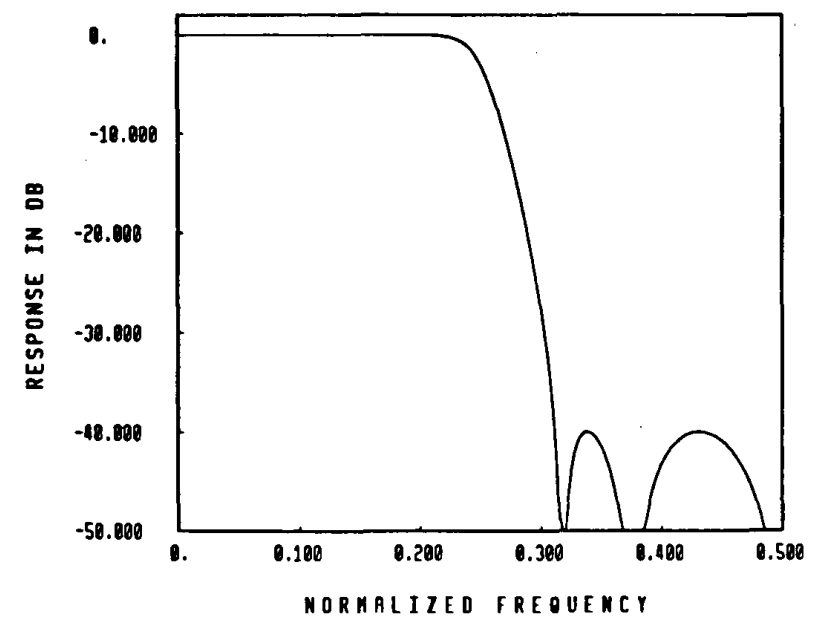

(a)

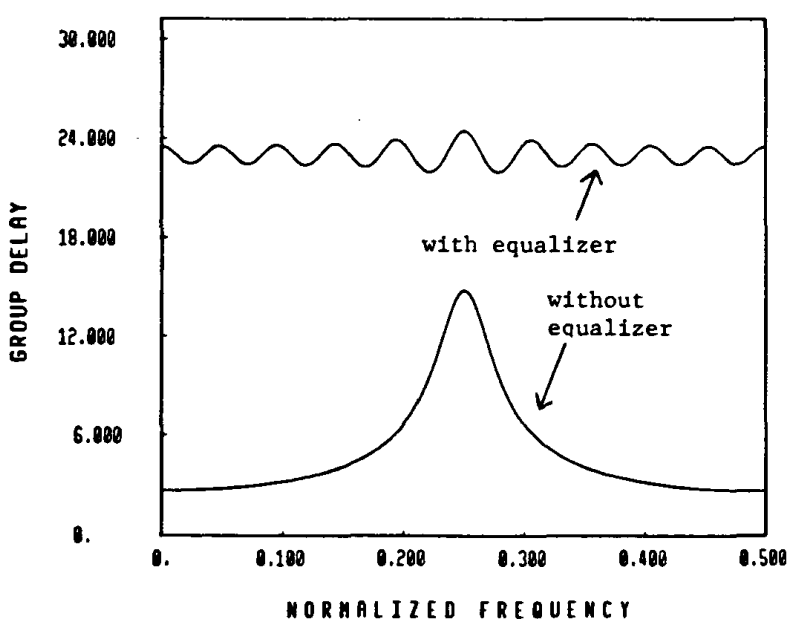

(b)

Fig. 26. Design example for IIR all-pass-based QMF bank. (a) Magnitude response of $H_{0}(\mathrm{z})$.

(b) Group delay of complete QMF bank with and without equalizer. 
a distortion of only about 2.5 samples. Note that the overall group delay of the equalized QMF bank is approximately equal to 23 samples. The coefficients $\beta, R_{k}$, and $\theta_{k}$ were obtained by minimizing an objective function of the form

$$
\int_{0}^{\pi}\left[\tau_{1}(\omega)+\tau_{2}(\omega)-c\right]^{2} d \omega
$$

where $\tau_{1}(\omega)$ is the group delay of $a_{0}(z) a_{1}(z)$ and $\tau_{2}(\omega)$ is the group delay of $E(z)$. The quantity $c$ of this is an unknown constant, and is among the parameters being optimized The equalizer parameters found in this matter turned out to be

$$
\begin{aligned}
& R_{1}=0.673364, \quad R_{2}=0.660524, \quad R(3)=0.662805, \\
& R(4)=0.666467, \\
& \theta_{1}=0.7791 \pi, \quad \theta_{2}=0.1896 \pi, \quad \theta_{3}=0.3808 \pi, \\
& \theta_{4}=0.5759 \pi,
\end{aligned}
$$

and $\beta=0.659$.

There exist a number of digital filter structures for implementing all-pass filters [52], [53] which have the following property: the transfer function remains (stable and) all-pass, despite quantization of the multipliers. In other words, the transfer function is structurally all-pass (i.e., all-pass despite multiplier values). This is very desirable: if we implement the QMF bank as in Fig. 25, with the functions $E_{0}(z)$ and $E_{1}(z)$ constrained to be structurally all-pass, then ALD and AMD are eliminated regardless of quantization of the structure coefficients.

See the end of section VI for a discussion of implementation complexity and for a comparison with other types of two-channel QMF banks of comparable performance.

\section{B. Two-channel Perfect Reconstruction QMF Banks}

Next, how can we eliminate all three distortions, viz., ALD, AMD, and PHD simultaneously? Such elimination would of course imply (31), i.e., perfect reconstruction. We can create a very simple example of a PR system by choosing the filters as follows:

$$
H_{0}(z)=1, \quad H_{1}(z)=z^{-1}, \quad F_{0}(z)=z^{-1}, \quad F_{1}(z)=1 .
$$

This system is shown in Fig. 27(a). By employing (27) we can verify that (29) is satisfied with $T(z)=z^{-1}$ so that $\hat{x}(n)=x(n$ -1). This can also be seen from Fig. 27(a) by noting that the analysis bank merely partitions the input samples into evenand odd-numbered subsets. The synthesis bank combines these subsets and puts the samples back in their original places (except for a delay $z^{-1}$ ).

Even though the filters (41) have trivial frequency responses, this example proves the existence of FIR PR QMF banks. We shall make use of Fig. 27(a) to construct nontrivial FIR PR QMF examples, where the filters $H_{0}(z)$ and $H_{1}(z)$ have good attenuation. We shall seek the help of the polyphase decomposition to make the connection between Fig. 27(a) and more nontrivial systems.

Polyphase Decomposition of the QMF Bank: We have already seen that a two-channel QMF bank can be drawn as in Fig. 25 when the filters are related by (34) and (35). We also saw that if the filters are so related, then perfect reconstruction is not possible unless the frequency response of

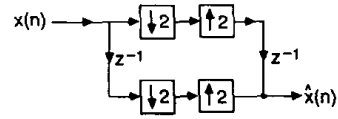

(a)

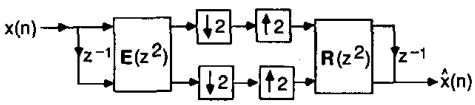

(b)

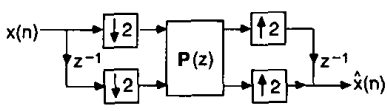

(c)

Fig. 27. Pertaining to design of perfect-reconstruction systems. (a) Simple perfect-reconstruction system. (b) Redrawing two-band QMF bank. (c) Here $\boldsymbol{P}(z)=\boldsymbol{R}(z) \boldsymbol{E}(z)$.

$H_{0}(z)$ is as in (37). Let us, therefore, not assume at the beginning any relationship between filters.

Suppose each of the analysis filters is written in the form of (7), i.e., $H_{k}(z)=E_{k 0}\left(z^{2}\right)+z^{-1} E_{k 1}\left(z^{2}\right), k=0$, 1. Similarly let each synthesis filter be written in the form (13), i.e., $F_{k}(z)=$ $z^{-1} R_{0 k}\left(z^{2}\right)+R_{1 k}\left(z^{2}\right)$. We can collect these equations neatly in matrix-form as follows:

$$
\left[\begin{array}{l}
H_{0}(z) \\
H_{1}(z)
\end{array}\right]=\left[\begin{array}{ll}
E_{00}\left(z^{2}\right) & E_{01}\left(z^{2}\right) \\
E_{10}\left(z^{2}\right) & E_{11}\left(z^{2}\right)
\end{array}\right]\left[\begin{array}{l}
1 \\
z^{-1}
\end{array}\right]
$$

and

$$
\left[F_{0}(z) \quad F_{1}(z)\right]=\left[\begin{array}{ll}
z^{-1} & 1
\end{array}\right]\left[\begin{array}{ll}
R_{00}\left(z^{2}\right) & R_{01}\left(z^{2}\right) \\
R_{10}\left(z^{2}\right) & R_{11}\left(z^{2}\right)
\end{array}\right] .
$$

With this, we can redraw Fig. 13 in the form of Fig. 27(b), where $\boldsymbol{E}(z)=\left[E_{k n}^{(z)}\right], 0 \leq k, n, \leq M-1$, and $\boldsymbol{R}(z)=\left[R_{n k}^{(z)}\right], 0 \leq$ $k, n, \leq M-1$. Now we can employ the noble identities (Fig. 9) to move the decimator and interpolator, resulting in Fig. 27(c), where $\boldsymbol{P}(z)=\boldsymbol{R}(z) \boldsymbol{E}(z)$.

It is now clear that if the filters $H_{k}(z)$ and $F_{k}(z)$ are such that

$$
\boldsymbol{R}(z)=\boldsymbol{E}^{-1}(z)
$$

then the system of Fig. 27(c) reduces to that of Fig. 27(a), which is a PR system! It is in fact shown in [31] that a necessary and sufficient condition for the PR property is that $P(z)$ should have one of the following forms:

$$
P(z)=d\left[\begin{array}{ll}
z^{-\kappa} & 0 \\
0 & z^{-\kappa}
\end{array}\right], \quad \text { or } d\left[\begin{array}{ll}
0 & z^{-\kappa} \\
z^{-\kappa-1} & 0
\end{array}\right] .
$$

Returning now to the earlier example, where the filters were related as in (34) and (35), we have

$$
\boldsymbol{E}(z)=\left[\begin{array}{rr}
E_{0}(z) & E_{1}(z) \\
E_{0}(z) & -E_{1}(z)
\end{array}\right], \quad \boldsymbol{R}(z)=\left[\begin{array}{cc}
E_{1}(z) & E_{1}(z) \\
E_{0}(z) & -E_{0}(z)
\end{array}\right]
$$

where $E_{0}(z)$ and $E_{1}(z)$ are polyphase components of $H_{0}(z)$. We then have

$$
\boldsymbol{R}(z) \boldsymbol{E}(z)=2 E_{0}(z) E_{1}(z) \boldsymbol{l}
$$


As explained earlier, it is not possible to obtain a PR system based on this, because $H_{0}(z)$ would then become constrained as in (37).

To obtain an FIR PR system with good filters $H_{0}(z)$ and $H_{1}(z)$, we have to give up the constraint (34). Smith and Barnwell [54] have shown how this can be done, based on spectral factorization of half-band filters. We shall review this same approach from a different viewpoint which enables us to extend the perfect reconstruction property for arbitrary $M$ and; in addition, gives efficient polyphase lattice structures for robust implementation. Consider the task of satisfying the general condition (45) for perfect reconstruction. Thus let us force the product $\boldsymbol{P}(z)$ to be $d z^{-K} I$ by requiring $\boldsymbol{R}(z)=d z^{-K} \boldsymbol{E}^{-1}(z)$. This means that $\boldsymbol{R}(z)$ and hence $F_{k}(z)$ are, in general, IIR filters. The synthesis filters will be stable provided the determinant of $\boldsymbol{E}(z)$ is a minimum phase polynomial.

To obtain a PR system with only FIR filters, we must work harder. The strategy woud be to constrain det $E(z)$ to be a delay. A subclass of FIR matrices for which the determinant is a delay is the family of lossless matrices [31], [50]. To design FIR PR QMF banks systematically, we now turn attention to this family. It should be remembered, however, that losslessness of $E(z)$ is not a necessary condition for perfect reconstruction [30], [31], [55].

\section{Lossless Systems ${ }^{2}$}

Historically, lossless systems are well known in classical electrical network theory [56]-[58]. An electrical network composed of lossless elements (such as inductors and capacitors but no resistors) is said to be lossless. The impedance matrix $Z(s)$ of a multiterminal lossless electrical network satisfies $\operatorname{Re}[Z(j \Omega)]=0$. The scattering matrix of a multiterminal network is defined as $T(s)=[Z(s)-I][Z(s)+I]^{-1}$ For a lossless network, the property of $Z(j \Omega)$ translates into the unitariness of $T(j \Omega)$. An excellent (and perhaps the only one of its kind) reference on lossless systems is the text by Belevitch [58].

Interestingly enough, the concepts of losslessness and passivity have played a crucial role in single-rate digital signal processing (primarily in robust digital filter design) [42] [43], [59]-[61]. The importance of lossless systems in multirate digital filter banks has been exploited in [31]. Our presentation in this tutorial is based on [31], [50], and [62]-[65], which deal with discrete-time lossless systems directly in the $z$ domain, for application in QMF bank design.

Discrete-Time Lossless Systems: A $p \times r$ transfer matrix $\boldsymbol{H}(z)$ describes an $r$-input $p$-output system with input vector $u(n)$ and output vector $y(n)$. The $z$-transforms of $u(n)$ and $y(n)$ are related as $\boldsymbol{Y}(z)=\boldsymbol{H}(z) \boldsymbol{U}(z)$. The energy of the sequences are defined as $E_{u}=\Sigma_{n=-\infty}^{\infty} \boldsymbol{u}^{\dagger}(n) \boldsymbol{u}(n)$ and $E_{y}=\Sigma_{n=-\infty}^{\infty} \boldsymbol{y}^{\dagger}(n)$ $\boldsymbol{y}(n)$, respectively. The system $\boldsymbol{H}(z)$ is said to be lossless if the relation $E_{y}=c E_{u}$ holds for every possible input sequence, where $c>0$ is a constant independent of $u(n)$. If we take $c=1$, then the output of the lossless system has the same energy as the input, justifying the name.

More formally, a transfer matrix $\boldsymbol{H}(\mathrm{z})$ is said to be lossless if it is stable (i.e., all entries have poles inside the unit circle [7]) and satisfies

$$
\tilde{\boldsymbol{H}}(\mathrm{z}) \boldsymbol{H}(\mathrm{z})=\mathrm{cl} \quad \text { for all } \mathrm{z},
$$

${ }^{2}$ At this point, the reader may find it beneficial to review the Nomenclature section, found at the end of the paper. for some $c \neq 0$. The property of $(48)$ implies that $\boldsymbol{H}(z)$ is unitary on the unit circle (see section I), i.e., $\boldsymbol{H}^{\dagger}\left(\mathrm{e}^{j \omega}\right) \boldsymbol{H}\left(\mathrm{e}^{j \omega}\right)=$ $c l$ for all $\omega$.

A constant unitary matrix is a trivial example of a lossless system. A scalar stable all-pass function $H(z)$, which by definition satisfies $\left|H\left(e^{j \omega}\right)\right|=$ constant, is another example. As a third example, if $H_{k}(z), 0 \leq k \leq M-1$ is a power complementary set, then the $M \times 1$ vector $\left[H_{0}(z) H_{1}(z) \cdots\right.$ $\left.H_{M-1}(z)\right]^{T}$ is a lossless system. A lossless example with $p=$ $r$ is offered by the $2 \times 2$ orthogonal matrix $\left[\begin{array}{cc}1 & -\alpha \\ 1 & 1\end{array}\right]$. When $p$ $=r$, an FIR lossless systems $\boldsymbol{H}(\mathrm{z})$ satisfies the property det $\boldsymbol{H}(z)=d z^{-K}$, where $\boldsymbol{d}$ is a constant and $K$ is an integer [62], [64]. Moreover, the inverse is given by $\boldsymbol{H}^{-1}(z)=\tilde{\boldsymbol{H}}(\mathrm{z}) / c$, as seen from (48). In other words, the inverse is not only guaranteed to exist, but also can be found from $\boldsymbol{H}(\mathrm{z})$ simply by writing down $\tilde{\boldsymbol{H}}(\mathrm{z})$. For example, let

$$
H(z)=\left[\begin{array}{ll}
1+z^{-1} & 1-z^{-1} \\
1-z^{-1} & 1+z^{-1}
\end{array}\right] .
$$

We can verify that this is lossless (because (48) is satisfied with $c=4$ ). The inverse is given by

$$
H^{-1}(z)=\frac{1}{4} \tilde{H}(z)=\frac{1}{4}\left[\begin{array}{ll}
1+z & 1-z \\
1-z & 1+z
\end{array}\right] .
$$

\section{Use of Lossless Systems in QMF Design}

Returning now to the QMF bank, suppose we restrict $E(z)$ to be FIR and lossless. We can now choose $R(z)$ as

$$
\boldsymbol{R}(z)=z^{-\kappa} \tilde{E}(z)=c z^{-\kappa} E^{-1}(z)
$$

so that perfect reconstruction is guaranteed. The delay $z^{-k}$ ensures causality of the synthesis bank. For example, if $E(z)$ is as in (49), then $R(z)$ should be

$$
\boldsymbol{R}(z)=\left[\begin{array}{rr}
1+z^{-1} & -1+z^{-1} \\
-1+z^{-1} & 1+z^{-1}
\end{array}\right]
$$

With $E(z)$ as in (49) and $R(z)$ as in (52), we have

$$
\begin{aligned}
H_{0}(z)=1+z^{-1}+z^{-2}-z^{-3}, \\
H_{1}(z)=1+z^{-1}-z^{-2}+z^{-3}
\end{aligned}
$$

and

$$
\begin{gathered}
F_{0}(z)=\left[-1+z^{-1}+z^{-2}+z^{-3}\right], \\
F_{1}(z)=\left[1-z^{-1}+z^{-2}+z^{-3}\right] .
\end{gathered}
$$

Thus the simple choice of filters, as in (53) and (54), results in an FIR PR QMF bank.

The product of paraunitary systems is paraunitary. Therefore the product of lossless systems is lossless, so that we can generate more complicated examples of arbitrary degree just by multiplying together simple examples of $\boldsymbol{E}(z)$. However, to obtain a systematic procedure for the design of QMF banks based on lossless polyphase matrix $\boldsymbol{E}(z)$, we have to find a technique for characterizing the entire family of $2 \times 2$ FIR lossless systems. By performing a systematic gradient search within this family, it is then possible to optimize the filter responses $H_{0}(z)$ and $H_{1}(z)$ so that they have good stopband attenuations. In preparation for this we shall now summarize a number of important results pertaining to the QMF bank based on lossless $E(z)$. See [65] for most proofs. 
1) The four entries of a $2 \times 2$ FIR lossless system are related to each other because of the constraint $\tilde{\boldsymbol{E}}(z)$ $E(z)=c l$. It can be shown that the most general $2 \times$ 2 causal FIR lossless system has the form

$$
\boldsymbol{E}(z)=\left[\begin{array}{cc}
E_{00}(z) & \alpha z^{-K} \tilde{E}_{10}(z) \\
E_{10}(z) & -\alpha z^{-K} \tilde{E}_{00}(z)
\end{array}\right]
$$

where $\alpha$ is a constant with $|\alpha|=1, K \geq 0$ is an integer large enough to make the entries causal, and $\left\{E_{00}(z)\right.$, $\left.E_{10}(z)\right\}$ forms a power complementary pair. In what follows we assume $\alpha=1$, as this does not reduce the freedom for filter design.

2) As a consequence of this, if we use $\boldsymbol{E}(z)$ to construct analysis filters as in (42), then $H_{1}(z)$ is related to $H_{0}(z)$ by

$$
H_{1}(z)=-z^{-(2 K+1)} \tilde{H}_{0}(-z) .
$$

Here $2 K+1$ is the order $N-1$ of $H_{0}(z)$ (so that $H_{1}(z)$ in (56) is causal). With this, the coefficients of $H_{1}(z)$ are given by

$$
h_{1}(n)=(-1)^{n} h_{0}^{*}(N-1-n) .
$$

3) With the matrix $R(z)$ chosen as $z^{-\kappa} \tilde{E}(z)$ the synthesis filters determined by (43) are given as

$$
F_{0}(z)=z^{-(N-1)} \tilde{H}_{0}(z), \quad F_{1}(z)=z^{-(N-1)} \tilde{H}_{1}(z)
$$

so that

$$
f_{0}(n)=h_{0}^{*}(N-1-n), \quad f_{1}(n)=h_{1}^{*}(N-1-n) .
$$

Clearly this choice also implies $\left|F_{0}\left(\mathrm{e}^{j \omega}\right)\right|=\left|H_{0}\left(\mathrm{e}^{j \omega}\right)\right|$ and $\left|F_{1}\left(\mathrm{e}^{j \omega}\right)\right|=\left|H_{1}\left(\mathrm{e}^{j \omega}\right)\right|$.

4) With $\boldsymbol{E}(z)$ constrained to be lossless, the pair $\left[H_{0}(z)\right.$, $\left.H_{1}(z)\right]$ is power complementary, and so is the pair $\left[F_{0}(z)\right.$, $\left.F_{1}(z)\right]$.

5) The quantity $\tilde{H}_{0}(z) H_{0}(z)$ is a zero-phase half-band filter (section $\mathrm{V}$-A).

Summarizing, an FIR PR QMF bank with $\boldsymbol{E}(z)$ chosen to be lossless has the following properties: a) $\left[H_{0}(z), H_{1}(z)\right]$ is a power complementary pair; b) $H_{1}(z)$ is related to $H_{0}(z)$ by (56), i.e., by (57); and c) the synthesis filters are given by (58), i.e., by (59). Conversely, it turns out [65] that if an FIR QMF bank satisfies these three properties, it is a perfect reconstruction system.

It can finally be shown that if $H_{0}(z)$ is any FIR transfer function such that its polyphase components $E_{00}(z)$ and $E_{01}(z)$ are power-complementary (or, equivalently, if $\tilde{H}_{0}(z) H_{0}(z)$ is a zero-phase half-band filter; see section $\mathrm{V}-\mathrm{C}$ ), then the following things are true: a) the matrix $E(z)$ defined as (55) is lossless; b) if $H_{1}(z), F_{0}(z)$, and $F_{1}(z)$ are chosen as in (56) and (58), we have a FIR PR QMF system.

The Design Procedure: in the aforementioned FIR PR QMF system, once we know $H_{0}(z)$, the remaining transfer functions $H_{1}(z), F_{0}(z)$, and $F_{1}(z)$ are completely determined. We are now left with one final question: how to design the coefficients $h_{0}(n)$ of $H_{0}(z)$ ? We shall restrict the design to the case of real $h_{0}(n)$. It is shown in [62] that every $2 \times 2$ realcoefficient FIR lossless system $\boldsymbol{E}(z)$ can be expressed in the form

$$
\boldsymbol{E}(\mathrm{z})=d \boldsymbol{R}_{\mathrm{K}} \mathbf{\Lambda}(\mathrm{z}) \boldsymbol{R}_{\mathrm{K}-1} \mathbf{\Lambda}(\mathrm{z}) \cdots \boldsymbol{R}_{1} \mathbf{\Lambda}(\mathrm{z}) \boldsymbol{R}_{0}
$$

where $\boldsymbol{R}_{n}$ are $2 \times 2$ real orthogonal matrices of the form

$$
\boldsymbol{R}_{n}=\left[\begin{array}{cc}
1 & -\alpha_{n} \\
\alpha_{n} & 1
\end{array}\right],
$$

$\boldsymbol{\Lambda}(z)$ is a diagonal matrix of the form

$$
\mathbf{\Lambda}(z)=\left[\begin{array}{ll}
1 & 0 \\
0 & z^{-1}
\end{array}\right],
$$

and $d$ is a real scalar constant of no serious consequence. Here $K$ is the degree of $\boldsymbol{E}(\mathrm{z})$. Note that the building blocks (61) and (62) in the product (60) are themselves lossless. With $E(z)$ used as the polyphase matrix for the analysis filters and with the synthesis-bank polyphase matrix chosen as (51), any real-coefficient FIR PR QMF bank with lossless $\boldsymbol{E}(z)$ can be drawn as in Fig. 28. This is called the lattice structure,
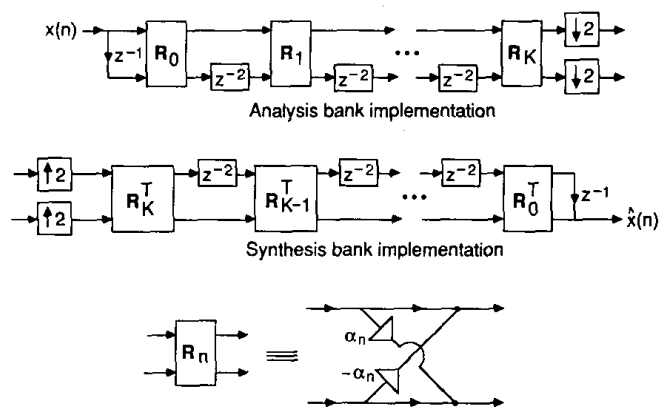

Fig. 28. Lattice structure implementation of two-channel real-coefficient FIR perfect reconstruction QMF bank with lossless $\boldsymbol{E}(\mathrm{z})$.

because of the criss-cross nature of the building blocks. The parameters $\alpha_{n}$ can be optimized on a computer to ensure that the stopband energy of $H_{0}(z)$ is minimized; the passband of $H_{0}(z)$ comes out to be automatically good because of the half-band property of $\tilde{H}_{0}(z) H_{0}(z)$. Several design examples can be found in [65], along with tables of optimal filter coefficients with filters designed in this manner. The use of filters related by (56) and (58) (with $H(z) \triangleq \tilde{H}_{0}(z) H_{0}(z)$ constrained to be a half-band filter) in the design of perfect reconstruction QMF banks was first recognized in [54] and [66]. The first approach to design such filters was based on the design of the half-band filter $H(z)$ followed by spectral factorization. Such factorization is often numerically inaccurate because $H(z)$ has large attenuation and typically has several zeros on the unit circle. An inaccurate spectral factor results in a QMF bank with imperfect reconstruction property. Later on the lattice structure of Fig. 28 was introduced so that a direct optimization of $H_{0}(z)$ could be done with no need for factorization; the lattice guarantees perfect reconstruction despite coefficient quantization.

A noteworthy feature of the lattice is that regardless of the values of $\alpha_{n}$ and $d$, the following properties hold: a) $\tilde{H}_{0}(z) H_{0}(z)$ is a zero-phase half-band filter; b) $H_{1}(z)$ is related to $H_{0}(z)$ by $\left.(56) ; c\right)\left[H_{0}(z), H_{1}(z)\right]$ is power complementary; and d) $\hat{x}(n)=c x(n-2 K-1)$, so that perfect reconstruction is guaranteed regardless of quantization of the coefficients $\alpha_{n}$.

Linear-Phase FIR Perfect Reconstruction OMF Banks: In some perfect reconstruction sytems, it is desirable to have 
analysis and synthesis filters having linear phase. At first this might appear to be a redundant requirement because, the complete analysis/synthesis system has the PR property anyway (which means that any phase distortion caused by analysis filters is somehow compensated for by the synthesis filters). However, in practice, the term "PR system" implies that only the linear errors (ALD, AMD, and PHD) are eliminated but not the nonlinear coding error (which results from the coding of the subband signals after decimation). For low bit-rate coding, the coding errors are typically correlated to the signals, and the alias cancellation is not perfect in the presence of such errors. Experience has indicated (particularly in image-processing applications) that the reconstruction error caused by this coding error is less harmful if the analysis filters used in the QMF bank have linear phase.

The perfect reconstruction system described in the preceding is such that the analysis filters $H_{k}(z)$ do not have linear phase. This is because a linear-phase power complementary pair $\left[H_{0}(z), H_{1}(z)\right]$ necessarily has trivial forms (such as (37)). If we give up the power complementary requirement of the analysis filters, we can obtain perfect reconstruction FIR QMF banks with linear-phase analysis filters (and synthesis filters of same lengths). Two such families are reported in [67], where the losslessness of $E(z)$ (and hence the power-complementarity of the analysis filters) is given up for this purpose. The resulting analysis-bank structure is somewhat similar to Fig. 28. The main difference is that the minus sign in front of $\alpha_{k}$ in each building block is absent for $0 \leq n \leq K-1$ (so that $R_{n}$ is not orthogonal any more). The last section $\boldsymbol{R}_{K}$ is, however, exactly as in Fig. 28, with $\alpha_{K}=1$. Further detailed derivations, design examples, and the exact details about the corresponding synthesis bank can be found in [67].

\section{E. Computational Complexity and Price Paid for Perfect Reconstruction}

So far in this paper we have seen three methods for the design of two-channel QMF banks. In one method, the relations (34) and (35) are satisfied, and $H_{0}(z)$ is a linear-phase FIR filter; ALD and PHD are completely eliminated; and AMD is minimized using Johnston's procedure [4], [48]. In another method, relations (34) and (35) are still satisfied, but $H_{0}(z)$ is an IIR filter of the form (39a), where $A_{0}(z)$ and $A_{1}(z)$ are allpass filters of the form (39b); ALD and AMD are completely eliminated; and PHD reduced by all-pass equalization.

We then described a method where the analysis and synthesis filters are FIR, satisfying a different relation (viz., (56) and (58)), and are such that $\tilde{H}_{0}(z) H_{0}(z)$ has half-band property. In this method, ALD, AMD, and PHD are completely eliminated, but the analysis filters do not have linear phase. Finally, we mentioned a method which gives rise to FIR perfect reconstruction systems in which the analysis filters have linear phase (while at the same time offering arbitrarily good attenuation characteristics).

Several features of the preceding four methods are summarized in Table 1, where a number is assigned to each method for identification purposes. Notice in particular the MPU count (derived in the following) for the entire QMF bank, which is equal to the length of the filter $H_{0}(z)$ for methods 1 and 2, and half this length for method 3 . The group delay of the complete QMF system is also tabulated, and is equal to the order of $H_{0}(z)$ for the first three methods.

Table 1 Comparison of Four Types of Two-Channel QMF Banks.

\begin{tabular}{|c|c|c|c|c|}
\hline & $\begin{array}{c}\text { Method } 1 \\
\text { (Johnston's Table) } \\
{[4],[48]}\end{array}$ & $\begin{array}{c}\text { Method 2 } \\
\text { (Smith-Barnwell [54], } \\
\text { Vaidyanathan/Hoang } \\
\text { [65]) } \\
\text { Perfect-Reconstruction } \\
\text { System }\end{array}$ & $\begin{array}{c}\text { Method } 3 \\
\text { (Vaidyanathan/Nguyen [67]) } \\
\text { Perfect-Reconstruction } \\
\text { System }\end{array}$ & $\begin{array}{c}\text { Method } 4 \\
\text { IIR Based } \\
{[12],[43]}\end{array}$ \\
\hline $\begin{array}{l}\text { Relation between } \\
\text { filters }\end{array}$ & $\begin{array}{l}H_{1}(z)=H_{0}(-z) \\
F_{0}(z)=H_{0}(z) \\
F_{1}(z)=-H_{0}(-z)\end{array}$ & $\begin{array}{l}H_{1}(z)=-z^{-1} \tilde{H}_{0}(-z) \\
F_{0}(z)=z^{-L} \bar{H}_{0}(z) \\
F_{1}(z)=z^{-L} \bar{H}_{1}(z) \\
L=\text { order of } H_{0}(z)\end{array}$ & $\begin{array}{l}\text { not explicit; implicitly } \\
\text { det } \boldsymbol{E}(z)=\text { delay, and } \\
R(z)=c z^{-\alpha} E^{-1}(z)\end{array}$ & same as method 1 \\
\hline $\begin{array}{l}\text { Phase response of } \\
\qquad H_{0}(z)\end{array}$ & linear & nonlinear & linear & $\begin{array}{l}\text { nonlinear since } H_{0}(z) \\
\text { IIR }\end{array}$ \\
\hline $\begin{array}{l}\text { Other crucial features } \\
\text { of } H_{0}(z)\end{array}$ & & $\begin{array}{l}\tilde{H}_{0}(z) H_{0}(z) \text { is a (zero- } \\
\text { phase FIR) half-band } \\
\text { filter }\end{array}$ & & $\begin{array}{l}2 H_{0}(z)=a_{0}\left(z^{2}\right) \\
\quad+z^{-1} a_{1}\left(z^{2}\right), a_{0}(z) \\
\quad a_{1}(z) \text { IIR allpass }\end{array}$ \\
\hline $\begin{array}{l}\text { Distortions in QMF } \\
\text { bank }\end{array}$ & $\begin{array}{l}\text { ALD canceled } \\
\text { AMD minimized } \\
\text { PHD eliminated }\end{array}$ & $\begin{array}{l}\text { ALD canceled } \\
\text { AMD eliminated } \\
\text { PHD eliminated }\end{array}$ & $\begin{array}{l}\text { ALD canceled } \\
\text { AMD eliminated } \\
\text { PHD eliminated }\end{array}$ & $\begin{array}{l}\text { ALD canceled } \\
\text { AMD eliminated } \\
\text { PHD equalized }\end{array}$ \\
\hline $\begin{array}{l}\text { Notation for filter } \\
\text { length or order as } \\
\text { relevant }\end{array}$ & $\begin{array}{c}N_{1}=\text { length of } \\
H_{0}(z)\end{array}$ & $N_{2}=$ length of $H_{0}(z)$ & $N_{3}=$ length of $H_{0}(z)$ & $N_{4}=$ order of $H_{0}(z)$ \\
\hline $\begin{array}{l}\text { Number of MPU to } \\
\text { implement entire } \\
\text { analysis/synthesis } \\
\text { system }\end{array}$ & $\begin{array}{c}N_{1} \text { (direct-form, } \\
\text { polyphase) }\end{array}$ & $\mathrm{N}_{2}$ (lattice, polyphase) & $\frac{N_{3}}{2}$ (lattice, polyphase) & $\begin{array}{l}\frac{\mathrm{N}_{4}-1}{2}+\text { equalizer } \\
\text { overhead (all-pass. } \\
\text { polyphase) }\end{array}$ \\
\hline $\begin{array}{l}\text { Group delay of entire } \\
\text { analysis/synthesis } \\
\text { system }\end{array}$ & $N_{1}-1$ & $N_{2}-1$ & $N_{3}-1$ & complicated \\
\hline
\end{tabular}


Counting the Number of MPU: For Method 1 the MPU count is obvious from earlier discussions. For Method 2, note that the lattice structure of Fig. 28 enables us to obtain a polyphase implementation requiring half as many MPUs as a direct-form implementation of the analysis bank. This is because each section of the form (61) requires two multipliers, which operate at half the input rate (because the decimators can be moved all the way to the left in Fig. 28), and the number of such sections in the analysis bank is equal to $N / 2$, where $N$ is the length of $H_{0}(z)$. Consequently, the entire analysis/synthesis system requires only NMPUs. A direct-form implementation would have required $2 \mathrm{~N}$ $\mathrm{MPU}$, and the modified direct form [68] would require $1.5 \mathrm{~N}$ MPUs. Next, for Method 3 the same lattice structure can be used with the modification mentioned in section VI-D. Since this modification calls for deletion of the minus sign in front of $\alpha_{k}$ in Fig. 28, each lattice section requires only one (rather than two) multiplier [69], so the MPU count is reduced by a factor of two. For method 4, the MPU count is obtained from the fact that any all-pass function of order $L$ can be implemented with only $L$ multipliers [53].

To obtain a more complete feeling for the relative tradeoffs and complexities of the various methods, we compare them based on design examples of identical specifications (as far as practicable). Table 2 compares the three FIR QMF banks. In each method, $H_{0}(z)$ is required to have a stopband attenuation $A_{s} \approx 38 \mathrm{~dB}$, and stopband edge $\omega_{s} \approx 0.586 \pi$. Only method 1 has amplitude distortion, and this is proportional to the second integral in (38) (see [48] for quantitative definition). Assume that this distortion is required to be $0.025 \mathrm{~dB}$ or less. With these requirements, methods 1 and 2 require approximately the same filter length, and hence the same MPU count. On the other hand, method 3 (where the analysis filters have linear phase and the QMF bank has perfect reconstruction) requires a higher length of 62 . However, because of the existence of a neat lattice representation for method 3 , the number of MPUs required for the entire QMF bank is nearly same as for methods 1 and 2 !

If this is so, what is the price paid for perfect reconstruction? Compared to method 1 , method 2 has nonlinear-analysis filters, which can be considered the price paid. How- ever, this is overcome in method 3. The only price paid in method 3 is that the overall group delay of the QMF system is two times longer. Assuming an input sampling rate of 8 $\mathrm{kHz}$ for $x(n)$ in Fig. 13, this group delay is approximately 4 $\mathrm{ms}$ for methods 1 and 2 , and $8 \mathrm{~ms}$ for method 3 . Even $8 \mathrm{~ms}$ is unlikely to be objectionable in most speech communication systems.

Next, Table 3 shows a comparison of the FIR perfect reconstruction system (method 2) with the IIR QMF bank

Table 3 Comparison of Methods 2 and 4, When Both are Required to have $A_{s} \simeq 40 \mathrm{~dB}$, and $\omega_{\mathrm{s}}=0.62 \pi$

\begin{tabular}{lcc}
\hline & $\begin{array}{c}\text { Method } 2 \\
\text { (Perfect- } \\
\text { Reconstruction } \\
\text { FIR System) }\end{array}$ & $\begin{array}{c}\text { Method } 4 \\
\text { (IIR System with } \\
\text { Nonzero PHD) }\end{array}$ \\
\hline $\begin{array}{l}\text { Filter length or order } \\
\text { as appropriate }\end{array}$ & $\begin{array}{c}N_{2}=\text { filter length } \\
\text { for } H_{0}(z)=22\end{array}$ & $\begin{array}{c}N_{4}=\text { order of } \\
H_{0}(z)=5\end{array}$ \\
$\begin{array}{l}\text { Number of MPU for } \\
\text { entire analysis/ } \\
\text { synthesis system }\end{array}$ & 22 & $\begin{array}{c}11, \text { with an allpass } \\
\text { equalizer } E(z) \text { of } \\
\text { order } 9\end{array}$ \\
$\begin{array}{l}\text { Group delay } \\
\text { distortion }\end{array}$ & none & $\begin{array}{c}2.5 \text { samples with } \\
\text { allpass equalizer } \\
E(z) \text { of order } 9\end{array}$ \\
Other distortions & none & none
\end{tabular}

(method 4) with $H_{0}(z)$ constrained to have $A_{s} \approx 40 \mathrm{~dB}$ and $\omega_{5}=0.62 \pi$ in both cases. With a ninth-order all-pass equalizer, the group delay distortion of method 4 is about 2.5 samples and the MPU count is only half as much as the FIR perfect reconstruction system. Note, however, that for systems with sharper $H_{0}(z)$, the equalizer tends to have disproportionately higher order, so that the IIR system might end up having a higher MPU count!

VII. Unification of Alias-Free QMF Banks, Block Digital Filters and LPTV Systems

There are three topics in digital signal processing, developed quite independently of each other, that are closely related by a common mathematical structure [70]. These topics are alias-free QMF banks, block digital filtering, and

Table 2 Comparison of Design Examples for Three FIR Two-Channel QMF Banks. In All Three Methods, $H_{0}(z)$ has $A_{s} \simeq 38 \mathrm{~dB}$ and $\omega_{\mathrm{s}} \simeq 0.586 \pi$

\begin{tabular}{|c|c|c|c|}
\hline & $\begin{array}{c}\text { Method 1 } \\
\text { (Johnston's Tables) } \\
\text { i.e., Imperfect Reconstruction } \\
\text { with Linear Phase } \\
\text { Analysis and Synthesis Filters }\end{array}$ & $\begin{array}{c}\text { Method } 2 \\
\text { (Vaidyanathan-Hoang) } \\
\text { i.e., Perfect-Reconstruction } \\
\text { with Nonlinear Phase } \\
\text { Analysis and Synthesis } \\
\text { Filters }\end{array}$ & $\begin{array}{c}\text { Method 3 } \\
\text { (Nguyen-Vaidyanathan) } \\
\text { i.e., Perfect-Reconstruction } \\
\text { with Linear Phase } \\
\text { Analysis and Synthesis } \\
\text { Filters }\end{array}$ \\
\hline $\begin{array}{l}\text { Required filter-length } \\
\text { for } H_{0}(z)\end{array}$ & $\begin{array}{l}N_{1}=32 \text { for AMD error } E_{r}= \\
0.025 \mathrm{~dB}\end{array}$ & $N_{2}=30$ & $N_{3}=62$ \\
\hline $\begin{array}{l}\text { Group delay of entire } \\
\text { analysis/synthesis } \\
\text { system }\end{array}$ & 31 & 29 & 61 \\
\hline $\begin{array}{l}\text { Number of MPU for } \\
\text { entire analysis/ } \\
\text { synthesis system }\end{array}$ & 32 & 30 & 31 \\
\hline $\begin{array}{l}\text { Price paid by the } \\
\text { method in relation } \\
\text { to remaining two } \\
\text { methods }\end{array}$ & AMD not equal to zero & $\begin{array}{c}H_{0}(z) \text { and } H_{1}(z) \text { do not } \\
\text { have linear phase }\end{array}$ & Group delay is longer \\
\hline
\end{tabular}


linear periodically time-varying (LPTV) systems. In the following parts of this section we shall review this relation.

\section{A. Alias-Free QMF Banks}

Fig. 29(a) shows the $M$ band generalization of the QMF bank. It is said to be a maximally decimated structure because the decimation ratio $M$ is equal to the number of bands. To distinguish this from tree structures (section VIII), the scheme of Fig. 29(a) is called a parallel structure. Once

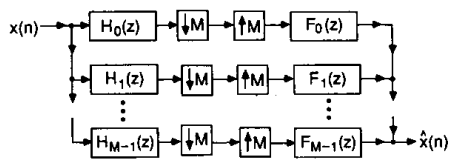

(a)

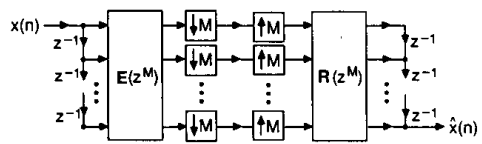

(b)

Fig. 29. (a) $M$-band maximally decimated parallel QMF bank. (b) Redrawing of Fig. 29(a) in terms of polyphase component matrices.

again, this is an LTV system because of the decimators and interpolators. The correct name for the system of Fig. 29(a) would be " $M$-band maximally decimated analysis/synthesis system." It is, however, loosely called the "QMF" bank in analogy with the two-channel system of Fig. 13. For the twochannel case, the response $\left|H_{0}\left(\mathrm{e}^{j \omega}\right)\right|$ is usually an image of $\left|H_{1}\left(e^{j \omega}\right)\right|$ with respect to the quadrature frequency $\pi / 2$, justifying the name "quadrature mirror filter." For the $M$-band case, the name QMF is technically illogical, but has been used for simplicity by several authors.

If there were no decimators and interpolators in Fig. 29(a), it would have been very easy to obtain perfect reconstruction by using any one of the special techniques of section $\mathrm{V}$. In the presence of the decimators and interpolators, we must be more careful, due to aliasing effects. Employing (3a) and ( $3 b)$, we arrive at the following relation, which reflects the aliasing caused by the decimators and the imaging caused by the interpolators:

$$
\hat{X}(z)=\frac{1}{M} \sum_{k=0}^{M-1} \sum_{n=0}^{M-1} F_{k}(z) H_{k}\left(z W^{n}\right) X\left(z W^{n}\right)
$$

This shows that there are $M-1$ alias components $X\left(z W^{n}\right)$, $n>0$ in the reconstructed signal, besides the original $X(z)$. If these components are eliminated by proper choice of $F_{k}(z)$ and $H_{k}(z)$, the QMF bank becomes an LTI system with transfer function $T(z)=\hat{X}(z) / X(z)=\Sigma_{k=0}^{M-1} F_{k}(z) H_{k}(z) / M$. Furthermore, if $T(z)$ is all-pass, AMD is eliminated, whereas if $T(z)$ is linear-phase FIR, PHD is eliminated.

The condition for alias-cancellation can be written in matrix form [12], [71], [72] as

$$
\left[\begin{array}{ccccc}
H_{0}(z) & H_{1}(z) & \cdots & H_{M-1}(z) \\
H_{0}(z W) & H_{1}(z W) & \cdots & H_{M-1}(z W) \\
\vdots & \vdots & \cdots & \vdots \\
H_{0}\left(z W^{M-1}\right) & H_{1}\left(z W^{M-1}\right) & \cdots & H_{M-1}\left(z W^{M-1}\right)
\end{array}\right]\left[\begin{array}{c}
F_{0}(z) \\
F_{1}(z) \\
\vdots \\
F_{M-1}(z)
\end{array}\right]=\left[\begin{array}{c}
M T(z) \\
0 \\
\vdots \\
0
\end{array}\right] .
$$

The $M \times M$ matrix $\boldsymbol{H}(z)$ in (64) is called the alias-component matrix. If it can be inverted, it is indeed possible to find the synthesis filters $F_{k}(z), 0 \leq k \leq M-1$ to cancel aliasing completely. The difficulty with this approach is that even if $H_{k}(z)$ are FIR, the inverse of $\boldsymbol{H}(z)$ could be IIR, so that $F_{k}(z)$ are, in general, neither FIR nor stable. If we multiply all $F_{k}(z)$ by the LCM of the denominators of $F_{k}(z), 0 \leq k \leq M-1$ to get a new set of synthesis filters, aliasing continues to be absent (as seen from (64)), but $T(z)$ changes. In any case, the resulting set of synthesis filters can have very high orders. For these reasons, inversion of $\boldsymbol{H}(z)$ is usually not used as a procedure for the design of alias-free QMF banks.

If we approach this problem in terms of the polyphase matrices $\boldsymbol{E}(z)$ and $\boldsymbol{R}(z)$ as in section VI-B, we have much better luck. In fact, a convenient procedure for design of $M$-band FIR perfect-reconstruction QMF banks based on lossless $\boldsymbol{E}(z)$ has been developed [31], [63], [64], and will be summarized in Section VIII.

Suppose we express $H_{k}(z)$ and $F_{k}(z)$ in terms of Type 1 and Type 2 polyphase components, respectively:

$$
\begin{aligned}
H_{k}(z)=\sum_{n=0}^{M-1} z^{-n} E_{k n}\left(z^{M}\right), \\
F_{k}(z)=\sum_{n=0}^{M-1} z^{-(M-1-n)} R_{n k}\left(z^{M}\right) .
\end{aligned}
$$

Defining the $M \times M$ matrices $\boldsymbol{E}(z)=\left[E_{k n}(z)\right]$ and $\boldsymbol{R}(z)=$ $\left[R_{n k}(z)\right]$, we can then redraw the QMF bank as in Fig. 29(b). By using the noble identities of Fig. 9, we can move the decimators and interpolators to finally obtain Fig. 30, where $\boldsymbol{P}(z)$

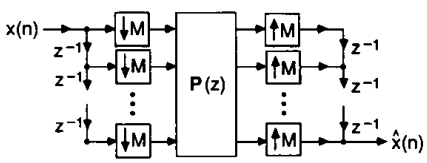

Fig. 30. Equivalent structure of Fig. 29.

$=\boldsymbol{R}(z) \boldsymbol{E}(z)$. In conclusion, every $M$-band maximally decimated QMF bank can be redrawn as in Fig. 30. The properties of the QMF bank, such as aliasing, and other distortions depend completely on the behavior of $\boldsymbol{P}(z)$. We now ask our first question in this section (and discuss the answer in Section VIII-D).

Question 1: What is a set of necessary and sufficient conditions in terms of $\boldsymbol{P}(z)$ so that aliasing is completely canceled?

\section{B. Block Digital Filters}

Consider a single-input single-output (or "scalar") LTI system with transfer function $S(z)$ (Fig. 31(a)). From the input sequence $x(n)$ let us create a vector sequence $x(n)$ as follows:

$$
\boldsymbol{x}(n)=\left[\begin{array}{llll}
x_{M-1}(n) & x_{M-2}(n) & \cdots & x_{0}(n)
\end{array}\right]^{T}
$$




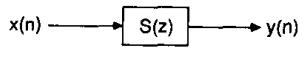

(a)

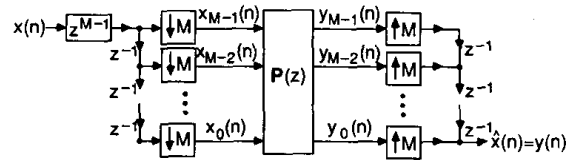

(b)

Fig. 31. (a) Scalar transfer function $S(z)$. (b) Its blocked version with block length $M$.

where $x_{k}(n)=x(n M+k), 0 \leq k \leq M-1$. Then $x(n)$ is called the blocked version of the scalar signal $x(n)$. Similarly, the blocked version $y(n)$ of $y(n)$ is

$$
y(n)=\left[\begin{array}{llll}
y_{M-1}(n) & y_{M-2}(n) & \cdots & y_{0}(n)
\end{array}\right]^{T}
$$

where $y_{k}(n)=y(n M+k), 0 \leq k \leq M-1$. The integer $M$ is called the block length. It can be shown [73]-[75] that the two vector-sequences $y(n)$ and $x(n)$ are related by a transfer matrix, i.e.,

$$
Y(z)=P(z) X(z)
$$

The $M \times M$ matrix $\boldsymbol{P}(z)$ is called the blocked version of $S(z)$. Implementing the filter $S(z)$ by direct implementation of $\boldsymbol{P}(z)$ is called block digital filtering. The advantage of such implementation is that we increase the available parallelism by a factor of $M$. Block filtering therefore offers higher implementation speed due to increased parallelism.

We can see that the blocking operation (68) is very closely related to polyphase decomposition. Indeed, the components of (66) are merely $M$-fold decimated versions of $x(n$ $+k), 0 \leq k \leq M-1$. It can be seen that the implementation of $S(z)$ using the blocking approach can be given a flowgraph representation as in Fig. 31(b). The close relation between the blocking scheme and QMF banks can be seen from this representation. Here is the second question we raise in this section (we shall discuss the answer in the following text).

Question 2: Suppose we are given an arbitrary $M \times M$ transfer matrix $\boldsymbol{P}(z)$. What is a set of necessary and sufficient conditions on $\boldsymbol{P ( z )}$ such that it is the blocked version of some scalar filter $S(z)$ ?

\section{Linear Periodically Time-Varying (LPTV) Systems}

Consider a linear system whose coefficients vary periodically with time, with period $M$. For $0 \leq k \leq M-1$, let $A_{k}(z)$ represent the transfer function of the system if its coefficients were frozen to be their values at time $-k$. For each $k$, let us represent $A_{k}(z)$ in terms of its $M$ polyphase components: $A_{k}(z)=\Sigma_{n=0}^{M-1} z^{-n} G_{k n}\left(z^{M}\right)$. Defining $G(z)=\left[G_{k n}(z)\right]$, we can represent the LPTV system as in Fig. 32(a). By defining the components

$$
P_{k n}(z)= \begin{cases}z^{-1} G_{k, M-k+n}(z) & 0 \leq n \leq k-1 \\ G_{k, n-k}(z) & k \leq n \leq M-1\end{cases}
$$

we can redraw Fig. 32(a) as in Fig. 32(b). The relation of (69) between the $k$ th rows of $\boldsymbol{P}(z)$ and $\boldsymbol{G}(z)$ is quite interesting: thus, with $M=3$, we see that $\boldsymbol{P}(z)$ is given by

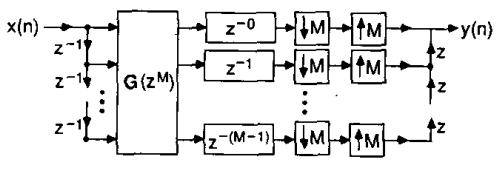

(a)

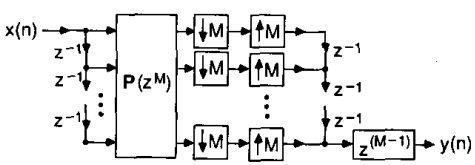

(b)

Fig. 32. Representation of arbitrary linear periodically time varying system using polyphase framework. (a) The LPTV system. (b) Redrawing based on components defined in equation (69).

$$
\boldsymbol{P}(z)=\left[\begin{array}{lll}
G_{00}(z) & G_{01}(z) & G_{02}(z) \\
z^{-1} G_{12}(z) & G_{10}(z) & G_{11}(z) \\
z^{-1} G_{21}(z) & z^{-1} G_{22}(z) & G_{20}(z)
\end{array}\right]
$$

In other words, the $k$ th row of $\boldsymbol{P}(z)$ is a $k$-times circularly shifted version of the $k$ th row of $\boldsymbol{G}(z)$ with a delay $z^{-1}$ attached to the elements that spill over. Here is the third question we raise.

Question 3: Let Fig. 32(b) represent an arbitrary LPTV system. What is a set of necessary and sufficient conditions on $\boldsymbol{P}(z)$ so that the system is actually time-invariant?

\section{Pseudo-circulant Matrices}

The three questions raised in the preceding have the same answer! The answer is that, $\boldsymbol{P}(z)$ should have an algebraic form called the pseudo-circulant form. Thus, if (and only if) $\boldsymbol{P}(z)$ is pseudo-circulant, the following are true: a) the QMF bank is alias-free; b) $\boldsymbol{P}(z)$ is the blocked version of a scalar filter; and c) the LPTV system of Fig. 32(b) represents an LTI system. This result is proved in [70]. In this section we shall merely state the meaning of "pseudo-circulant." Taking $M$ $=3$, for convenience, a $3 \times 3$ pseudo-circulant has the form

$$
P(z)=\left[\begin{array}{lll}
P_{0}(z) & P_{1}(z) & P_{2}(z) \\
z^{-1} P_{2}(z) & P_{0}(z) & P_{1}(z) \\
z^{-1} P_{1}(z) & z^{-1} P_{2}(z) & P_{0}(z)
\end{array}\right]
$$

Thus $\boldsymbol{P}(z)$ is a circulant matrix [76] with the exception that the entries below the main diagonal are multiplied with $z^{-1}$. Pseudo-circulants have also been observed explicitly or implicitly in the context of block processing by other authors [75], [77]-[80].

Assuming that $\boldsymbol{P}(z)$ is pseudo-circulant, what is the scalar filter $S(z)$ of which $P(z)$ is the blocked version? The answer is

$$
S(z)=\sum_{k=0}^{M-1} z^{-k} P_{k}\left(z^{M}\right)
$$

In other words, the 0 th row of $\boldsymbol{P}(z)$ contains the type- 1 polyphase components of $S(z)$. Next, given a QMF bank with pseudo-circulant $\boldsymbol{P}(z)$ (so that aliasing is canceled), the distortion function $T(z)$ of $(64)$ is given by

$$
T(z)=z^{-(M-1)} S(z) \text {. }
$$


It can also be shown that $\boldsymbol{P}(z)$ is lossless if and only if $S(z)$ is stable all-pass. Thus an alias-free QMF bank is free from AMD if and only if the pseudo-circulant is lossless, and the blocked version of a scalar filter is lossless if and only if the scalar filter is stable all-pass!

\section{M-Band Perfect Reconstruction Systems}

Perfect reconstruction in $M$-band FIR QMF banks has been shown to be possible in the past [14], [31], [50]. We shall now summarize some techniques for this.

Consider the $M$-band QMF bank of Fig. 29(a) again. As seen in Section VII-A, there are $M-1$ alias components in $\hat{x}(n)$, and these components are removed if and only if (64) holds. If perfect reconstruction is also desired, we have to force $T(z)$ to be a delay. It remains to solve for the synthesis filters $F_{k}(z)$ in (64). Once again we wish to avoid inversion of the alias-component matrix. So we lean on the polyphase representation of Fig. 29 (b), which is completely equivalent to Fig. 29(a).

As we did in section VI-B, we can choose $\boldsymbol{R}(z)=\boldsymbol{E}^{-1}(z)$ to obtain perfect reconstruction. To avoid inversion of $\boldsymbol{E}(z)$, we wish to force it to be lossless so that $\tilde{E}(z)=c E^{-1}(z)$. This means that the choice of $\boldsymbol{R}(z)$, as in (51), will ensure the PR property. Assuming that $H_{k}(z)$ (and hence $E(z)$ ) are FIR, this also ensures that $\boldsymbol{R}(z)$, and hence $F_{k}(z)$ are FIR. Moreover, this choice of $\boldsymbol{R}(z)$ is equivalent to choosing the synthesis filter coefficients as [31]

$$
f_{k}(n)=\alpha h_{k}^{*}\left(n_{0}-1-n\right), \quad 0 \leq k \leq M-1
$$

where $n_{0}$ is the length of the longest analysis filter, and $\alpha$ is a nonzero constant which can be scaled to be unity.

It remains to show how $\boldsymbol{E}(z)$ can be forced to be lossless. In the $M=2$ case, we had the factorization result (60) for lossless matrices. Similar factorization theorems have also been developed [62]-[64] for the $M \times M$ case. A structure has been developed in [64] for real coefficient FIR lossless transfer matrices based on a state-space approach. The structure is such that every $M \times M$ real coefficient FIR lossless system can be realized using this structure. Conversely, regardless of the values of the parameters in the structure (which are angles of rotation), the transfer matrix remains (FIR and) lossless. The development in [64] is based on the discrete-time lossless bounded real lemma [81] which relates the losslessness of $\boldsymbol{E}(z)$ to the state-space description (see end of section I for state-space notations). According to [81], a stable transfer matrix $\boldsymbol{E}(z)$ is lossless if and only if there exists a structure (with minimum number of delays) such that the system matrix

$$
R=\left[\begin{array}{ll}
A & B \\
C & D
\end{array}\right]
$$

is unitary. A complete parameterization of all lossless matrices of a given degree can therefore be obtained merely by parameterizing all unitary matrices of a given size. The development in [64] makes further use of the fact that $\boldsymbol{E}(z)$ is FIR, so that $\boldsymbol{A}$ has all eigenvalues equal to zero. Because of the unitariness of the $\boldsymbol{R}$ matrix, the structures in [64] are also said to be "orthogonal filter structures."

The only disadvantage of the structure in [64] is that the parameters in the structure are sines and cosines of angles. If we wish to optimize the analysis filter responses by optimizing these angles, then the procedure involves several computations of sines and cosines per iteration, which results in a very slow optimization procedure.

It is possible to obtain a more convenient characterization of FIR lossless matrices that is free from cosines and sines. The following result is proved in [62] and [63] and works for the real as well as complex coefficient case.

Theorem. An $M \times M$ causal FIR transfer matrix $\boldsymbol{E}(z)$ of degree $K$ is lossless if and only if it can be expressed in the form

$$
E(z)=V_{K}(z) V_{K-1}(z) \cdots V_{1}(z) H_{0}
$$

where $V_{n}(z)$ are $M \times M$ matrices of the form

$$
\boldsymbol{V}_{n}(z)=\left[\boldsymbol{I}-\boldsymbol{v}_{n} \boldsymbol{v}_{n}^{\dagger}+z^{-1} \boldsymbol{v}_{n} \boldsymbol{v}_{n}^{\dagger}\right]
$$

in which $v_{n}$ are $M \times 1$ column vectors of unit norm, and $\boldsymbol{H}_{0}$ is an $M \times M$ constant unitary matrix.

The term "degree" in the preceding statement indicates the smallest number of scalar delays (i.e., $z^{-1}$ elements) required to implement the system. It can be shown [62], [63] that the form (76) for $V_{n}(z)$ with unit-norm $v_{n}$ automatically guarantees that $\boldsymbol{V}_{n}(z)$ is (causal, FIR, and) lossless of degree one. If we write down the state-space representation of the system implemented as in (75), the system matrix $\boldsymbol{R}$ turns out to be unitary [82]. In other words, the structure for $\boldsymbol{E}(z)$ obtained in this way is an "orthogonal" structure in the same sense as those in [64] are. This property is important because it implies certain useful properties for finite wordlength implementations, such as automatic $\mathcal{L}_{2}$ scaling of internal nodes, and reduced round-off noise [83].

It is important to note that for the case of real coefficient FIR lossless matrices, the unit-norm vectors $v_{n}$ in (76) and the unitary matrix $\boldsymbol{H}_{0}$ in (75) are real.

It is possible to optimize the unit-norm vectors $\boldsymbol{v}_{n}$ in (76) and the unitary matrix $\boldsymbol{H}_{0}$ in (75) such that the sum of stopband energies of the analysis filters $H_{k}(z)$ is minimized. This issue is handled in much greater detail in [63]. In fact, it is possible to completely avoid rotation angles in the expression (75) (and this helps to speed up the optimization of the analysis filter responses) because the unitary matrix $\boldsymbol{H}_{0}$ can also be expressed as a product of $M-1$ matrices of the form $\left[\boldsymbol{I}-2 \boldsymbol{w}_{n} \boldsymbol{w}_{n}^{\dagger}\right]$ (where $\boldsymbol{w}_{n}$ are unit-norm column vectors), followed by a diagonal unitary matrix. The factor $[\boldsymbol{I}-$ $\left.2 \boldsymbol{w}_{n} \boldsymbol{w}_{n}^{\dagger}\right]$, which is a unitary matrix, is the well-known Householder form.

The degree-one lossless building blocks described by (76) can be implemented with $2 M$ multipliers and one scalar delay (see Fig. 33). For the real coefficient case, $\boldsymbol{H}_{0}$ can be

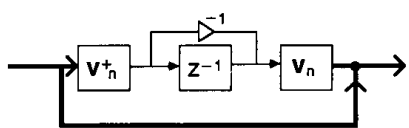

Fig. 33. Implementation of degree-one $M \times M$ FIR lossless building block using $2 M$ multipliers and one scalar delay.

implemented using $\left({ }_{2}^{M}\right)$ planar rotation angles (see [64] and [84]), each of which is equivalent to four real multiplications. The matrix $\boldsymbol{H}_{0}$ then requires $4\left(\begin{array}{c}M \\ 2\end{array}\right)$ multiplications so that the total number of real multipliers in a real-coefficient FIR analysis bank with lossless $E(z)$, as in (75), is equal to $2 M K$ $+2 M(M-1)$.

Robustness of Perfect Reconstruction Under Coefficient Quantization: Recall that as long as the polyphase matrix of the analysis bank has the form (75), where $v_{n}$ are unit-norm 
vectors and $\boldsymbol{H}_{0}$ is unitary, the filter bank of Fig. 29(a) has perfect reconstruction provided the synthesis filters are chosen as in (74). The unit-norm requirement of $v_{n}$ and unitariness of $\boldsymbol{H}_{0}$ are essential to ensure losslessness of $\boldsymbol{E}(\boldsymbol{z})$ (and hence perfect reconstruction). Now suppose that we actually implement the analysis and synthesis banks in polyphase form (Fig. 29(b)) with $\boldsymbol{R}(\mathrm{z})$ related to $\boldsymbol{E}(\mathrm{z})$ as in (51) (i.e., $f_{k}(n)$ related to $h_{k}(n)$ as in (74)). In particular, assume that $\boldsymbol{E}(z)$ is implemented as in (75), with building blocks as in Fig. 33. In practice we have to quantize the components of $v_{n}$ appearing in the building block. The norm of the quantized $v_{n}$ is not unity any more. If we do not exercise additional care, perfect reconstruction property will be lost because of this. However, here is a simple trick which overcomes this problem: instead of using the building blocks (76) use the denormalized building blocks

$$
\boldsymbol{U}_{n}(\mathrm{z})=\left[\boldsymbol{u}_{n}^{\dagger} \boldsymbol{u}_{n} \boldsymbol{l}-\boldsymbol{u}_{n} \boldsymbol{u}_{n}^{\dagger}+z^{-1} \boldsymbol{u}_{n} \boldsymbol{u}_{n}^{\dagger}\right]
$$

where $u_{n}$ is the quantized version of the unit-norm vector $\boldsymbol{v}_{n}$. The modified building blocks are lossless, i.e., $\tilde{U}_{n}(z) \boldsymbol{U}_{n}(z)$ $=a_{n} I$ for some scalar $a_{n}>0$. Next, in practice, the elements of the unitary matrix $\boldsymbol{H}_{0}$ in (75) should also be quantized. Recall that $\boldsymbol{H}_{0}$ can be expressed as a product of the matrices $\left[\boldsymbol{I}-2 \boldsymbol{w}_{n} \boldsymbol{w}_{n}^{\dagger}\right]$ (and a diagonal matrix). If we replace each of these with $\left[u_{n}^{\dagger} u_{n} \boldsymbol{I}-2 \boldsymbol{u}_{n} \boldsymbol{u}_{n}^{\dagger}\right]$, where $\boldsymbol{u}_{n}$ is a quantized version of $w_{n}$, then the resulting product is still unitary (but denormalized). The overall result of these replacements is that, $\boldsymbol{E}(z)$ is replaced with $\boldsymbol{c} \boldsymbol{E}(z)$, where $c$ is some positive scalar. The losslessness property of the polyphase matrix is therefore preserved under quantization, and so is the perfect reconstruction property of the filter bank!

Other Quantization Effects: In general, quantization of the filter coefficients in the analysis bank results in degradation of the analysis filter responses. Because of the lossless property of $\boldsymbol{E}(z)$, it is, however, possible to reduce the passband sensitivity of the responses $\left|H_{k}\left(\mathrm{e}^{j \omega}\right)\right|$ with respect to coefficient quantization (as discussed in [42], [43], and [60]). It should, however, be noted that in subband coding applications, passband sensitivity of the analysis filters is not very crucial. It is more important to have good rejection of adjacent-band signals (i.e., good stopband attenuations) so that the subband features can be exploited during the coding process. Simulation results in [65] show that the stopband sensitivity of the analysis filters with respect to the coefficients of the lossless matrix $F(z)$ are acceptably low in practice. It is also desirable to have perfect reconstruction in spite of coefficient quantization, which in turn can be accomplished as described in the preceding.

Roundoff Noise and Coding Noise: The FIR perfect reconstruction system implemented in terms of the lossless matrices $\boldsymbol{E}(z)$ and $\boldsymbol{R}(z)$ is also subject to roundoff noise errors, due to internal signal quantization. This has been analyzed in [65] for the two-channel case. Because of the lossless nature of the building blocks, the noise gain from the location of the noise source to the location of $\hat{x}(n)$ does not exceed unity. The output noise variance is therefore well under control. See [65] for more quantitative details. A second (and major) source of error arises from the fact that the subband signals (outputs of the analysis filters $\left.H_{k}(z)\right)$ are coded prior to transmission. The analysis of this noise is rendered difficult because the coding noise sources from adjacent channels are not necessarily uncorrelated and, moreover, each noise source can be colored.
IIRQMF Banks with Lossless $E(\mathrm{z})$ : If we replace each delay element $z^{-1}$ in (75) with a degree-one all-pass function $\left(\alpha_{n}^{*}+z^{-1}\right) /\left(1+\alpha_{n} z^{-1}\right)$ (where $\left|\alpha_{n}\right|<1$ for stability), then $\boldsymbol{E}(z)$ becomes an IIR lossless system. Moreover, every degree- $K M \times M$ IIR lossless system can be realized in this manner [85]. In the FIR case with lossless $E(z)$, we had to choose $\boldsymbol{R}(\mathrm{z})$, as in (51), to obtain perfect reconstruction. In the IIR case this cannot be done because $\tilde{E}(z)$ will have poles outside the unit circle. To overcome this problem we proceed as follows: we write $\boldsymbol{E}(z)=\boldsymbol{P}(z) / d(z)$, where $d(z)$ is the least common multiple of the denominators of the entries of $\boldsymbol{E}(z)$, and $\boldsymbol{P}(z)$ is the matrix of correspondingly adjusted numerator polynomials. We then choose $\boldsymbol{R}(z)=z^{-K} \tilde{\boldsymbol{P}}(z) / d(z)$ so that $\boldsymbol{R}(z)$ (and hence the synthesis filters) are guaranteed to be stable. With this choice we have $\boldsymbol{R}(z) \boldsymbol{E}(z)=z^{-\kappa}[\tilde{d}(z) l$ $d(z)] I$, so that the distortion $T(z)$ in (29) is all-pass. Summarizing, there is no amplitude distortion, only phase distortion. A second method based on the determinant of $E(z)$ is indicated in page 491 of [31]. These ideas work for arbitrary $M$.

FIR Design Examples: We shall now present design examples of FIR perfect reconstruction systems. For a threechannel system, Fig. 34(a) shows an example of the responses $\left|H_{k}\left(\mathrm{e}^{j \omega}\right)\right|$ of the three analysis filters. These were

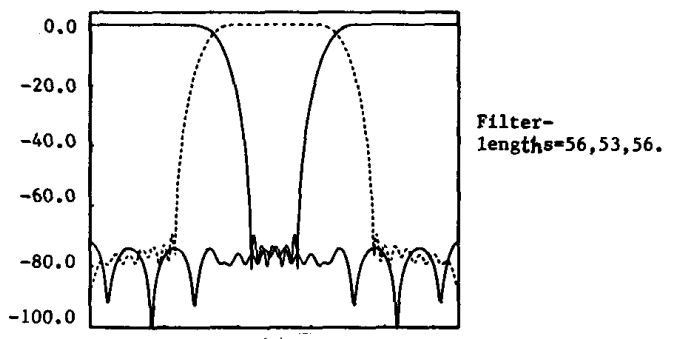

(a)

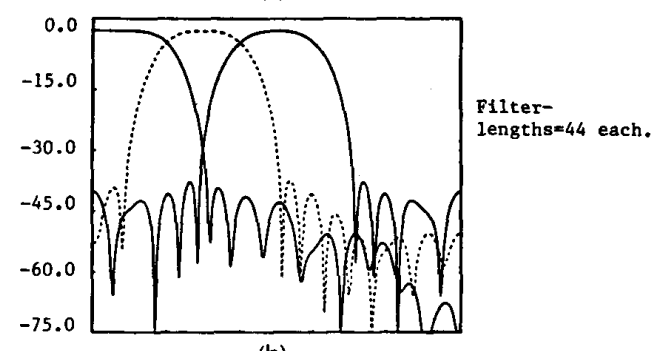

(b)

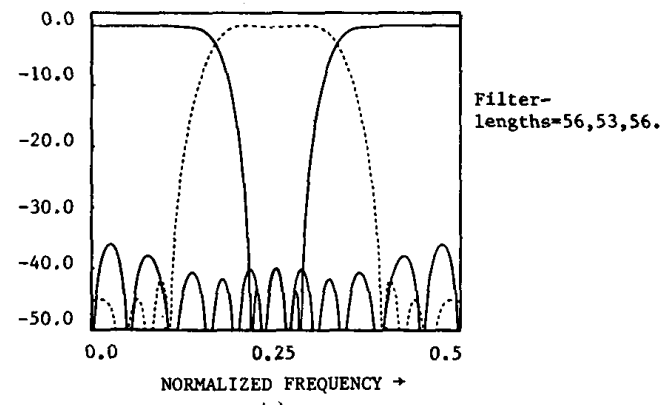

(c)

Fig. 34. Examples of magnitude responses in $\mathrm{dB}$ for analysis filters of various FIR perfect reconstruction systems. (a) Three-band analysis bank. (b) Five-band analysis bank. (c) Three-band linear-phase analysis bank. 
designed by constraining $E(z)$ to be lossless as just described, and optimizing the parameters of $\boldsymbol{v}_{n}$ and $\boldsymbol{H}_{\mathbf{0}}$. The optimization was carried out using appropriate software in [86]. Initialization of the optimization was done as described in [63]. To obtain perfect reconstruction, the FIR synthesis filters were obtained as in (74) with $n_{0}=56$. As a second example, Fig. 34(b) shows the responses of the analysis filters $H_{0}(z), H_{1}(z)$, and $H_{2}(z)$ for the $M=5$ case. The remaining two filters are given by $H_{3}(z)=H_{1}(-z)$ and $H_{4}(z)=H_{0}(-z)$, and are therefore not shown (this relation is not necessary for perfect reconstruction, nor is it automatic; see [64]). The FIR matrix $E(z)$ was again constrained to be lossless. Once again, if synthesis filters are chosen according to (74) with $n_{0}=44$, we have perfect reconstruction, because of the losslessness of $\boldsymbol{E}(z)$ which was forced during the design of $H_{k}(z)$ 's. The table of filter coefficients for these two examples can be found in [63] and [64], respectively. Finally, Fig. 34(c) shows the analysis filter responses for a three-channel QMF bank, where the analysis filters have linear phase; the matrix $\boldsymbol{E}(z)$ is not lossless here, but still has a determinant equal to a delay. The table of filter coefficients for this example can be found in [87].

Methods Based on Tree-Structures: When $M$ is a power of two, we can obtain the QMF bank by repeated use of the two-channel QMF bank. See [54] for details. If the two-channel building block is designed to have the PR property, then so does the $M$ channel tree-structured design. Since the design of the filters $H_{0}(z)$ and $H_{1}(z)$ is easier than a direct $M$-band design, tree structures are often considered attractive. However, for a given $M$, tree structures are not optimal in terms of filter lengths and result in greater group delay of the overall QMF system, compared to nontree designs. Such group delays are of concern in long-distance communication links, where echos are possible.

Methods Based on Approximations: Some authors have developed methods for designing the filters $H_{k}(z)$ such that the three distortions (ALD, AMD, and PHD) are approximately eliminated. Notable among these are [88] and [89]. These are based on the design of an appropriate optimized prototype filter, from which the other filters can be derived using a modified form of cosine modulation. The approximate designs are sometimes less expensive to implement than the perfect ones.

Price Paid for Perfect Reconstruction: It is sometimes assumed that one must pay a high price in terms of design complexity, to achieve perfect reconstruction in FIR QMF banks. This, however, is not true. The PR property often admits the representation of the analysis bank in terms of lattice structures, which are surprisingly efficient. If a PR system is implemented in lattice form, it is often not more expensive than an approximate design with comparable attenuations for the analysis filters. See section VI-E and Tables 1-3 of this paper; also see [90, pp. 701-703] for further quantitative remarks.

As a further demonstration of this claim, consider the FIR perfect reconstruction example in Fig. 34(c). The analysis filters here have linear phase. A design example is presented in [87] for the $M=3$ case, where the filters $H_{0}(z)$, $H_{1}(z)$, and $H_{2}(z)$ have lengths 56,53 , and 56 , respectively. The entire analysis bank of three filters, if implemented using the lattice structure in [87], requires a total of only $8 \mathrm{MPUs}$ and 11 APUs!

Let us now count the total number of multiplications in the $M$ band FIR analysis bank with lossless polyphase matrix $\boldsymbol{E}(z)$ implemented as in (75). Assuming the coefficients of the filters to be real (so that $\boldsymbol{v}_{n}$ and $\boldsymbol{H}_{0}$ are real), we have a total of $2 M K+2 M(M-1)$ multipliers for the analysis bank. With $E(z)$ as in (75), the analysis filters have maximum length $N$ $=K M+M$, so that the number of multipliers becomes $2(N$ $-1)+2(M-1)^{2}$. Recognizing that these multiplications can be performed at the lower rate (by moving the decimators to the left of $E(z)$ in Fig. 29(b)), the number of MPUs for the analysis bank is equal to $2(N-1) / M+2(M-1)^{2} / M \approx 2(N$ - 1) $/ M+2 M$. In comparison, consider the uniform DFT analysis bank of Fig. 21, which we found to be very efficient in section IV. Assuming that the analysis filters have length $N$ each, the total number of multiplications is equal to $N$ plus the cost of IDFT. The number of MPUs is equal to $N / M$ plus the DFT cost, which depends on $M$. For $N \gg M$ we see that the perfect reconstruction FIR system with lossless $\boldsymbol{E}(z)$ implemented as in (75) is only about two times more expensive than the uniform DFT filter bank with same filter orders! In fact, if we impose certain symmetry conditions among the analysis filters $H_{k}(z)$ in the perfect reconstruction system (as elaborated in [90]), then the number of MPUs for the perfect reconstruction system is reduced by a factor of two, so that it becomes equal to that of the uniform DFT polyphase analysis bank. Summarizing, the computational complexity of the FIR perfect-reconstruction analysis bank based on lossless polyphase matrices can be made comparable to that of the uniform DFT polyphase analysis bank for same $N$, whenever $N \gg M$.

Unsuitability of the Uniform DFT QMF Bank for Perfect Alias Cancellation: The uniform DFT FIR analysis bank shown in Fig. 21 is computationally very efficient, and is well suited for spectrum analysis. However, it is usually not well suited for QMF bank applications requiring perfect reconstruction (hence perfect alias cancellation in particular). This can be seen by referring to [12], which derives the necessary and sufficient conditions on the FIR synthesis filters for alias cancellation in this case. It turns out that the synthesis filters typically have much higher orders than the analysis filters, resulting in a much more expensive synthesis bank. In contrast to this, the perfect reconstruction FIR QMF bank based on general lossless FIR E(z) (rather than on uniform DFT analysis bank) is such that each synthesis filter has the same length as the corresponding analysis filter (see (74)). The overall cost of the complete analysis/synthesis system is therefore lower.

Alias-component matrix, polyphase component matrix, and interrelations: It can be shown [31] that the $M \times M$ matrix in (64) is lossless if and only if the polyphase component matrix $E(z)$ is lossless. So by forcing $E(z)$ to be lossless, we make the inversion of (64) simple; in fact, closedform expressions for $f_{k}(n)$ as in (74) are obtained.

For any $M$-band maximally decimated FIR QMF bank, consider the following three properties: a) losslessness of $E(z)$; b) the relation (74); and c) perfect reconstruction property (32). It can be shown [31] that any two of these imply the third. For example, if a QMF bank satisfies (74) and has perfect reconstruction property, then $E(z)$ must be lossless. Similarly, if $\boldsymbol{E}(z)$ is lossless, then we have perfect reconstruction if and only if (74) holds. Note, however, that neither losslessness of $E(z)$ nor the relation (74) are, by themselves, necessary or sufficient conditions for perfect reconstruction. 
Notice that in Fig. 29(b) if $E(z)$ is replaced with the $M \times$ $M$ DFT matrix, it is equivalent to performing an orthogonal (rather unitary) transformation on blocks of the input. A more general type of orthogonal transformation called the "lapped orthogonal transformation" (LOT) has recently been used in image processing applications [91]. Interestingly enough, the LOT has been developed quite independently (see [91] and references therein) but turns out to be an analysis bank with lossless $\boldsymbol{E}(z)$ of low degree. See [92], where the authors have established the link between LOT and paraunitariness; also see [93].

\section{iX. Unconventional Applications}

In this section we outline some unconventional applications of multirate filter banks in signal processing. These include new sampling theorems, new techniques for efficient quantization of filter coefficients, and adaptive filtering in subbands.

\section{A. New Sampling Theorems Based on the QMF Principle} and Polyphase Decompositions

The sampling theorem for continuous-time signals has been well known for several decades [94], [95]. The earliest known version says that if a continuous-time signal $x_{a}(t)$ is bandlimited to $-\sigma<\Omega<\sigma$, it can be reconstructed from equally spaced samples $x(n)=x_{\mathrm{a}}(n T)$ provided the sampling frequency $2 \pi / T$ exceeeds the so-called Nyquist rate $\theta \triangleq 2 \sigma$. Under this condition, the transform $X\left(e^{j \omega}\right)$ is a periodic repetition of $X_{a}(j \Omega)$ properly scaled, as shown in Fig. 35. Quan-
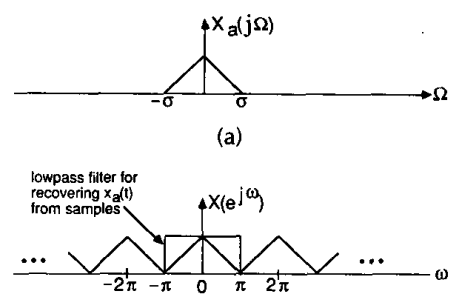

(b)

Fig. 35. Transform of $x_{a}(t)$ and transform of sampled version $x_{a}(n T)$. (a) Signal recovery. (b) Low-pass filtering.

titatively,

$$
X\left(\mathrm{e}^{j \omega}\right)=\frac{1}{T} \sum_{k=-\infty}^{\infty} X_{a}\left(j \Omega-\frac{j 2 \pi k}{T}\right), \quad \omega=\Omega T .
$$

The signal $x_{a}(t)$ can be recovered from the samples $x(n)$ merely by low-pass filtering the impulse train $\Sigma_{n=-\infty}^{\infty} x(n)$ $\delta(t-n T)$, which results in elimination of the unwanted copies of the basic spectrum $X_{a}(j \Omega)$ in Fig. 35(b). Notice that the low-pass filter required is an ideal filter which is IIR, noncausal, and unstable [7], [8].

Several generalizations of this theorem are known [96]. Perhaps the earliest one, called the derivative sampling theorem, says that if $x_{a}(t)$ and $d x_{a}(t) / d t$ are available (for example, the position and velocity of an aircraft), then uniformly spaced samples of these two waveforms, taken at half the Nyquist rate, are sufficient to retain all the information. In other words, the signal $x_{a}(t)$ can be recovered from these samples. Once again, the use of ideal filters is required in the recovery process. A natural extension of this theorem is that if $x_{a}(t)$ and the first $M-1$ derivatives are available (such as the position, velocity, acceleration, jerk, etc. of an aircraft), then each of these can be uniformly sampled at the rate $\theta / M$ without losing information. No simple closed-form expressions are available, however, for the reconstruction of $x_{a}(t)$ from these generalized samples.

The second family of generalizations is the class of nonuniform sampling theorems [96]. The basic idea is that the samples of $x_{a}(t)$ can be spaced in an entirely nonuniform manner (Fig. 36) provided the sampling rate averaged over

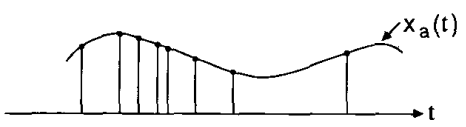

Fig. 36. Demonstration of nonuniform sampling.

a sufficiently long period exceeds $\theta$. More precise statement of this result can be found in [96] and references therein. This result is called the nonuniform sampling theorem, and less formally, the "folk theorem." The knowledge of this result seems to date back [97] to the days of Cauchy (1841!). An extreme case of the folk theorem is the assertion that a bandlimited signal is completely determined everywhere if all its past samples, taken at twice the Nyquist rate, are available! The reconstruction of $x_{a}(t)$ from the nonuniformly spaced samples is, in general, very involved [98], [99].

However, if we take a second look at these sampling theorems from a discrete-time viewpoint, the insight obtainable is rewarding. For example, let $x(n)$ be a bandlimited sequence, i.e., a sequence whose transform $X\left(\mathrm{e}^{j \omega}\right)$ vanishes for $\omega_{\mathrm{c}} \leq|\omega| \leq \pi$, with $\omega_{\mathrm{c}}<\pi$. An example with $\omega_{\mathrm{c}}=2 \pi / 3$ was seen in Fig. 8(b). It is clear that we can construct a new sequence $y(n)$ with a lower rate, without losing information. Fig. 8(a) shows how to do this in terms of the fractional decimator circuit $(L=2, M=3)$. We know that if $H(z)$ is ideal low-pass with cutoff $2 \pi / 3, Y\left(e^{j \omega}\right)$ is a stretched version of $X\left(e^{j \omega}\right)$ by the factor $3 / 2$.

This, however, is not the only way to reduce the sampling rate. A simpler way to compress $x(n)$ in Fig. 8 (b) would be to divide the time index into segments of length three and retain two out of three samples in each segment. This is equivalent to nonuniform sampling of a hypothetical underlying continuous-time signal. From this nonuniformly decimated version $y(n)$, is it possible to recover $x(n)$ ? The answer is yes, as we would expect because of our knowledge about the "folk theorems."

The main point we wish to make [30] is that this problem can be stated in the form of a QMF bank design problem. With such a restatement, the algorithm for reconstruction of $x(n)$ from $y(n)$ is seen to be a simple synthesis-bank design problem, which actually can be carried out in practice with FIR filters. To demonstrate these ideas, consider the example of Fig. 8(b) again. We wish a data rate reduction of $3 / 2$. This will be accomplished by retaining two out of every consecutive segment of three samples. Fig. 37 shows a way to mechanize this subsampling based on multirate building blocks. The samples retained in the process are those numbered $\cdots-4,-3,-1,0,2,3,5,6,8,9, \cdots$. Consider now the $M$-channel maximally decimated QMF bank of Fig. 29(a) 


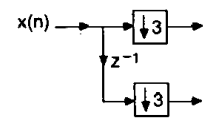

Fig. 37. Mechanization of nonuniform subsampling process.

with $M=3$, and let the analysis filters be $H_{0}(z)=1, H_{\uparrow}(z)=$ $z^{-1}$, and $H_{2}(z)=0$. The analysis bank then resembles Fig. 37. Question: how do we design the synthesis filters $F_{0}(z)$ and $F_{1}(z)$ such that $\hat{x}(n)$ is equal to $x(n)$ ? This problem can be formally solved using the matrix description of section VII-A. The results derived in [30] show that the required synthesis filters are

$$
\begin{aligned}
F_{0}(z)=z^{-1}\left[1-c+s G_{H}(z)\right] G_{L}(z) \\
F_{1}(z)=\left[1-c-s G_{H}(z)\right] G_{L}(z)
\end{aligned}
$$

where $C_{L}(z)$ is an ideal low-pass filter with cutoff $\omega_{c}=$ $2 \pi / 3$ and $G_{H}(z)$ is an ideal Hilbert transformer [16], [17], i.e., $G_{H}\left(e^{j \omega}\right)=j s g n[\omega]$. The claim is that the choice (77) results in $\hat{x}(n)=x(n-1)$. In practice, the ideal responses can be approximated with linear-phase FIR filters with any desired accuracy. See [30] for design examples of this type. Extension of these results for the multidimensional case can be found in [101].

A more complete analysis of this type of reconstruction filter banks has been done in [101]. For the case of general $L$ and $M$, the ideal solution for the reconstruction filters turns out to be such that each filter $F_{k}(z)$ is a multilevel filter. (Multilevel filters are described in section IX-D of this paper.) This means that the response $F_{k}\left(e^{j \omega}\right)$ is a piecewise constant with $M$ (possibly complex) levels in the region $0 \leq \omega<2 \pi$. It turns out that all the $M$ filters $F_{k}(z)$ can in practice be implemented by implementing a single $M$ th band low-pass filter in polyphase form, and obtaining fixed linear combinations of the outputs of the polyphase components. Consequently, the price paid for the set of reconstruction filters is equal to the price of a single $M$ th band low-pass filter plus the cost of implementing a constant $M \times L$ matrix. Details can be found in [101].

The advantage of these compression techniques based on nonuniform subsampling is that the compression is computationally very simple. The only price paid is for the implementation of filters $F_{k}(z)$ at the reconstruction stage. These techniques are attractive when a transmitter has low computational capability, while the receiver (e.g., a ground station) has more resources for signal retrieval.

The second type of sampling theorem for sequences is somewhat similar to the derivative sampling theorems. Let $x(n)$ be some sequence, not necessarily bandlimited, which we wish to transmit (or just store). Let us define the first difference as $x_{1}(n)=x(n)-x(n-1)$. Suppose, instead of transmitting $x(n)$ we transmit twofold decimated versions of $x(n)$ and $x_{1}(n)$. (We will soon talk about advantages of doing this). From the received signals is it possible to get back $x(n)$ for all $n$ ? The answer is yes, as the reader might have guessed. In fact, more is true. If we are given $x(n)$, and the first $M-1$ differences $x_{k}(n), 1 \leq k \leq M-1$, we can transmit the $M$-fold decimated versions of these $M$ sequences, and eventually reconstruct $x(n)$ at the receiver. Moreover, such reconstruction can be done perfectly with FIR filters. These filters, in addition, are multiplierless!
This result can be derived by recognizing that the problem can be posed as a filter bank design problem (Fig. 29(a)), where the analysis filters are $H_{k}(z)=\left(1-z^{-1}\right)^{k}$, which corresponds to the difference operators. The task is to design the synthesis filters $F_{k}(z), 0 \leq k \leq M \leq 1$ such that $\hat{x}(n)=$ $x\left(n-n_{0}\right)$. Even though the solution for $F_{k}(z)$ is not obvious by looking at Fig. 29(a), it becomes quite tractable once we redraw the structure as in Fig. 29(b). The polyphase matrix $\boldsymbol{E}(z)$ in this case turns out to be a constant lower triangular matrix which is its own inverse [30, Lemma 3.1]. With $\boldsymbol{R}(z)$ chosen as $E^{-1}(z)$ for perfect reconstruction, we then have $\boldsymbol{R}(z)=\boldsymbol{E}(z)$, and the synthesis filters given by (65) are evidently FIR. As an example, consider the $M=3$ case, so that

$$
\boldsymbol{E}(z)=\boldsymbol{R}(z)=\left[\begin{array}{rrr}
1 & 0 & 0 \\
1 & -1 & 0 \\
1 & -2 & 1
\end{array}\right]
$$

Using (65), the synthesis filters turn out to be

$$
\begin{gathered}
F_{0}(z)=1+z^{-1}+z^{-2}, \quad F_{1}(z)=-z^{-1}-2, \\
F_{2}(z)=1 .
\end{gathered}
$$

For sequences of certain types, difference sampling theorems offer an advantage. Consider, for example, a signal $x(n)$, each of whose samples is a 16-bit number. Clearly it cannot be bandlimited (because it is quantized). But the first difference $x_{1}(n)$ can be quite small (see Fig. 38 for dem-

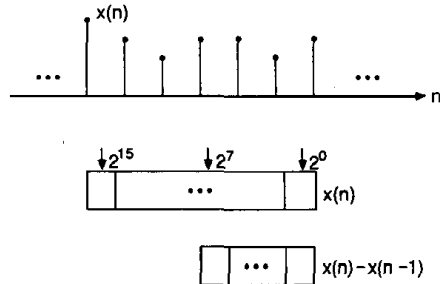

Fig. 38. Binary register patterns for hypothetical sequence and its first difference.

onstration), and so representable by, e.g., 8 bits/sample. If we transmit decimated versions of $x(n)$ and $x_{1}(n)$ with 16 and 8 bits per sample, then the average data rate is now 12 bits per sample, which is an improvement.

\section{B. Efficient Quantization of Filter-Coefficients Using the} Generalized Polyphase (GPP) Decomposition

In section III-B we saw that the subband coding technique can be used for efficient representation of a sequence whose spectral energy is very low in some frequency regions compared to others. Can we apply the same trick for efficient representation of the coefficients $h(n)$ of a digital filter? In other words, does it make sense to do subband coding of impulse responses? After all, the frequency response $H\left(\mathrm{e}^{j \omega}\right)$ in most applications does have very low energy in some frequency bands. The anticipated advantage would be that the average number of bits per sample of $h(n)$ could possibly be reduced, resulting in a more efficient implementation of $H(z)$.

The main flaw in this kind of reasoning is that subband coding of a sequence requires a filter-bank system. If the 
price paid for the filter bank outweighs the advantage gained from efficient representation of $h(n)$, we are losers in the game! As a result, the idea works only if the filter bank turns out to be a simple low-cost device. Based on intuitive reasonings we shall now derive an appropriate low-cost "QMF bank" that can be used for such applications.

To fix ideas, let $H(z)$ be a narrow-band low-pass decimation filter. It is well known [8], [17] then that the adjacent samples of the impulse response $h(n)$ are highly correlated i.e., $h(n)-h(n-1)$ is typically very small compared to the average $[h(n)+h(n-1)] / 2$. Our aim is to exploit this. Let us represent $H(z)$ in terms of its type-1 polyphase form (7). After moving the decimators (assumed to be two fold for simplicity) using the appropriate noble identity, the structure is as in Fig. 15(b). Suppose now that we insert a matrix $T$ and its inverse in Fig. 15(b), to obtain Fig. 39(a). Evidently, this does not affect $x(n)$ at all! As an example let $\boldsymbol{T}=\left[\begin{array}{ll}1 & 1 \\ 0 & -1\end{array}\right]$ so that $T^{-1}=T$ here. Noting that

$$
\boldsymbol{T}^{-1}\left[\begin{array}{l}
1 \\
z^{-1}
\end{array}\right]=\left[\begin{array}{c}
1+z^{-1} \\
-z^{-1}
\end{array}\right]
$$

and that

$$
\left[E_{0}(z) \quad E_{1}(z)\right] \boldsymbol{T}=\left[\begin{array}{lll}
E_{0}(z) & E_{0}(z)-E_{1}(z)
\end{array}\right]
$$

we can redraw Fig. 39(a) as in Fig. 39(b). The coefficients of $E_{0}(z)-E_{1}(z)$ are composed of the first difference of $h(n)$, which are expected to be very small.
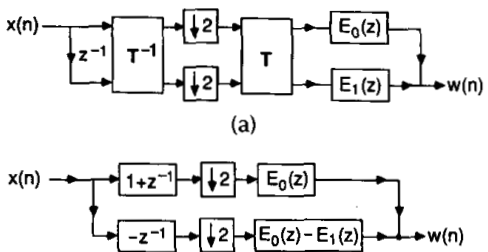

(b)

Fig. 39. New type of polyphase decomposition for filtercoding applications. (a) Implementation based on insertion of matrix $T$ and its inverse in Fig. 15(b). (b) Redrawing using equations (80) and (81).

Comparing the two equivalent structures (Fig. 15(b) versus Fig. 39(b)) for the decimation filter, we see that both have the advantage offered by the polyphase representation, namely computation at the lower rate. The additional advantage offered by Fig. 39(b) is that the lower branch contains $E_{0}(z)-E_{1}(z)$ (rather than $E_{1}(z)$ ), which can be represented with much fewer bits than $E_{0}(\mathrm{z})$.

Now we come to generalizations. Suppose we represent $H(z)$ in the $M$-component type-1 polyphase form (10). We can now insert an $M \times M$ nonsingular matrix $T$ and its inverse as in Fig. 40(a). This gives us the new representation of Fig. 40(b), where

$\left[\begin{array}{lllll}G_{0}(z) & G_{1}(z) & \cdots & G_{M-1}(z)\end{array}\right]=\left[\begin{array}{llll}E_{0}(z) & E_{1}(z) & \cdots & E_{M-1}(z)\end{array}\right] \boldsymbol{T}$

and

$$
\left[\begin{array}{c}
U_{0}(z) \\
U_{1}(z) \\
\vdots \\
U_{M-1}(z)
\end{array}\right]=I^{-1}\left[\begin{array}{c}
1 \\
z^{-1} \\
\vdots \\
z^{-(M-1)}
\end{array}\right]
$$

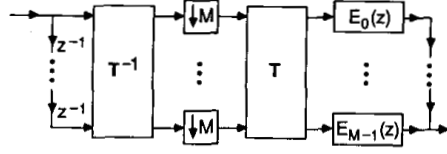

(a)

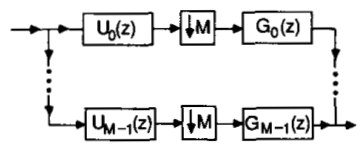

(b)

Fig. 40. Generalized polyphase (GPP) decomposition. (a) Implementation using an $M \times M$ nonsingular matrix $T$ and its inverse. (b) Redrawing using equations (82) and (83).

For example let $M=3$ and let

$$
\boldsymbol{T}=\left[\begin{array}{rrr}
1 & 1 & 1 \\
0 & -1 & -2 \\
0 & 0 & 1
\end{array}\right] \text {. }
$$

Note again that $T^{-1}=T$ here. We can then simplify Fig. 40 to Fig. 41 . In this example the difference $E_{0}(z)-E_{1}(z)$ again

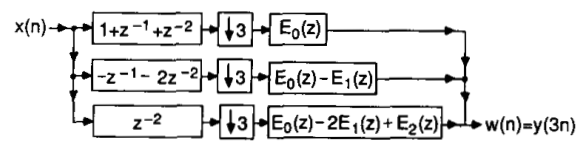

Fig. 41. Generalized polyphase implementation of decimation filter with $M=3$.

has coefficients requiring fewer bits than $E_{0}(z)$. The same is true with the second difference $E_{0}(z)-2 E_{1}(z)+E_{2}(z)$, and so on.

These specific examples are such that the columns of $\boldsymbol{T}$ correspond to the coefficients of the difference operators $\left(1-z^{-1}\right)^{k}$. This is the reason for the appearance of the differences $E_{0}(z)-E_{1}(z), E_{0}(z)-2 E_{1}(z)+E_{2}(z)$, etc. in Fig. 41. In general, if the $M \times M$ matrix $T$ has columns chosen as in the preceding, we have $\boldsymbol{T}^{-1}=\boldsymbol{T}$ (the proof follows by using Lemma 3.1 in [30]).

There are $M$ branches in Fig. 40. In general, for narrowband $H(z)$ with $T$ chosen as in the preceding, the branches have decreasing importance as $k$ grows. Thus the coefficients of $G_{k}(z)$ can be quantized to fewer and fewer bits as $k$ grows. Note that if we altogether discard every branch with $k>0$, we obtain the recently reported IFIR scheme [102] for efficient design of narrowband filters (elaborated below). The choice of $\boldsymbol{I}$ with columns equal to the coefficients of $\left(1-z^{-1}\right)^{k}$ is not necessarily the optimal choice from the viewpoint of obtaining the most efficient implementation of $H(z)$, and is used here only as a demonstration. Further study is required to determine the optimal choice of $T$ for any given $H(z)$. In any case, the representation of Fig. 40 (b) can be expressed as

$$
H(z)=\sum_{k=0}^{M-1} U_{k}(z) G_{k}\left(z^{M}\right)
$$

which we shall call the generalized polyphase (GPP) decomposition. With $U_{k}(z)=z^{-k}$ we obtain the type-1 polyphase representation (10), and $G_{k}(z)$ automatically becomes $E_{k}(z)$. 


\section{The Interpolated FIR (IFIR) Approach}

In recent years, a number of techniques have been proposed by several authors for efficient implementation of narrowband digital FIR filters [102], [103]. The IFIR approach is a simple and elegant technique for such a purpose, introduced by Neuvo et al. in [102]. Consider the design of a narrowband linear-phase FIR low-pass filter with desired response as in Fig. 42(a). The transition bandwidth $\left(\omega_{s}-\omega_{p}\right)$

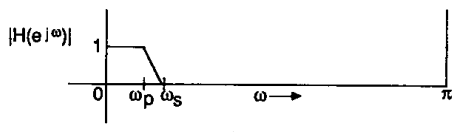

(a)

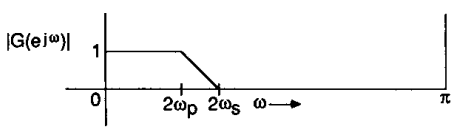

(b)

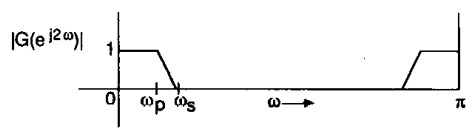

(c)

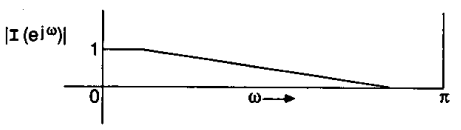

(d)

Fig. 42. Illustrating IFIR approach for narrow-band FIR design. (a) Low-pass filter with desired response. (b) Twofold stretched response. (c) Replacing each delay with two delays. (d) Removing unwanted passband by use of low-pass filter I(z)

is also narrow so that the required order is very high [17]. As an example, let the passband ripple $\delta_{1}$ and stopband ripple $\delta_{2}$ be equal. Let the stopband attenuation $A_{s}$ (defined as $-20 \log _{10} \delta_{2}$ ) be equal to $40 \mathrm{~dB}$, with $\omega_{p}=0.16 \pi$ and $\omega_{\mathrm{s}}$ $=0.24 \pi$. An equiripple design $H(z)$ then requires an order of 52 (see [16, page 80]). Instead of designing the filter to meet the response of Fig. 42(a) directly, suppose we design a linear-phase FIR filter $G(z)$ to meet the twofold stretched response in Fig. 42(b). Now the transition band is two times wider (but the peak ripples in the passband and stopband are unaltered), so that the required order is 26 . If we now replace each delay in the implementation of $G(z)$ with two delays, the response is as in Fig. 42(c). In other words, we have the desired passband and an unwanted passband. The unwanted passband can be removed by a low-pass filter $/(z)$ with response as in Fig. 42(d). Notice that the transition band of $/(z)$ is very wide so that $/(z)$ is of very low order. The overall transfer function is now $H_{1}(z)=G\left(z^{2}\right) l(z)$ and can be used in place of the direct design $H(z)$.

This indirect design approach is called the IFIR (interpolated FIR) approach for reasons described in [102]. The filter $G(z)$ is called the model filter, and $/(z)$ the "interpolator" (because I( $z)$ interpolates the impulse response of $G\left(z^{2}\right)$ [102]). The order of the IFIR design $G\left(z^{2}\right) l(z)$ is clearly higher than the direct design $H(z)$ (due to the extra order contributed by $l(z))$. However, the computational com- plexity is lower. Taking the impulse response symmetry into account, the directly designed filter $H(z)$ for the preceding specifications requires 27 multipliers. The filter $G(z)$ (and hence $G\left(z^{2}\right)$ ) requires only 14 multipliers. So the IFIR design requires about half as many multipliers (because $/(z)$ can be made practically multiplierless; see [16], [102]). If we now assume that $H(z)$ is a factor-of-two decimation filter, then a direct design of $H(z)$ would require $27 / 2 \approx 14$ MPUs (after using the polyphase trick of Fig. 15), whereas the IFIR design would require about $14 / 2=7 \mathrm{MPU}$ s plus the small overhead of implementing $I(z)$. More generally, assume the low-pass specification of Fig. 42(a) to be such that $\omega_{s} \ll \pi / M$, so that its output can be decimated by $M$ without causing aliasing. Let $K$ be the order of a direct design $H(z)$. The specification can now be stretched $M$-fold for designing the model filter $G(z)$, so that the order of $G(z)$ in the IFIR design is $K / M$. The IFIR design is therefore $H_{1}(z)=G\left(z^{M}\right) /(z)$, where $/(z)$ now eliminates $M-1$ unwanted passbands in $G\left(z^{M}\right)$. Assuming again that the overhead cost of $/(z)$ is small, we see that the MPU count is approximately as follows: $K$ MPUs for $H(z)$ without decimation; $K / M M P U s$ for $H(z)$ followed by decimation; and only $K / M^{2}$ MPUs for $H_{1}(z)$ followed by decimation (see Fig. 43). In practice, all these numbers can be divided by two because of linear-phase symmetry.

$$
x(n) \longrightarrow I(z) \rightarrow G \rightarrow
$$

Fig. 43. Implementation of decimation filter using IFIR approach.

Combining these discussions with those in section IX-B it is clear that, structurally, the IFIR scheme can also be obtained by using the GPP approach (simply retain the top branch in Fig. 40 (b) so that the system is equivalent to a decimation filter $\left.G_{0}\left(z^{M}\right) U_{0}(z)\right)$. The reader is encouraged to see [4, Ch. 5] for multistage decimation filter design techniques, which are related to the preceding idea, and which are perhaps the earliest reported techniques for efficient narrowband filter design.

\section{FIR Filters with Adjustable Multilevel Response, and} Tunable FIR Filters

In section $V$ we discussed zero-phase FIR $M$ th band filters, and mentioned a few applications. In this section we shall discuss one more application. This is in the design of multilevel digital filters. Such a filter has several passbands with each passband response typically at a different level $\beta_{k}$, as shown in Fig. 44. In practice it is also desirable to adjust

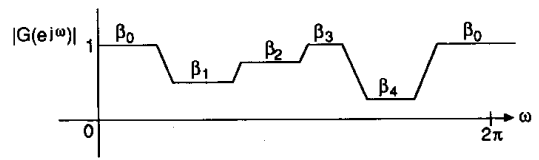

Fig. 44. Typical response of multilevel filter.

the levels $\beta_{k}$ independently by tuning a single parameter for each band, without having to redesign the entire filter. In short, we are looking for a "programmable" feature in the filter implementation.

All of this can be easily accomplished based on the poly- 
phase components of a standard low-pass zero-phase $M$ th band "prototype" filter $H(z)$ with frequency response as in Fig. 45(a). Consider the new transfer function

$$
G(z)=\sum_{k=0}^{M-1} \beta_{k} H\left(z W^{k}\right)
$$

which is a weighted sum of the responses in Fig. 45(b). In the regions where the responses of $H\left(z W^{k}\right)$ do not overlap,

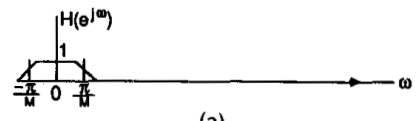

(a)

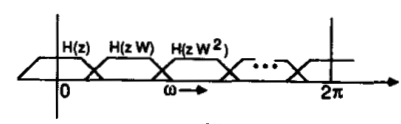

(b)

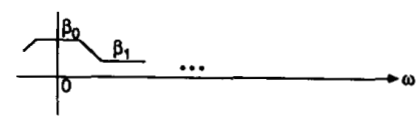

(c)

Fig. 45. Pertaining to multilevel filter design based on $M$ th band (or Nyquist) filters. (a) Prototype filter $H(z)$. (b) Responses. (c) Example of real positive monotone.

the levels are determined entirely by the $\beta_{k}$, assuming that the stopband response of $H(z)$ is small enough to be neglected. In the regions of overlap in Fig. 45(b), the response is more complicated. Consider, for example, the region around $\omega=\pi / M$. We can assume that only $H(z)$ and $H(z W)$ are significant here. From (22) (with $c=1 / M)$, we see that $\Sigma_{k=0}^{M-1} H\left(z W^{k}\right)=1$ for all $z$ so that we have

$$
H(z)+H(z W) \approx 1, \quad \text { for } \omega \text { around } \pi / M .
$$

Now consider $G(z)$ around $\omega=\pi / M$. We have, assuming that the $\beta_{k}$ are much larger than the stopband ripple of $H(z)$

$$
\begin{aligned}
G\left(e^{j \omega}\right) \approx & \beta_{0} H(z)+\beta_{1} H(z W)=\beta_{0}[H(z)+H(z W)] \\
& +\left(\beta_{1}-\beta_{0}\right) H(z W)
\end{aligned}
$$

which simplifies, in view of (87), to

$$
G\left(e^{j \omega}\right) \approx \beta_{0}+\left(\beta_{1}-\beta_{0}\right) H(z W) .
$$

The main point we wish to make is that $G\left(e^{j \omega}\right)$ in (89) is monotone in the transition region. In other words, it changes from $\beta_{0}$ to $\beta_{1}$ without any "bumps" (Fig. 45(c)), because $H(z W)$ is a real positive monotone function in the region of overlap.

Summarizing, $G\left(e^{j \omega}\right)$ is a filter with multiple pass bands, with $\beta_{k}$ denoting the $k$ th passband level and, moreover, the transition between passbands is monotone. This conclusion is a consequence of the $M$ th band property of $H(z)$. By combining the expression (86) with (21), we can simplify $G(z)$ into

$$
G(z)=\beta^{T} \boldsymbol{W}^{+} \mathbf{e}(z)
$$

where $\beta=\left[\beta_{0} \beta_{1} \cdots \beta_{M-1}\right]^{T}, \mathbf{e}(z)=\left[1 / M z^{-1} E_{1}\left(z^{M}\right) \cdots\right.$ $\left.z^{-(M-1)} E_{M-1}\left(z^{M}\right)\right]^{T}$, and $W$ is the usual $M \times M$ DFT matrix. This gives a polyphase implementation of the multiband

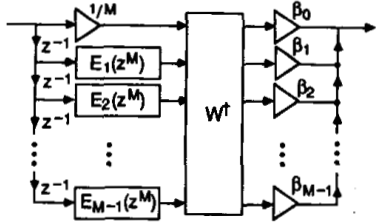

Fig. 46. Polyphase implementation of adjustable multilevel filter. Here $\beta_{k}$ represents "response level" in $k$ th band.

filter $G(z)$, whose passband levels $\beta_{k}$ are adjustable merely by tuning the multipliers $\beta_{k}$. See Fig. 46 .

Design Example: Fig, 47(a) shows the frequency response of a fifth-band ( $M=5$ ) prototype filter $H(z)$ with length $N$ $=59$, designed using the Kaiser window approach (Section $\mathrm{V}-\mathrm{A})$. Note that the responses in this figure are plotted for $0 \leq \omega \leq 2 \pi$. The stopband attenuation of $H(z)$ is about 75 dB. Fig. 47(b) shows two examples of multilevel responses. The levels used are $(0.4,1.0,0.7,0.1,0.9)$ and $(1.0,0.5,0.0$, $0.7,0.7)$, respectively. In the latter example, two adjacent levels are permitted to be equal $(=0.7)$, and the transition band between them cannot be seen. This is because, $H\left(z W^{3}\right)$ and $H\left(z W^{4}\right)$ add up to a constant very nicely in the transition band, due to the fifth-band property. This feature can be used to obtain multilevel filters with unequal bandwidths, simply by choosing $M$ to be sufficiently larger than the number of levels and appropriately combining adjacent bands.

Adjustable Cutoff FIR Filters: A second outcome of this idea is the generation of low-pass filters (and bandpass if desired), of adjustable cutoff. For example, if we choose $\beta_{0}$ $=\beta_{1}=\beta_{M-1}=1$ and the remaining $\beta^{\prime}$ s to be zero, then $G(z)$ is a real-coefficient FIR low-pass filter with cutoff $3 \pi / M$. In this way, the cutoff frequency can be "tuned" in discrete jumps. By increasing $M$, the discrete nature of the tuning can be made sufficiently close to "continuous tuning" in practice.

\section{E. Adaptive Filtering in Subbands}

Adaptive filters and their applications in signal processing such as equalization, echo cancellation, etc. are well known [104]-[108]. An important issue in the implementation of adaptive filters is the rate at which the filter coefficients converge to a solution. This rate in turn depends on the statistical properties of the input signal. To make discussions easy, let the signal be wide sense stationary with power spectrum $S(\omega)$. Let $s_{M}$ and $s_{m}$ represent the maximum and minimum values of $S(\omega)$ and let $r=s_{M} / s_{m}$. Clearly $1 \leq$ $r \leq \infty$. As a rule of thumb, we can say that the convergence is slow for large $r$ [104].

The use of band-splitting in adaptive filters was suggested first in [106]. It should be noted that frequencydomain adaptation algorithms [107] are special cases of this idea. The idea of decimating in each subband before adaptation is treated in [108]. If the bandsplitting filters were ideal bandpass filters, then the ratio $r$ for each subband will be smaller than that for the original signal. This leads to faster convergence in each subband. It should be remembered however, that in practice the filters $H_{k}(z)$ are not ideal and there is aliasing in each subband due to decimation. The effect of this on the ratio $r$ in each subband requires further study. 


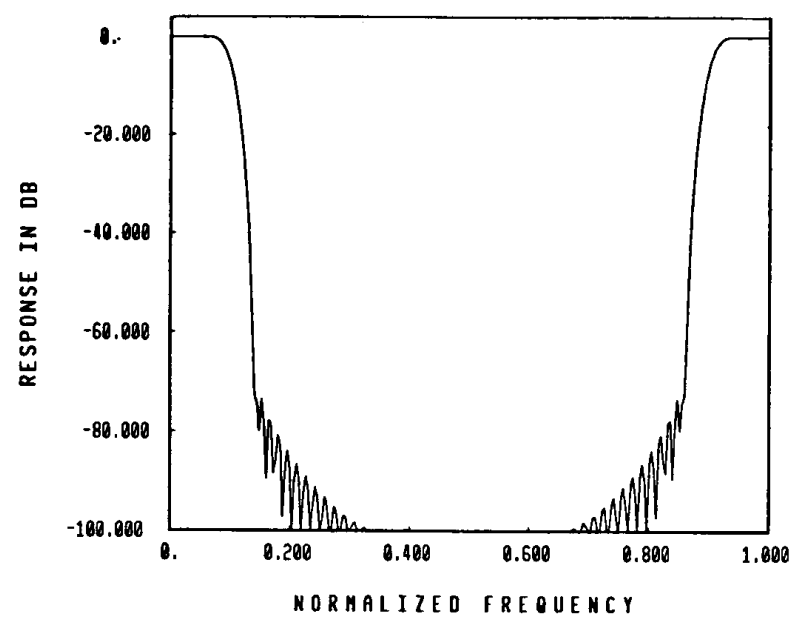

(a)

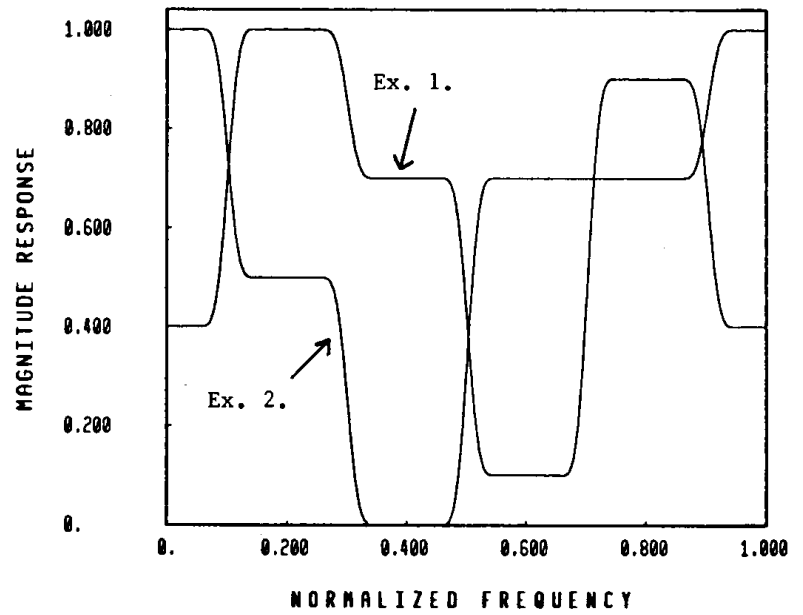

(b)

Fig. 47. Design example for adjustable multilevel FIR filters. (a) Response of prototype fifth band filter of length 59. (b) Two examples of multilevel design, obtained using polyphase network of Fig. 46.

\section{Multirate Processing of Multidimensional. Signals}

Multidimensional signals [109] are functions of more than one variable. These are denoted by notations such as $x\left(n_{1}\right.$, $n_{2}, \cdots, n_{M}$ ) and abbreviated as $x(n)$. (We also abbreviate two-dimensional as " $2 \mathrm{D}^{\prime \prime}$ and multidimensional as "MD.") It is necessary to manipulate and process such signals in applications such as video, and in radar and sonar signal processing. In this section we shall be content with pointing out the existing literature on multirate multidimensional signal processing.

The concept of sampling a two- (or higher) dimensional signal proves to be more tricky than in the one-dimensional case. This is because the sampling geometry can be rectangular or nonrectangular. One of the earliest references on the generalized sampling of multidimensional signals is the work by Petersen and Middleton [110], where the number theoretic concepts of lattices and reciprocal lattices were used to describe the sampling and aliasing processes. A lattice is basicaliy a collection of vectors of the form $\boldsymbol{V} n$, where $\boldsymbol{V}$ is a fixed nonsingular matrix of real numbers and $\boldsymbol{n}$ is a vector of integers. The matrix $\boldsymbol{V}$ is said to generate the lattice. A discrete-parameter MD signal $x(n)$ can be obtained from a continuous-parameter MD signal $x_{a}(t)$ by defining $x(n)=x_{a}(V n)$. In other words, $x_{a}(t)$ has been sampled at the locations of the lattice generated by $\boldsymbol{V}$. For this reason, $V$ is called the sampling matrix. The number of samples per unit area which results from this sampling is equal to the reciprocal of the determinant of $\boldsymbol{V}$. Notice that a diagonal $\boldsymbol{V}$ corresponds to rectangular sampling.

The reciprocal lattice is a lattice generated by the matrix $\boldsymbol{V}^{-T}$ and plays a key role in sampling theory. Basically, if $X_{a}(\Omega)$ and $X(\omega)$ are the multidimensional Fourier transforms of $x_{a}(t)$ and $x(n)$, respectively, then they are related by

$$
X(\omega)=\frac{1}{L} \sum_{k} X_{a}\left[\boldsymbol{V}^{-T}(\omega-2 \pi k)\right]
$$

where $L$ is equal to the determinant of $\boldsymbol{V}$. This summation extends over all integer vectors $\boldsymbol{k}$. If a bandlimited MD sig- 
nal has to be sampled without causing aliasing, we can do this by appropriate choice of $\boldsymbol{V}$ so as to ensure that no two terms in the preceding summation overlap for any $\omega$.

Digital processing of sampled multidimensional signals is discussed in [111]-[114]. An excellent overview on the sampling of multidimensional signals, including a review of lattices, can be found in [113].

The minimum sampling density required to avoid aliasing depends on the shape of the basic reciprocal lattice cell. By careful choice of $V$, we can minimize this density. In particular, notice that a diagonal $\boldsymbol{V}$ (i.e., rectangular sampling) does not necessarily lead to minimum density. This, in fact, is the motivation for the introduction of generalized nonrectangular sampling. For example, if $X_{a}(\Omega)$ is restricted to a circular region of support, then a particular choice of $V$ known as the "hexagonal sampling matrix," is known to result in smaller sampling density [109].

The generalized multidimensional decimator takes an input signal $x(n)$ and produces the output $y(n)=x(D n)$ where $D$ is a $M \times M$ nonsingular matrix of integers. Several standard multirate topics, such as the design of decimation and interpolation filters, design of filter-banks, and multistage decimator design techniques, have been recently extended to the MD case by several authors. For the case of separable systems, an interesting scheme to obtain perfect reconstruction with IIR filters (based on all-pass building blocks as in section VI) is reported in [115]. Reference [116] extends the perfect reconstruction results of [31] to the $2 \mathrm{D}$ rectangular decimation case. References [117] and [118] deal with some of these extensions for the case of generalized (nonrectangular) decimators and interpolators. Extension of the concept of polyphase representation to the (nonrectangular) MD case can be obtained by using the ideas of "cosets of lattices" [113]. In fact, it is shown in [118] that the perfect reconstruction techniques for maximally decimated analysis/synthesis systems reported in [31] can be generalized to the MD case simply by making use of such a generalized multidimensional polyphase decomposition.

\section{Xi. Open Problems and Concluding Remarks}

Even though the multirate filter bank area has reached a certain level of maturity, there do remain certain open questions and research problems of considerable interest.

First, consider the FIR two-channel perfect reconstruction system based on the Smith Barnwell approach. As we saw in section VI-B, the design issue is to find a spectral factor $H_{0}(z)$ of a zero-phase FIR half-band filter. Once $H_{0}(z)$ is found, the remaining filters are completely known (through the relations (56) and (58)). There are two ways to design $H_{0}(z)$. The first is to find a zero-phase half-band filter $H(z)$ of appropriate specifications and then find a spectral factor of $H(z)$ by a zero-finding approach or by cepstral methods [40]. The second procedure is to use the fact that the lattice of Fig. 28 automatically gives rise to $H_{0}(z)$ and $H_{1}(z)$ with appropriate properties as elaborated in section VI-C. The difficulty with the first approach is that if $A_{s}$ is the desired stopband attenuation of $H_{0}(z)$, then $H(z)$ has to have an attenuation of $2 A_{s} \mathrm{~dB}$. For large $A_{s}$, it is therefore difficult (and numerically inaccurate) to do the factorization. The second approach overcomes this problem because no spectral factorization is involved. However, it has the disadvantage of requiring long computer time for optimiza- tion of the lattice coefficients (and for this reason, a number of tables of filter-coefficients are published in [65] so that the designer does not have to do the optimization), even though perfect reconstruction is structurally guaranteed. The problem of efficient design of $H_{0}(z)$ (so that it takes only about as much time as a standard single-rate equiripple design [119] of comparable order) is still open. Stated in a completely different way (section VI-C), we wish to design a good low-pass filter $H_{0}(z)$ such that its two polyphase components are power complementary. What is the most efficient way to do this?

The second problem has to do with the all-pass breakup described in section VI-A. The conditions under which an IIR filter $H_{0}(z)$ can be expressed as a sum of two all-pass functions is well known [42], [43]; and digital Butterworth, Chebyshev, and Elliptic filters happen to satisfy this condition. Such transfer functions are useful in the design of two-channel QMF banks. For the M-band case, it would be necessary to express a transfer function as sum of $M$ all-pass functions (this also enables us to obtain a uniform DFT bank). Even though such designs have appeared in the literature [39], the problem of finding a set of necessary and sufficient conditions on $H_{0}(z)$ (analogous to [42, page 353]), that will permit such all-pass decomposition is open.

In section VII we saw that the pseudo-circulant property of $\boldsymbol{P}(z)$ is necessary and sufficient for alias-cancellation in QMF banks. Diagonal matrices of the form $P_{0}(z) I$ are clearly special cases of pseudo-circulants. However, all examples of alias-free QMF banks reported in the literature have $\boldsymbol{P}(z)$ $=P_{0}(z) I$ (or minor permutations of it), which is not a necessary condition. If we relax this condition, does it result in more efficient designs? Assuming that this is so, what is a systematic procedure to arrive at such designs? These are unresolved questions.

In section VI we saw that in order to design FIR perfect reconstruction QMF banks, it is really only necessary to force the determinant of $E(z)$ to be a delay. This was done by forcing $\boldsymbol{E}(z)$ to be lossless, primarily because losslessness is easier to force structurally, giving rise to useful design techniques (and, moreover, the synthesis filters are thereby guaranteed to have the same length as analysis filters, as seen from (74)). If we give up the lossless condition and keep $\boldsymbol{E}(z)$ as general as possible (subject of course to the determinant condition), then we can get more general (perhaps more efficient) designs. In the two-channel case, for example, in order to get FIR perfect reconstruction QMF banks with good analysis filters which are at the same time linearphase, it is necessary to give up losslessness [67].

An obvious way to force the determinant of $\boldsymbol{E}(z)$ to be a delay is to express $E(z)$ as

$$
\boldsymbol{E}(z)=\boldsymbol{R}_{K} \mathbf{\Lambda}_{K}(z) \boldsymbol{R}_{K-1} \boldsymbol{\Lambda}_{K-1}(z) \cdots \boldsymbol{R}_{1} \mathbf{\Lambda}_{1}(z) \boldsymbol{R}_{0}
$$

where $\boldsymbol{R}_{n}$ are $M \times M$ constant nonsingular matrices and $\Lambda_{n}(z)$ are $M \times M$ diagonal matrices with delay elements on the diagonals. Note that $(60)-(62)$ represent a special case of this with $M=2$. Now the preceding product form does not cover every possible $M \times M E(z)$ whose determinant is a delay. Here is the open problem: find a structure (or a mathematical expression) such that it represents every possible $\boldsymbol{E}(z)$ of a given degree with determinant equal to a delay, and which does not represent any $E(z)$ whose determinant is not a delay. If such a structure can be found, its parameters can then be optimized on a computer to obtain good 
analysis filters as done in section VIII. Even though this problem has not been formulated or solved to this degree of generality, special cases of this can be found in [67] and [87] (to obtain linear-phase FIR PR QMF banks) and in [55] (for obtaining special nonconstant passband magnitude responses for analysis filters in FIR PR QMF banks). One approach towards a generalized solution is the following observation: an $M \times M$ polynomial matrix $\boldsymbol{E}(z)$ in the variable $z^{-1}$ has determinant equal to a delay if and only if it can be expressed in the form

$$
\boldsymbol{E}(z)=\boldsymbol{U}(z) \mathbf{\Lambda}(z) \boldsymbol{V}(z)
$$

where $\boldsymbol{\Lambda}(z)$ is a diagonal matrix of delays (with nondecreasing powers as we go down the diagonal) and $\boldsymbol{U}(z)$ and $\boldsymbol{V}(z)$ are polynomial matrices in $z^{-1}$ with determinants equal to nonzero constants (in other words, $\boldsymbol{U}(z)$ and $V(z)$ are "unimodular" matrices [120])). This observation follows from the Smith-McMillan form of decomposition for polynomial matrices [120, Ch. 6], [121, Ch. 6]. The preceding problem then reduces essentially to one of characterizing causal unimodular matrices: one seeks to develop a structure whose multiplier parameters span all unimodular matrices; and conversely every transfer matrix generated by the structure is required to be causal and unimodular. Certain general factorization theorems such as those in [122] may give a starting point for this problem.

Fig. 48 helps to visualize the FIR perfect reconstruction family. The largest set in this Venn diagram is the set of all

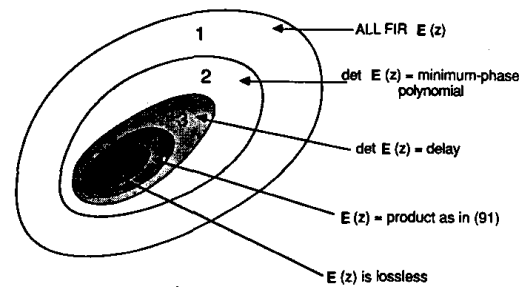

Fig. 48. Venn diagram, summarizing families of FIR QMF banks for perfect reconstruction.

FIR analysis banks. We then have the subset for which the determinant of $\boldsymbol{E}(z)$ is a minimum-phase polynomial so that a stable synthesis bank (not necessarily FIR) for perfect reconstruction exists. The next subset is such that det $\boldsymbol{E}(z)$ is actually a delay so that an FIR synthesis bank for perfect reconstruction exists. The synthesis filters can, however, be longer than the analysis filters. The fourth subset is such that $\boldsymbol{E}(z)$ can be expressed as in (91) (which obviously guarantees that the determinant is a delay). The synthesis filters for perfect reconstruction, in principle, can again be longer than the analysis filters. The last and the smallest subset is such that $\boldsymbol{E}(z)$ is lossless. In this case the synthesis filters have the same length as the analysis filters, and moreover are given by the closed form formula (74). The advantage of subset \#5 is that it gives rise to lattice structures, which in turn lead to simple design procedures for the analysis filters, ensuring that the filter-responses are nicely bounded. The disadvantage is that, in the two-channel case, losslessness is not compatible with the linear-phase requirement of analysis filters (unless they are trivial, as in (37)). In [67], losslessness has been relaxed to obtain linearphase two-channel QMF banks with perfect reconstruc- tion. There are two cases considered at length in [67]. These belong to subsets \#4 and \#3, respectively.

Another open area in multirate DSP is the design of QMF banks with nonuniform decimation ratio. This means that the decimators in Fig. 29(a) are not all equal to $M$ but depend on the subband. For example, let $n_{k}$ represent the decimation ratio for the $k$ th subband with $0 \leq k \leq M-1$ (the passband widths of the filters are, of course, accordingly adjusted to be nonuniform). These integers should clearly satisfy $\Sigma 1 / n_{k}=1$, to preserve the average number of samples per unit time. Examples of such systems have been generated in the past by combining smaller QMF bank modules in some way (such as in a tree structure [4]). Some of the unresolved issues in the more general case are how to obtain alias cancellation (and, for the more ambitious designer, perfect reconstruction) with FIR filters. What are the necessary and sufficient conditions for these, and how can these be forced structurally? Does losslessness still have a role? Some progress in this direction has been reported in [123].

Finally, there has been some progress in the design of two-dimensional filter banks, as mentioned in section $\mathrm{X}$. However, systematic procedures for obtaining completely general lattice structures for lossless polyphase matrices are yet to be developed, due to lack of neat factorization theorems in the general case. Factorization theorems for restricted special cases can be found in [82]. Finally, the 2D versions of practically all the $1 \mathrm{D}$ open problems are open as well.

\section{Nomenclature}

$\begin{array}{ll}A C & \text { All-pass complementary. } \\ A / D & \text { Analog to digital. } \\ A D P C M & \text { Adaptive delta pulse code modulation. } \\ A L D & \text { Aliasing distortion. } \\ A M D & \text { Amplitude distortion. } \\ A P C M & \text { Adaptive pulse code modulation. } \\ A P U & \text { Additions per unit time. } \\ D / A & \text { Digital to analog. } \\ D C & \text { Doubly complementary. } \\ D F T & \text { Discrete Fourier transform. } \\ D S P & \text { Digital signal processing. } \\ E C & \text { Euclidean complementary. } \\ F D M & \text { Frequency division multiplexing. } \\ F I R & \text { Finite-length impulse response. } \\ G P P & \text { Generalized polyphase. } \\ I D F T & \text { Inverse discrete Fourier transform. } \\ I F I R & \text { Interpolated finite-duration impulse response. } \\ I I R & \text { Infinite-duration impulse response. } \\ L B R & \text { Lossless bounded real. } \\ L O T & \text { Lapped orthogonal transform. } \\ L P T V & \text { Linear periodically time-varying. } \\ L T I & \text { Linear time-invariant. } \\ L T V & \text { Linear time-varying. } \\ M D & \text { Multidimensional. } \\ M O S & \text { Mean opinion score. } \\ M P U & \text { Multiplications per unit-time. } \\ P C & \text { Power complementary. } \\ P H D & \text { Phase distortion. } \\ P R & \text { Perfect reconstruction. } \\ Q M F & \text { Quadrature mirror filter. } \\ T D M & \text { Time-domain multiplexing. } \\ 2 D & \text { Two-dimensional. } \\ & \end{array}$




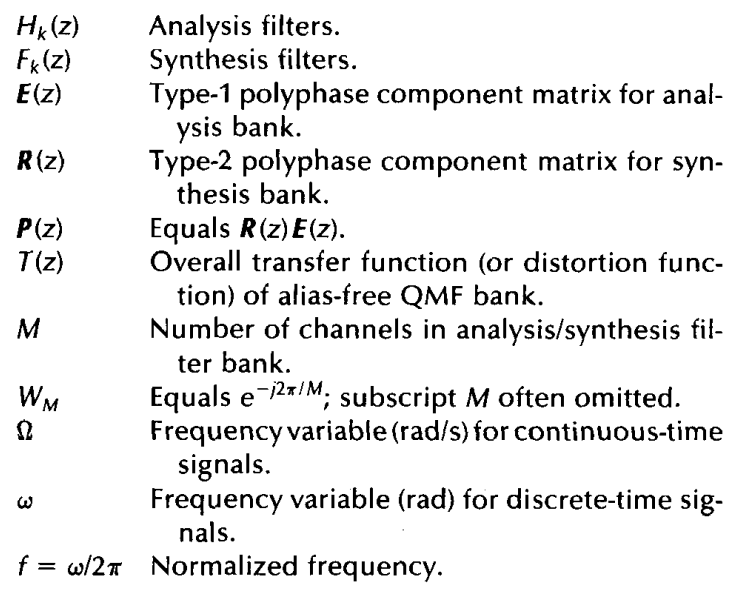

APPENDIX A

CONDITION FOR Equivalence OF Figs. 6(a) AND 6(b)

By applying the relations (3a), (3b) to Figs. 6(a) and 6(b) we see that

$$
\gamma_{1}(z)=\frac{1}{M} \sum_{k=0}^{M-1} X\left(z^{L / M} W^{k L}\right)
$$

and

$$
Y_{2}(z)=\frac{1}{M} \sum_{k=0}^{M-1} X\left(z^{L / M} W^{k}\right)
$$

The summations (A.1) and (A.2) are identical except for the powers of $W$ appearing in each. The right sides of (A.1) and (A.2) are identical if and only if the collection of $M$ numbers $W^{k L}, 0 \leq k \leq M-1$ is equal to the collection $W^{k}, 0 \leq k$ $\leq M-1$.

We know that there are precisely $M$ possible values for $W^{n}$ when $n$ runs through the set $0 \leq n \leq M-1$. Since $W^{k}$ $\neq W^{n}$ for $0 \leq k<n \leq M-1$, all the $M$ distinct values of the powers of $W$ are present in the set $W^{k}, 0 \leq k \leq M-$ 1. The expression (A.1) is therefore identical to (A.2) if and only if $W^{k L} \neq W^{n L}$ for any $k, n$ such that $0 \leq k<n \leq M$ -1 (i.e., if and only if $(k-n) L \neq p M$ for an integer $p$ ). This in turn is true if and only if $M$ and $L$ are relatively prime (i.e., do not share a common factor greater than unity).

APPENDIX B

Relation Between $M$ th Band Filters and Power-

Complementary Filters

Let $H(z)$ be any transfer function expressed in Type-1 polyphase form (10). Define

$$
H_{n}(z) \triangleq H\left(z W^{-n}\right)=\sum_{k=0}^{M-1} z^{-k} W^{k n} E_{k}\left(z^{M}\right)
$$

for $0 \leq n \leq M-1$, where $W=\mathrm{e}^{-j 2 \pi / M}$. This set of $M$ transfer functions can be represented in vector form as

$$
\left[\begin{array}{c}
H_{0}(z) \\
H_{1}(z) \\
\vdots \\
H_{M-1}(z)
\end{array}\right]=\mathbf{w}\left[\begin{array}{cccc}
1 & 0 & \ldots & 0 \\
0 & z^{-1} & \ldots & 0 \\
\vdots & \vdots & \ddots & \vdots \\
0 & 0 & \ldots & z^{-(M-1)}
\end{array}\right]\left[\begin{array}{c}
E_{0}\left(z^{M}\right) \\
E_{1}\left(z^{M}\right) \\
\vdots \\
E_{M-1}\left(z^{M}\right)
\end{array}\right]
$$

where $\boldsymbol{W}$ is the $M \times M D F T$ matrix [7], [8]. Since $\boldsymbol{W}$ is unitary, it is (trivially) paraunitary (section VI-C). If the set $\left[E_{0}(z), E_{1}(z)\right.$, $\left.\cdots, E_{M-1}(z)\right]$ is power complementary (i.e., if the column vector $\left[E_{0}(z), E_{1}(z), \cdots, E_{M-1}(z)\right]^{T}$ is paraunitary), then the $M \times 1$ matrix on the LHS of (A.4) is paraunitary (because it is a product of paraunitary matrices). This, in turn, means that the set $H_{k}(z), 0 \leq k \leq M-1$ is power complementary. In other words, if we define $G(z) \triangleq \tilde{H}(z) H(z)$, then $G(z)$ satisfies $\Sigma_{k=0}^{M-1} G\left(z W^{-k}\right)=$ constant, so that it is an $M$ th band filter.

Conversely, assuming that $G(z)$ is an $M$ th band filter, we can prove that the set of polyphase components $\left[E_{0}(z), E_{1}(z)\right.$, $\left.\cdots, E_{M-1}(z)\right]$ is power complementary simply by inverting the two $M \times M$ matrices in (A.4) and carrying out a similar argument as in the preceding.

\section{ACKNOWLEDGMENT}

I would like to thank Dr. R. E. Crochiere of the AT\&T Bell Labs for his encouragement in the preparation of this article. It is a privilege to acknowledge the useful discussions I have had with Dr. M. Bellanger of TRT, France, Prof. M. Vetterli of Columbia University, Profs. Smith and Barnwell of Georgia Institute of Technology, and Prof. T. Ramstad of the Norwegian Institute of Technology. Also, the input from reviewers resulted in useful improvements of this paper. Finally, the valuable "multirate interaction" I have had with my graduate students during the last few years is gratefully acknowledged. In particular, Zinnur Doğanata's interactions on lossless systems, Vincent Liu's interactions on nonuniform sampling and on 2D systems, and Truong Nguyen's interactions on symmetric and linear-phase QMF banks have been particularly beneficial.

\section{RefERENCES}

[1] R. W. Schafer and L. R. Rabiner, "A digital signal processing approach to interpolation," Proc. IEEE, vol. 61, pp. 692-702, June 1973.

[2] G. Oetken, T. W. Parks, and H. W. Schussler, "New results in the design of digital interpolators," IEEE Trans. Acoust. Speech Signal Proc., vol. 23, pp. 301-309, June 1975.

[3] R. E. Crochiere and L. R. Rabiner, "Interpolation and decimation of digital signals: A tutorial review," Proc. IEEE, vol. 69, pp. 300-331, Mar. 1981.

[4] R. E. Crochiere and L. R. Rabiner, Multirate Digital Signal Processing. Englewood Cliffs, NJ: Prentice Hall, 1983.

[5] P. P. Vaidyanathan, "A tutorial on multirate digital filter banks," in Proc. IEEE Int. Symp. on Circuits and Systems, Espoo, Finland, June 1988, pp. 2241-2248.

[6] M. R. Portnoff, "Time-frequency representation of digital signals and systems based on short-time Fourier analysis," IEEE Trans. Acoust. Speech Signal Proc., vol. 28, pp. 55-69, Feb. 1980.

[7] A. V. Oppenheim and R. W. Schafer, Digital Signal Processing. Englewood Cliffs, N): Prentice Hall, 1975.

[8] A. V. Oppenheim, A. S. Willsky, and I. T. Young, Signals and Systems. Englewood Cliffs, NJ: Prentice Hall, 1983.

[9] A. Antoniou, Digital Filters: Analysis and Design. New York: McGraw Hill, 1979.

[10] R. Ansari and Bede Liu, "Interpolators and decimators as periodically time varying filters," in Proc. IEEE Int. Symp. Circuits and Systems, 1981, pp. 447-450.

[11] M. Bellanger, G. Bonnerot, and M. Coudreuse, "Digital filtering by polyphase network: Application to sample rate alteration and filter banks," IEEE Trans. Acoust. Speech Signal Proc., vol. 24, pp. 109-114, Apr. 1976.

[12] K. Swaminathan and P. P. Vaidyanathan, "Theory and design of uniform DFT, parallel, quadrature mirror filter banks," IEEE Trans. Circuits Syst., vol. 33, pp. 1170-1191, Dec. 1986. 
[13] $H$. Scheuermann and $H$. Gockler, "A comprehensive survey of digital transmultiplexing methods," Proc. IEEE, vol. 69, pp. 1419-1450, Nov. 1981.

[14] M. Vetterli, "A theory of multirate filter banks," IEEE Trans. Acoust. Speech Signal Proc., vol. 35, pp. 356-372, Mar. 1987.

[15] Special Issue on Digital Audio, IEEE ASSP Mag., vol. 2, Oct. 1985.

[16] P. P. Vaidyanathan, "Design and implementation of digital FIR filters," in Handbook on Digital Signal Processing, D. F. Elliott, Ed. New York: Academic, 1987, pp. 55-172.

[17] L. R. Rabiner and B. Gold, Theory and Application of Digital Signal Processing. Englewood Cliffs, NJ: Prentice Hall, 1975.

[18] R. E. Crochiere, "On the design of sub-band coders for low bit rate speech communication," Bell Syst. Tech. J., pp. 747771, May-June 1977.

[19] R. E. Crochiere, "Sub-band coding," Bell Syst. Tech. J., pp. 1633-1654, Sept. 1981.

[20] N. S. Jayant and P. Noll, Digital Coding of Waveforms. Englewood Cliffs, NJ: Prentice Hall, 1984.

[21] C. Galand and D. Esteban, "16 Kbps real-time QMF sub-band coding implementation," in Proc. Int. Conf. on Acoust. Speech and Signal Proc., Denver, CO, Apr. 1980, pp. 332335.

[22] R. J. Safranek, K. MacKay, N. S. Jayant, and T. Kim, “Image coding based on selective quantization of the reconstruction noise in the dominant sub-band," in Proc. IEEE Int. Conf. on ASSP, Apr. 1988, pp. 765-768.

[23] M. J. T. Smith and S. L. Eddins, "Subband coding of images with octave band tree structures," in Proc. IEEE Int. Conf. on ASSP, Dallas, TX, Apr. 1987, pp. 1382-1385.

[24] J. W. Woods and S. D. O'Neil, "Subband coding images," IEEE Trans. Acoust. Speech Signal Proc., vol. 34, pp. 12781288 , Oct. 1986.

[25] M.Vetterli, "Multi-dimensional sub-band coding: some theory and algorithms," Signal Proc., vol. 6, pp. 97-112, Apr. 1984.

[26] H. Gharavi and A. Tabatabai, "Sub-band coding of digital images using two dimensional quad rature mirror filtering," in Proc. SPIE Conf. on Visual Comm. and Image Proc., vol. 707, Cambridge, MA, Sept. 1986, pp. 51-61.

[27] H. Gharavi and A. Tabatabai, "Sub-band coding of monochrome and color images," IEEE Trans. Circuits Syst., vol. 35. pp. 207-214, Feb. 1988.

[28] R. M. Gray, "Vector quantization," IEEE ASSP Mag., voi. 1, pp. 4-29, Apr. 1984.

[29] R. V. Cox, D. E. Bock, K. B. Bauer, I. D. Johnston, and I. H. Snyder, "The analog voice privacy system," AT\&T Tech. J., vol. 66, pp. 119-131, Jan-Feb. 1987

[30] P. P. Vaidyanathan and Vincent C. Liu, "Classical sampling theorems in the context of multirate and polyphase digital filter bank structures," IEEE Trans. Acoust. Speech Signal Proc., vol. 36, pp. 1480-1495, Sept. 1988.

[31] P. P. Vaidyanathan, "Theory and design of $M$-channel maximally decimated quadrature mirror filters with arbitrary $M$, having perfect reconstruction property," IEEE Trans. Acoust. Speech Signal Proc., vol. 35, pp. 476-492, Apr. 1987.

[32] C. C. Hsiao, "Polyphase filter matrix for rational sampling rate conversions," in Proc. IEEE Int. Conf. on ASSP, Dallas, TX, Apr. 1987, pp. 2173-2176.

[33] R. J. McEliece, The Theory of Information and Coding, New York: Addison-Wesley, 1977.

[34] T. Thong, "Practical consideration for a continuous time digital spectrum analyzer," in Proc. IEEE Int. Symp. on Circuits and Systems, Portland, OR, in May 1989, pp. 1047-1050.

[35] F. Mintzer, "On half-band, third-band and Nth band FIR filters and their design," IEEE Trans. Acoust. Speech Signal Proc., vol. 30, pp. 734-738, Oct. 1982.

[36] P.P. Vaidyanathan and T. Q. Nguyen, "A trick for the design of FIR half-band filters," IEEE Trans. Circuits Syst., vol. 34, pp. 297-300, Mar. 1987.

[37] K. H. Mueller, "A new approach to optimum pulse shaping in sampled systems using time-domain filtering," Bell Syst. Tech. J., vol. 52, pp. 723-729, May-June 1973.

[38] P. P. Vaidyanathan and T. Q. Nguyen, "Eigenfilters: A new approach to least-squares FIR filter design and applications including Nyquist filters," IEEE Trans. Circuits Syst., vol. 34, pp. 11-23, Jan. 1987.
[39] M. Renfors and T. Saramaki, "Recursive $N$ th band digital filters, Parts I and II," IEEE Trans. Circuits Syst., vol. 34, pp. 24-51, Jan. 1987.

[40] G. A. Mian and A. P. Nainer, "A fast procedure to design equiripple minimum-phase FIR filters," IEEE Trans. Circuits Syst., vol. 29, pp. 327-331, May 1982.

[41] B. Friedlander, "A lattice algorithm for factoring the spectrum of a moving average process," IEEE Trans. Automatic Cont., vol. 28, pp. 1051-1055, Nov. 1983.

[42] P. P. Vaidyanathan, S. K. Mitra, and Y. Neuvo, "A new approach to the realization of low sensitivity IIR digital filters," IEEE Trans. Acoust. Speech Signal Proc., vol. 34, pp. 350-361, Apr. 1986.

[43] P. P. Vaidyanathan, P. Regalia, and S. K. Mitra, "Design of doubly complementary IIR digital filters using a single complex allpass filter, with multirate applications," IEEE Trans. Circuits Syst., vol. 34, pp. 378-389 Apr. 1987.

[44] P. A. Regalia, N. Fujii, S. K. Mitra, and Y. Neuvo, "Active RC crossover networks with adjustable characteristics," I. Audio Engr. Soc., pp. 24-30, Jan-Feb. 1987.

[45] R. M. Bullock III, "A new three-way allpass crossover network design," J. Audio Engr. Soc., pp. 315-322, May 1986.

[46] A. Croisier, D. Esteban, and C. Galand "Perfect channel splitting by use of interpolation/decimation/tree decomposition techniques," presented at Int. Symp. on Info., Circuits, and Systems, Patras, Greece, 1976.

[47] C. R. Galand and H. J. Nussbaumer "New quadrature mirror filter structures," IEEE Trans. Acoust. Speech Signal Proc., vol. 32, pp. 522-531, June 1984.

[48] I. D. Johnston, "A filter family designed for use in quadrature mirror filter banks," in Proc. IEEE Int. Conf. ASSP, Apr. 1980, pp. 291-294.

[49] V. K. Jain and R. E. Crochiere, "Quadrature mirror filter design in the time domain," IEEE Trans. Acoust. Speech Signal Proc., vol. 32, pp. 353-361, Apr. 1984.

[50] P.P. Vaidyanathan, "Quadrature mirror filter banks, $M$-band extensions and perfect-reconstruction techniques," IEEE ASSP Mag., vol. 4, pp. 4-20, July 1987.

[51] L. Gaszi, "Explicit formulas for lattice wave digital filters," IEEE Trans. Circuits Syst., vol. 32, pp. 68-88, Jan. 1985.

[52] S. K. Mitra and K. Hirano, "Digital allpass networks," IEEE Trans. Circuits Syst., vol. 21, pp. 688-700, Sept. 1974.

[53] A. H. Gray, Jr., "Passive cascaded lattice digital filters," IEEE Trans. Circuits Syst., vol. 27, pp. 337-344, May 1980.

[54] M. J. T. Smith and T. P. Barnwell III, "Exact reconstruction techniques for tree-structured sub-band coders," IEEE Trans. ASSP, pp. 434-441, June 1986.

[55] E. Viscito and J. Allebach, "The design of tree-structured $M$ channel filter banks using perfect reconstruction filter blocks," in Proc. IEEE Int. Conf. on ASSP, New York, NY, Apr. 1988, pp. 1475-1478.

[56] N. Balabanian and T. A. Bickart, Electrical Network Theory. New York: John Wiley, 1969.

[57] B. D. O. Anderson and S. Vongpanitlerd, Network Analysis and Synthesis. New York: Prentice Hall, 1973.

[58] V. Belevitch, Classical Network Theory. San Francisco: Holden Day, 1968.

[59] A. Fettweis, “Digital filter structu res related to classical filter networks," in Digital Signal Processing /I. New York: IEEE ASSP Society, IEEE Press, 1975, pp. 475-485.

[60] P. P. Vaidyanathan and S. K. Mitra, "Low passband sensitivity digital filters: A generalized viewpoint and synthesis procedures," Proc. IEEE, vol. 72, pp. 404-423, Apr. 1984.

[61] P. P. Vaidyanathan and S. K. Mitra, "A unified structural interpretation of some well-known stability-test procedures for linear systems," Proc. IEEE, vol. 75, pp. 478-497, Apr. 1987.

[62] P. P. Vaidyanathan and Z. Doğanata, "The role of lossless systems in modern digital signal processing: A tutorial," Special Issue on Circuits and Systems, IEEE Trans. Education, August 1989 (to appear).

[63] P. P. Vaidyanathan, T. Q. Nguyen, Z. Doğanata, and T. Saramaki, "Improved approach for design of perfect reconstruction FIR QMF banks with lossless polyphase structures," IEEE Trans. Acoust. Speech Signal Proc., vol. 37, July 1989 (to appear).

[64] Z. Doğanata, P. P. Vaidyanathan, and T. Q. Nguyen, "General synthesis procedures for FIR lossless transfer matrices, 
for perfect-reconstruction multirate filter bank applications," IEEE Trans. Acoust. Speech Signal Proc., vol. 36, pp. 1561-1574, Oct. 1988.

[65] P. P. Vaidyanathan and P.-Q. Hoang "Lattice structures for optimal design and robust implementation of two-channel perfect reconstruction QMF banks," IEEE Trans. Acoust. Speech Signal Proc., vol. 36, pp. 81-94, Jan. 1988.

66] F. Mintzer, "Filters for distortion-free two-band multirate filter banks," IEEE Trans. Acoust. Speech Signal Proc., vol. 33, pp. 626-630, June 1985.

[67] T. Q. Nguyen and P. P. Vaidyanathan,'Two-channel perfect reconstruction FIR QMF structures which yield linear phase FIR analysis and synthesis filters," IEEE Trans. Acoust. Speech Signal Proc., vol. 37, pp. 676-690, May 1989.

[68] C. R. Galand and H. J. Nussbaumer, "Quadrature mirror filters with perfect reconstruction and reduced computational complexity," in Proc. IEEE Int. Conf. on ASSP, Apr. 1985, pp. 525-528.

[69] Z. Doğanata and P. P. Vaidyanathan, "On one-multiplier implementations of FIR lattice structures," IEEE Trans. Circuits Syst., vol. 34, pp. 1608-1609, Dec. 1987.

[70] P. P. Vaidyanathan and S. K. Mitra, "Polyphase networks, block digital filtering, LPTV systems, and alias-free QMF banks: A unified approach based on pseudocirculants," IEEE Trans. Acoust. Speech Signal Proc., vol. 36, pp. 381 391, Mar. 1988

[71] T. A. Ramstad "Analysis/synthesis filter banks with critical sampling," presented at Int. Conf. on Digital Signal Processing, Florence, Italy, Sept. 1984.

[72] M. J.T. Smith and T. P. Barnwell, III, "A new filter-bank theory for time-frequency representation," IEEE Trans. Acoust. Speech Signal Proc., vol. 35, pp. 314-327, Mar. 1987.

[73] C. S. Burrus, "Block implementation of digital filters," IEEE Trans. Circuit Theory, vol. 18, pp. 697-701, Nov. 1971.

[74] S. K. Mitra and R. Gnanasekaran, "Block implementation of recursive digital filters: New structures and properties," IEEE Trans. Circuits Syst., vol. 25, pp. 200-207, Apr. 1978.

[75] C. W. Barnes and S. Shinnaka, "Block-shift invariance and block implementation of discrete-time filters," IEEE Trans. Circuits Syst., vol. 27, pp. 667-672, Aug. 1980.

[76] P. J. Davis, Circulant Matrices. New York: Wiley, 1979.

[77] T. G. Marshall, Jr., "Structures for digital filter banks," in Proc. IEEE Int. Conf. Acoust. Speech Signal Proc., Paris, Apr. 1982, pp. 315-318.

[78] M. Vetterli, "Running FIR and IIR filtering using multirate filter banks," IEEE Trans. Acoust. Speech Signal Proc., vol. 36, pp. 730-738, May 1988.

[79] Z. J. Mou and P. Duhamel, "Fast FIR filtering: Algorithms and implementations," Signal Proc., vol. 13, pp. 377-384, Dec. 1987.

[80] M. Vetterli, "Filter banks allowing for perfect reconstruction," Signal Proc., vol. 10, pp. 219-244, Apr. 1986.

[81] P.P.Vaidyanathan, "The discrete-time bounded-real lemma in digital filtering," IEEE Trans. Circuits Syst., vol. 32, pp. 918924, Sept. 1985.

[82] V. C. Liu and P. P. Vaidyanathan, "On factorization of 2D digital FIR lossless matrices for 2D QMF bank applications," IEEE Trans. Circuits Syst., to appear, 1990.

[83] P.P. Vaidyanathan, "Passive cascaded lattice structures for low sensitivity FIR filter design, with applications to filter banks," IEEE Trans. Circuits Syst., vol. 33, pp. 1045-1064, Nov. 1986.

[84] F. D. Murnaghan, The Unitary and Rotation Groups. Washington, D.C.: Spartan Books, 1962.

[85] Z. Doğanata, and P. P. Vaidyanathan, "Minimal structures for the implementation of digital rational lossless systems," IEEE Trans. Acoust. Speech and Signal Processing, to appear, 1990.

[86] The IMSL Library: A set of Fortran subroutines for mathematics and statistics.

[87] T. Q. Nguyen and P. P. Vaidyanathan, "Design techniques for M-channel perfect reconstruction FIR QMF structures which yield linear-phase maximally free analysis filters," IEEE Trans. Acoustics Speech Signal Proc., to appear.

[88] J. H. Rothweiler, "Polyphase quadrature filters, a new subband coding technique," in Proc. IEEE Int. Conf. on ASSP, Boston, MA, Apr. 1983, pp. 1980-1983.
[89] P. L. Chu, "Quadrature mirror filter design for an arbitrary number of equal bandwidth channels," IEEE Trans. Acoust. Speech Signal Proc., vol. 33, pp. 203-218, Feb. 1985.

[90] T. Q. Nguyen and P. P. Vaidyanathan, "Maximally decimated perfect-reconstruction FIR filter banks with pairwise mirror-image analysis (and synthesis) frequency responses," IEEE Trans. Acoust. Speech Signal Proc., vol. 36, pp. 693-706, May 1988.

[91] H. S. Malvar and D. H. Staelin, "Reduction of blocking effects in image coding with a lapped orthogonal transform," in Proc. IEEE Int. Conf. on ASSP, New York, Apr. 1988, pp. 781-784.

[92] M. Vetterli and D. Le Gall, "Perfect reconstruction FIR filter banks: Some properties and factorizations," IEEE Trans. Acoustics Speech Signal Proc., to appear, 1990.

[93] J. P. Princen and A. B. Bradley, "Analysis/synthesis filter bank design based on time domain aliasing cancellation," IEEE Trans. Acoustics Speech Signal Proc., vol. 23, pp. 11531161, Oct. 1986

[94] C. E. Shannon, "Communications in the presence of noise," Proc. IRE, vol. 37, pp. 10-21, Jan. 1949.

[95] J. M. Whittaker, "The Fourier theory of the cardinal functions," Proc. Math. Soc. Edinburgh, vol. 1, pp. 169-176, 1929.

[96] A. J. Jerri, "The Shannon sampling theorem-lts various extensions and applications: A tutorial review," Proc. IEEE, pp. 1565-1596, Nov. 1977.

[97] H. S. Black, Modulation Theory. New York: Van Nostrand, 1953.

[98] J. L. Brown, Jr., "Multi-channel sampling of lowpass signals," IEEE Trans. Circuits Syst., vol. 28, pp. 101-106, Feb. 1981.

[99] A. Papoulis, "Generalized sampling expansion," IEEE Trans. Circuits Syst., pp. 652-654, Nov. 1977.

[100] P. P. Vaidyanathan and V. C. Liu, "Compression of twodimensional band-limited signals using sub-sampling theorems," J. Institution Electron. Telecomm. Eng., India, vol. 34, no. 5, pp. 416-422, 1988.

[101] V.C. Liu and P. P. Vaidyanathan, "Efficient reconstruction of bandlimited signals from nonuniformly decimated versions by use of polyphase filter banks," IEEE Trans. Acoust. Speech and Signal Processing, Nov. 1990, to appear.

[102] Y. Neuvo, C.-Y. Dong, and S. K. Mitra, "Interpolated finite impulse response filters," IEEE Trans. Acoust. Speech Signal Proc., vol. 32, pp. 563-570, June 1984.

[103] Y. C. Lim, "Frequency response masking approach for the synthesis of sharp linear phase digital filters," IEEE Trans. Circuits Syst., vol. 33, pp. 357-364, Apr. 1986.

[104] S. Haykin, Adaptive Filter Theory. Englewood Cliffs, NJ: Prentice Hall, 1986

[105] B. Widrow and S. D. Stearns, Adaptive Signal Processing. Englewood Cliffs, NI: Prentice Hall, 1985.

[106] R. Bitmead and B. D. O. Anderson, "Adaptive frequency sampling filters," IEEE Trans. Acoust. Speech Signal Proc. vol. 29, pp. 684-693, June 1981.

[107] M. Dentino, J. McCool, and B. Widrow, “Adaptive filtering in the frequency domain," Proc. IEEE, vol, 66, pp. 1658-1659, Dec. 1978.

[108] A Gilloire and M. Vetterli, "Adaptive filtering in subbands," in Proc. IEEE Int. Conf. on ASSP, New York, Apr. 1988, pp. 1572-1575.

[109] D. E. Dudgeon and R. M. Mersereau, "Multidimensiona digital signal processing," Englewood Cliffs, NJ: Prentice Hall, 1984.

[110] D. P. Petersen and D. Middleton, "Sampling and reconstruction of wave-number limited functions in $\mathrm{N}$-dimensional Euclidean spaces," Inform. Control, vol. 5, pp. 279 323, 1962.

[111] R. M. Mersereau, "The processing of hexagonally sampled two-dimensional signals," Proc. IEEE, vol. 67, pp. 930-949, June 1979.

[112] G. Wackersreuther, "On two-dimensional polyphase filte banks," IEEE Trans. Acoust. Speech Signal Proc., vol. 34, pp. 192-199, Feb. 1986.

[113] E. Dubois, "The sampling and reconstruction of time-vary ing imagery with application in video systems," Proc. IEEE, vol. 73, pp. 502-522, Apr. 1985.

[114] R. M. Mersereau and T. C. Speake, "The processing of 
periodically sampled multidimensional signals" "IEEE Trans. Acoust. Speech Signal Proc., vol. 31, pp. 188-194, Feb. 1983.

[115] T. A. Ramstad, "IIR filterbank for subband coding of images," in Proc. IEEE Int. Symp. on Circuits and Systems, Espoo, Finland, June 1988, pp. 827-830.

[116] P.P. Vaidyanathan, "Perfect reconstruction QMF banks for two-dimensional applications," IEEE Trans. Circuits Syst., vol. 34, pp. 976-978, Aug. 1987.

[117] R. Ansari and S. H. Lee, "Two-dimensional multirate processing on nonrectangular grids: Theory and filtering procedures," preprint copy.

[118] E. Viscito and J. Allebach, "Design of perfect reconstruction multi-dimensional filter banks using cascaded Smith form matrices," in Proc. IEEE Int. Symp. on Circuits and Syst., Espoo, Finland, pp. 831-834, June 1988.

[119] J. H. McClellan and T. W. Parks, "A unified approach to the design of optimum FIR linear-phase digital filters," IEEE Trans. Circuit Theory, vol. 20, pp. 697-701, Nov. 1973.

[120] F. R. Gantmacher, The Theory of Matrices, vols. 1 and 2. New York: Chelsea Publishing, 1959.

[121] T. Kailath, Linear Systems. Englewood Cliffs, N]: Prentice Hall, 1980 .

[122] S. Tan and J. Vandewalle, "Fundamental factorization theorems for rational matrices over complex or real fields," in Proc. IEEE Int. Symp. on Circuits and Syst., Espoo, Finland, June 1988, pp. 1183-1186.

[123] P.-Q. Hoang and P. P. Vaidyanathan "Non-uniform multirate filter banks: Theory and design," in Proc. IEEE Int. Symp. on Circuits and Syst., Portland, OR, May 1989, pp. 371-374.

[124] A. G. Constantinides and R. A. Valenzuela, "A class of efficient interpolators and decimators with applications in transmultiplexers," in Proc. IEEE Int. Symp. on Circuits and Syst., Rome, May 1982, pp. 260-263.
[125] T. P. Barnwell III, "Sub-band coder design incorporating recursive quadrature filters and optimum ADPCM coders," IEEE Trans. Acoust. Speech Signal Proc., vol. 30, pp. 751-765, Oct. 1982.

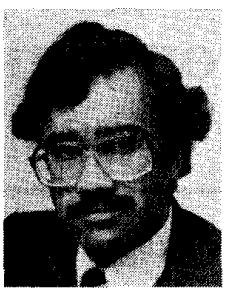

P. P. Vaidyanathan (Senior Member, IEEE) was born in Calcutta, India, on October 16 1954. He received the B.Sc. (Hons.) degree in physics and the $B$. Tech and $M$. Tech degrees in radiophysics and electronics, all from the University of Calcutta, India, in 1974, 1977, and 1979, respectively. He received the Ph.D. degree in electrical and computer engineering from the University of California, Santa Barbara, in 1982

He was a postdoctoral fellow at the University of California, Santa Barbara, from September 1982 to February 1983. In March 1983 he joined the California Institute of Technology, Pasadena, as an Assistant Professor of electrical engineering, and is currently as Associate Professor in the same department. His main research interests are in digital signal processing, multirate filter bank systems, filter design, adaptive filtering, and multivariable system theory.

Dr. Vaidyanathan served as Vice-Chairman of the Technical Program Committee for the 1983 IEEF International Symposium on Circuits and Systems and as an Associate Editor for the IEEE TRANSACTIONS ON CIRCUITS AND SYSTEMS for the period 1985-1987. He was a recipient of the Award for Excellence in Teaching at the California Institute of Technology for the year 1983-1984. He was also a recipient of the NSF's Presidential Young Investigator Award in 1986. In 1989 he received the IEEE ASSP Senior Award for his paper on multirate perfect-reconstruction filter banks. 